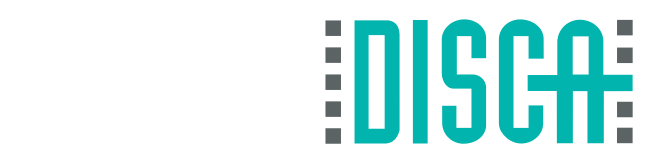

Departamento DE Informática DE Sistemas Y COMPUTADORES

\title{
Improving Message Dissemination in Opportunistic Networks
}

Thesis submitted in partial fulfillment of the requirements for the degree of Doctor of Philosophy in Computer Science.

By

Jorge Sergio Herrera Tapia

Advisors:

Dr. Pietro Manzoni

Dr. Enrique Hernández Orallo

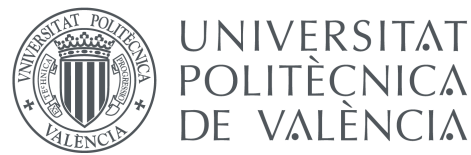

Universitat Politècnica de València

Valencia, Spain

June, 2017 

To my Family, in special to My Parents. 

Gaudeamus igitur, iuvenes dum sumus.

Anonymous. 



\section{Acknowledgements}

- Y eternal gratitude to my advisors Dr. Pietro Manzoni and Dr. Enrique W Hernández Orallo, for their noble, generous and invaluable scientific support in this research process, without their help this thesis would not have been finished. Also, I want to thanks to Drs. Carlos Calafate, Juan-Carlos Cano, and Andrés Tomás for their academic advice. Thanks you all Professors for your understanding and meticulous work in this research field, for your friendship and help in the good and bad moments, and for giving me the opportunity to study in the GRC research group, at "Universitat Politècnica de València".

Special thanks to Dr. Anna Foerster, and colleagues from the Comnets research group at the University of Bremen, who welcomed me in their research group during my internship, and provided a very friendly atmosphere.

Thanks to my family for their constant support, and for giving me that strength and optimism during this stage of studies, that was very demanding in all its dimensions.

Thanks to Ecuadorian friends of the GRC specially Jorge's, Óscar, and friends from other nationalities whom we shared a laboratory of effort and joys.

Finally, thanks to the Ecuadorian State through the "Secretaria Nacional de Educación Superior, Ciencia y Tecnología SENESCYT" and the "Universidad Laica Eloy Alfaro de Manabí ULEAM" for its supporting programs.

Thank you all.

Jorge Herrera Tapia Valencia, Spain June 15, 2017 



\section{Abstract}

D Ata transmission has become a need in various fields, like in social networks with the diverse interaction applications, or in the scientific and engineering areas where for example the use of sensors to capture data is growing, or in emergency situations where there is the imperative need to have a communication system to coordinate rescue operations. Wireless networks have been able to solve these issues to a great extent, but what can we do when a fixed supporting infrastructure is not available or becomes inoperative because of saturation? Opportunistic wireless networks are an alternative to consider in these situations, since their operation does not depend on the existence of a telecommunications infrastructure but they provide connectivity through the organized cooperation of users.

This research thesis focuses on these types of networks and is aimed at improving the dissemination of information in opportunistic networks analyzing the main causes that influence the performance of data transmission. Opportunistic networks do not depend on a fixed topology but depend on the number and mobility of users, the type and quantity of information generated and sent, as well as the physical characteristics of the mobile devices that users have to transmit the data. The combination of these elements impacts on the duration of the contact time between mobile users, directly affecting the information delivery probability.

This thesis starts by presenting a thorough "state of the art" study where we present the most important contributions related to this area and the solutions offered for the evaluation of the opportunistic networks, such as simulation models, routing protocols, simulation tools, among others. After offering this broad background, we evaluate the consumption of the resources of the mobile devices that affect the performance of the the applications of opportunistic networks, both from the energetic and the memory point of view.

Next, we analyze the performance of opportunistic networks considering either pedestrian and vehicular environments. The studied approaches include the use 
of additional fixed nodes and different data transmission technologies, to improve the duration of the contact between mobile devices.

Finally, we propose a diffusion scheme to improve the performance of data transmission based on extending the duration of the contact time and the likelihood that users will collaborate in this process. This approach is complemented by the efficient management of the resources of the mobile devices. 


\section{Resumen}

I A transmisión de datos se ha convertido en una necesidad en diversos ámbi$\perp$ tos, como en las redes sociales con sus diversas aplicaciones, o en las áreas científicas y de ingeniería donde, por ejemplo, el uso de sensores para capturar datos está creciendo, o en situaciones de emergencia donde impera la necesidad de tener un sistema de comunicación para coordinar las operaciones de rescate. Las redes inalámbricas actuales han sido capaces de resolver estos problemas en gran medida, pero ¿qué podemos hacer cuando una infraestructura de soporte fija no está disponible o estas se vuelven inoperantes debido a la saturación de peticiones de red? Las redes inalámbricas oportunísticas son una alternativa a considerar en estas situaciones, ya que su funcionamiento no depende de la existencia de una infraestructura de telecomunicaciones sino que la conectividad es a través de la cooperación organizada de los usuarios.

Esta tesis de investigación se centra en estos tipos de redes oportunísticas y tiene como objetivo mejorar la difusión de información analizando las principales causas que influyen en el rendimiento de la transmisión de datos. Las redes oportunísticas no dependen de una topología fija, sino que dependen del número y la movilidad de los usuarios, del tipo y cantidad de información generada y enviada, así como de las características físicas de los dispositivos móviles que los usuarios tienen para transmitir los datos. La combinación de estos elementos influye en la duración del tiempo de contacto entre usuarios móviles, afectando directamente a la probabilidad de entrega de información.

Esta tesis comienza presentando un exhaustivo estudio del "estado del arte", donde presentamos las contribuciones más importantes relacionadas con esta área y las soluciones existentes para la evaluación de las redes oportunísticas, tales como modelos de simulación, protocolos de enrutamiento, herramientas de simulación, entre otros. Tras ofrecer esta amplia compilación de investigaciones, se evalúa el consumo de recursos de los dispositivos móviles que afectan al rendimiento de las aplicaciones de redes oportunísticas, desde el punto de vista energético así como 
de la memoria.

A continuación, analizamos el rendimiento de las redes oportunísticas considerando tanto los entornos peatonales como vehiculares. Los enfoques estudiados incluyen el uso de nodos fijos adicionales y diferentes tecnologías de transmisión de datos, para mejorar la duración del contacto entre dispositivos móviles.

Finalmente, proponemos un esquema de difusión para mejorar el rendimiento de la transmisión de datos basado en la extensión de la duración del tiempo de contacto, y de la probabilidad de que los usuarios colaboren en este proceso. Este enfoque se complementa con la gestión eficiente de los recursos de los dispositivos móviles. 


\section{Resum}

A transmissió de dades s'ha convertit en una necessitat en diversos àmbits, com
ara en les xarxes socials amb les diverses aplicacions d'interacció, o en les àrees
científiques i d'enginyeria, en les quals, per exemple, l'ús de sensors per a capturar
dades creix en l'actualitat, o en situacions d'emergència en què impera la necessitat
de tenir un sistema de comunicació per a coordinar les operacions de rescat. Les
xarxes sense fil han sigut capaces de resoldre aquests problemes en gran manera,
però què podem fer quan una infraestructura de suport fixa no està disponible, o
bé aquestes es tornen inoperants a causa de la saturació de peticions de xarxa?
Les xarxes sense fil oportunistes són una alternativa que cal considerar en aquestes
situacions, ja que el funcionament d'aquestes xarxes no depèn de l'existència d'una
infraestructura de telecomunicacions, sinó que la connectivitat s'hi aconsegueix a
través de la cooperació organitzada dels usuaris.

Aquesta tesi de recerca se centra en aquest tipus de xarxes, i té com a objectiu millorar la difusió d'informació en xarxes oportunistes tot analitzant les principals causes que influeixen en el rendiment de la transmissió de dades. Les xarxes oportunistes no depenen d'una topologia fixa, sinó del nombre i la mobilitat dels usuaris, del tipus i la quantitat d'informació generada i enviada, i de les característiques físiques dels dispositius mòbils que els usuaris tenen per a transmetre les dades. La combinació d'aquests elements influeix en la durada del temps de contacte entre usuaris mòbils, i afecta directament la probabilitat de lliurament d'informació.

Aquesta tesi comença amb un estudi exhaustiu de l'estat de la qüestió, en què presentem les contribucions més importants relacionades amb aquesta àrea i les solucions oferides per a l'avaluació de les xarxes oportunistes, com ara models de simulació, protocols d'encaminament o eines de simulació, entre d'altres. Després de mostrar aquest ampli panorama, s'avalua el consum dels recursos dels dispositius mòbils que afecten l'acompliment de les aplicacions de xarxes oportunistes, tant des del punt de vista energètic com de la memòria. 
A continuació, analitzem l'acompliment de xarxes oportunistes considerant tant els entorns de vianants com els vehiculars. Els enfocaments estudiats inclouen l'ús de nodes fixos addicionals i diferents tecnologies de transmissió de dades per a millorar la durada del contacte entre dispositius mòbils.

Finalment, proposem un esquema de difusió per a millorar el rendiment de la transmissió de dades basat en l'extensió de la durada del temps de contacte, i de la probabilitat que els usuaris col-laboren en aquest procés. Aquest enfocament es complementa amb la gestió eficient dels recursos dels dispositius mòbils. 


\section{Contents}

Acknowledgements vii

Abstract $\quad$ ix

List of Figures $\quad$ xix

List of Tables $\quad$ xxii

1 Introduction 1

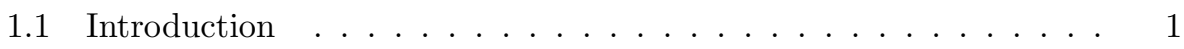

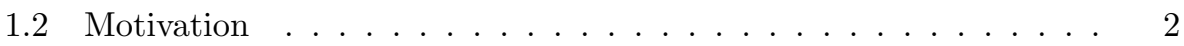

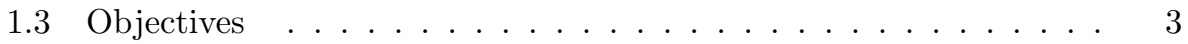

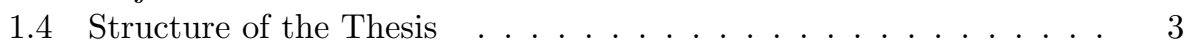

2 State of the Art 5

2.1 Introduction . . . . . . . . . . . . . . . . 5

2.2 Opportunistic Networks: Definition . . . . . . . . . . . . 5

2.3 Performance Evaluation of Opportunistic Networks . . . . . . . . 6

2.4 Simulation Models . . . . . . . . . . . . . . . . . . . 9

2.5 Propagation Protocols . . . . . . . . . . . . . . . 17

2.6 Performance Metrics . . . . . . . . . . . . . . . . . . . . . . . . . . 19

2.7 Simulation Tools . . . . . . . . . . . . . . . . . . . . 20

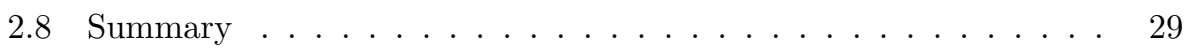

3 Power Consumption 31

3.1 Introduction . . . . . . . . . . . . . . . . . 31

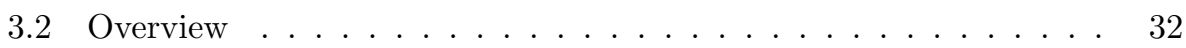

3.3 Power Consumption Measurement Proposal . . . . . . . . . . . . . 32 
3.4 Description of Experiments . . . . . . . . . . . . . . . . 34

3.5 Performance Evaluation . . . . . . . . . . . . . . . . . 37

3.6 Summary ........................ 41

4 Buffer Management $\mathbf{4 3}$

4.1 Introduction . . . . . . . . . . . . . . . . . . 43

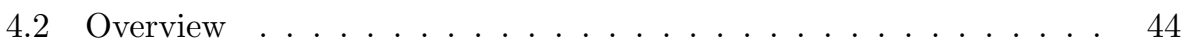

4.3 Buffer Management Proposal . . . . . . . . . . . . . . . 45

4.4 Description of Experiments . . . . . . . . . . . . . . . . . . . . . . . 47

4.5 Performance Evaluation . . . . . . . . . . . . . . . . . 49

4.6 Summary ....................... 56

5 Use of Long Range Technologies $\quad \mathbf{5 7}$

5.1 Introduction . . . . . . . . . . . . . . . . 57

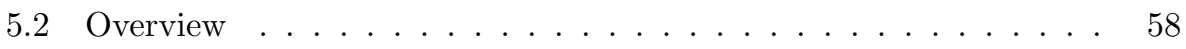

5.3 Long-Range Data Transmission Proposal . . . . . . . . . . . . . 60

5.4 Description of Experiments . . . . . . . . . . . . . . . . 60

5.5 Performance Evaluation . . . . . . . . . . . . . . . 61

5.6 Summary ....................... 66

6 Inclusion of Fixed Nodes $\quad 67$

6.1 Introduction . . . . . . . . . . . . . . . . 67

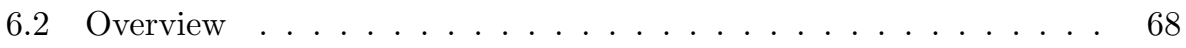

6.3 Message Diffusion Proposal . . . . . . . . . . . . . . 69

6.4 Description of Experiments . . . . . . . . . . . . . . . . . . . . 73

6.5 Performance Evaluation . . . . . . . . . . . . . . . . . . . 74

6.6 Summary ......................... 81

7 Impact of the Data Transfer Time $\quad 83$

7.1 Introduction . . . . . . . . . . . . . . . . 83

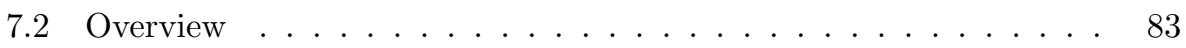

7.3 Message Diffusion Approaches . . . . . . . . . . . . . . 86

7.4 Description of Experiments . . . . . . . . . . . . . . . 87

7.5 Performance Evaluation . . . . . . . . . . . . . . . . . 89

7.6 Summary . . . . . . . . . . . . . . . . . 98

8 Improving the Message Diffusion Performance $\quad 99$

8.1 Introduction . . . . . . . . . . . . . . . . . . . . . . . . . . . . . . . . 109

8.2 Related Efforts . . . . . . . . . . . . . . . . . . 100

8.3 Forced-Stop: Feasibility of a new Message Diffusion Approach . . . 101

8.4 Performance Evaluation of Forced-Stop . . . . . . . . . . . . . . . 103

8.5 The Friendly-Sharing Approach . . . . . . . . . . . . . . . . . . . . 112

8.6 Performance Evaluation of Friendly-Sharing . . . . . . . . . . . . . 114 
Contents

8.7 Summary ........................ 126

9 Conclusions, Publications, and Future Work $\quad \mathbf{1 2 7}$

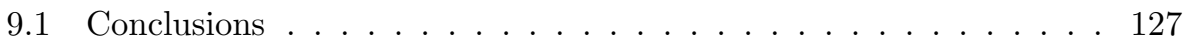

9.2 Publications . . . . . . . . . . . . . . . . . 130

9.3 Internship . . . . . . . . . . . . . . . . . . . . . . . . 135

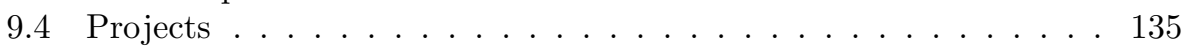

9.5 Future Work ................... 136

$\begin{array}{ll}\text { A Acronyms } & 137\end{array}$

$\begin{array}{lr}\text { Bibliography } & 141\end{array}$ 



\section{List of Figures}

2.1 Performance evaluation process for opportunistic networks. . . . . . . 7

2.2 Accuracy versus cost of the different methods of performance evaluation. Note that the scale of the axis are figurative. . . . . . . . . . 9

2.3 Comparison among transmission technologies for long distances (Figures from $[96,1]) . \ldots \ldots \ldots \ldots$. . . . . . . . . . . . . . . . 15

2.4 Propagation protocols according to the number of relayed messages. . 18

2.5 Overview of The ONE (Figure from [84]) . . . . . . . . . . . . . . 21

2.6 Overview of Adyton. . . . . . . . . . . . . . . . . . 25

3.1 Floating Content scenario. . . . . . . . . . . . . . . . . . . 33

3.2 Devices and tools. . . . . . . . . . . . . . . . . . . 35

3.3 Current consumption over time for different operational states. . . . . 36

3.4 Power consumption by stages. . . . . . . . . . . . . . . . 38

3.5 Impact on the battery performance. . . . . . . . . . . . . . . 40

4.1 Local buffer dropping and forwarding policies. . . . . . . . . . . . . . . 46

4.2 Number of contacts at each simulation hour. . . . . . . . . . . . . . 49

4.3 Delivery probability versus latency for a 12 and 24 hours TTL with different buffer sizes and queue policies using the pedestrian trace. . . 53

4.4 Delivery probability versus latency for a 12 and 24 hours TTL with different buffer sizes and queue policies using the vehicular trace. . . . 54

4.5 Overhead results: buffer occupancy and forwarded bytes . . . . . . . 55

5.1 Vehicular opportunistic network components and diffusion scheme. . . 59

5.2 Rome trace sample of $6 \mathrm{~h}$ of GPS traces. Total length of trace is 30 days, 316 taxis in an area of $10,000 \mathrm{~km}^{2}\left(0.0316\right.$ nodes per $\left.\mathrm{km}^{2}\right)$. . .

5.3 Number of contacts per hour generated by the simulation for each transmission technology. . . . . . . . . . . . . 
5.4 Average delivery success ratio and latency. . . . . . . . . . . . . 64

5.5 Overhead results: Buffer occupancy and forwarded bytes . . . . . . . 65

6.1 Different roles of the cabs and fixed nodes. . . . . . . . . . . . 71

6.2 Criteria to place the fixed nodes. . . . . . . . . . . . . . . 72

6.3 WiFi interfaces embedded in vehicles. . . . . . . . . . . . . . . 74

6.4 San Francisco trace sample of $6 \mathrm{~h}$ of GPS traces. Total length of trace is 24 days, 536 taxis in an area of $1,600 \mathrm{~km}^{2}\left(0.335\right.$ nodes per $\left.\mathrm{km}^{2}\right) \quad$. 76

6.5 Contacts per hour generated by the simulation for each taxi trace. . . 77

6.6 Average delivery success ratio, latency and overhead. Mobile nodes act as sources and destinations, and fixed nodes as relays. . . . . . . . . 78

6.7 Average delivery success ratio, latency and overhead. Mobile nodes act as relays, and fixed nodes are the sources and destinations of messages. 79

6.8 A network with 100 nodes with different percentages of mobile against fixed nodes. There are always exactly 30 nodes producing data in the network and random destinations. The scenario is San Francisco. . . .

7.1 The ONE simulator structure, where the red boxes indicate the modified modules. (The figure is based on the original from [85]). . . . . . 87

7.2 Representation of The ONE simulator running. . . . . . . . . . . . . 90

7.3 Dynamics of the message diffusion, according to movement models. . . 91

7.4 Dynamics of the message diffusion, according to movement models. . . 92

7.5 Message $1 k B$ diffusion according to size, density, and movement models. 93

7.6 Message $1 M B$ diffusion according to size, density, and movement models. 94

7.7 Message 10MB diffusion according to size, density, and movement models. 95

7.8 Percentage of aborted and relayed messages. . . . . . . . . . . . 96

8.1 The ONE simulator structure, where the red boxes indicate the modified modules. (The figure is based on the original from [85]). . . . . . 102

8.2 The ONE simulator running with the NCCU traces. . . . . . . . . . . 104

8.3 Message diffusion. . . . . . . . . . . . . . . . . . . . 105

8.4 Average delivery success ratio by hour. . . . . . . . . . . . . 106

8.5 Delivery success ratio and Latency depending on buffer size (with xaxis in $\log$ scale $)$. . . . . . . . . . . . . . . . . . . 108

8.6 Delivery success ratio and Latency depending on buffer size (with xaxis in $\log$ scale) . . . . . . . . . . . . . . . . . . . 109

8.7 Overhead results: Buffer occupancy and forwarded bytes . . . . . . . . 110

8.8 Several screenshots of the GRChat app. (a) A typical chat conversation; and (b) status of message interchange, showing the remaining time for end the transmission.

8.9 Contact graph for different time intervals of the trace: 3 (a), 6 (b), 12 (c), and $24(\mathrm{~d})$ hours. . . . . . . . . . . . . . . 116

8.10 Average node speed at each hour of the movement trace. . . . . . . 117 
8.11 Average speed and number of contacts for all nodes at each hour of the movement trace. . . . . . . . . . . . . . . . . . 118

8.12 Delivery probability versus latency for a 12 and 24h TTL (Time To Live) with different buffer sizes and queue policies. . . . . . . . . . . . 119

8.13 Average delivery success ratio and latency. . . . . . . . . . . . . . . . 121

8.14 Overhead results: Buffer occupancy and forwarded bytes. (a) maximum of the average buffer occupancy from each node; and (b) average bytes daily forwarded per node ( $y$-axis in log scale). . . . . . . . . . . 124

8.15 Delivery ratio (left) and latency (right) for different contact stop probabilities (16s max. stop). . . . . . . . . . . . . . 125 


\section{List of Tables}

2.1 Summary of performance techniques . . . . . . . . . . . . . . 10

3.1 Logs Structure. . . . . . . . . . . . . . . . . . . 35

3.2 Power Consumption . . . . . . . . . . . . . . . . . . 38

4.1 Simulation parameters to evaluate queue management policies. . . . . 48

4.2 Delivery probability for different TTL, buffer sizes, and queue policies using the pedestrian trace. In bold type are the best results for each

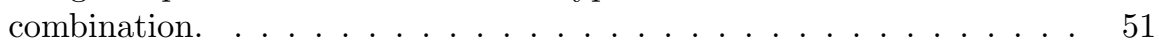

4.3 Delivery probability for different TTL, buffer sizes, and queue policies using the vehicular trace. . . . . . . . . . . . . . . 52

5.1 Simulation parameters varied to evaluate message diffusion. . . . . . . 60

6.1 Simulation parameters varied to evaluate message diffusion. . . . . . . 73

7.1 Simulation fixed parameters. . . . . . . . . . . . . . . . . 88

7.2 Simulation variable parameters. . . . . . . . . . . . . . . . . . . 89

8.1 The main simulation parameters. . . . . . . . . . . . . . . . 104

8.2 Message statistics with a TTL of $12 \mathrm{~h}(\mathrm{x} 1,000) \ldots \ldots \ldots$. . . . . . . . . . . . . . . 11

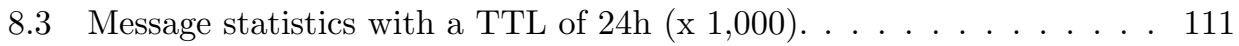

8.4 Simulation parameters varied to evaluate queue management policies. 118

8.5 Delivery probability for a 12 hour TTL with different buffer sizes and queue policies. . . . . . . . . . . . . . . 120

8.6 Delivery probability for a 24 hour TTL with different buffer sizes and queue policies. . . . . . . . . . . . . . . . . . 122

8.7 Simulation parameters varied to evaluate wait time. . . . . . . . . 122 


\section{Chapter 1}

\section{Introduction}

\section{$1.1 \quad$ Introduction}

The use of mobile devices has increased significantly in the last decade, due to the massive use of social networks applications that facilitate the interaction between mobile phone users. By 2017, the mobile phone user penetration is estimated to be around of the $65 \%$ of the world population. Nevertheless, the implementation of the mobile communication infrastructure has not grown at this rate. Moreover, this infrastructure has not expanded sufficiently in the rural areas nor has it been strengthened in urban areas.

Actually, there has been a transition from the typical mobile usage (simple voice calls and plain text messages) to new multimedia services and applications that require higher networks capacities. To cope with these new requirements, researchers have focused their efforts on optimizing the use of the physical resources of mobile devices and the communications infrastructure. One of the most promising technologies are Opportunistic Networks, that combines mobileto-mobile wireless communication and network models that allow the dissemination of information when the mobile devices have the opportunity to establish communications among them.

Communications in mobile opportunistic networks take place upon the establishment of ephemeral contacts between mobile nodes using direct communication via Bluetooth or WiFi-Direct instead of using the Internet infrastructure. Based on this concept, new contact-based messaging applications have recently been developed, such as Firechat (https://www.opengarden.com/firechat.html, OpenGarden, California) or Meshme (http://www.meshme.co, Meshme Inc., Delaware). 
Firechat, for example, a messaging application initially meant for music festivals, became popular in 2014 in Iraq due to the government restrictions on Internet use, and after that during the Hong Kong protests. There are other options, such as, Briar (https://briarproject.org) and Netsukuku (http://netsukuku.freaknet.org), and the so called Floating Content approach [78].

In these types of disruptive wireless networks, where the communication between mobile devices is ephemeral, and links are typically asymmetric and unstable, sending and receiving information depends on the mobility of the mobile devices and the opportunity of contacting other devices, as long as they are willing to collaborate. Concretely, the duration of the contacts between the nodes is a key factor in the dissemination of messages: if the contact time is too low, there will not be enough time for nodes to retrieve all pending messages. Moreover, the management of the internal device resources, basically the buffer occupancy, is critical to provide an efficient service.

This research work is focused on the study of opportunistic networks, from a practical perspective. In this thesis we analyze the main problems that affect the performance of these types of networks to improve their efficiency, and we propose viable solutions to be considered in the short term.

\subsection{Motivation}

Since opportunistic networks do not use Internet or wireless infrastructure for the data transmission, this kind of network has become an alternative to be considered in many scenarios: remote geographical areas where the telecommunications systems are not available, when natural or human-origin disasters destroys the communications infrastructure so only device-to-device communications operates, and finally, in saturated places (overcrowded) where mobile networks are inefficient due to network congestion.

Concerning the opportunistic ad-hoc networks, there are a considerable amount of research works, where the authors have proposed analytical models about the messages diffusion, and their performance was analyzed through simulations. Even in some cases, some prototypes of opportunistic networks have been developed and deployed. However, people have not adopted in large scale the opportunistic networks as a mechanism to send information, probably because they ignore how this communication scheme works, how much hardware and time resources are consumed, or simply they are no willing to collaborate in this type of data transmission.

Each of the above reasons is complicated to be deeply analyzed, but taking into account the large use of the mobile devices, and in order to improve the message diffusion and encourage people to use this wireless networks, we have to focus and examine the consumption and optimization of smart-phones resources that allow taking advantage of the short time contact between mobile users, and 
demonstrate that, with just few seconds of contact time is possible to enhance the message dissemination without using a wireless infrastructure.

\subsection{Objectives}

In this thesis, besides offering extensive information of the performance and application of opportunistic wireless networks, the overall objective is the improvement of the information dissemination in this type of wireless networks, which is based on the evaluation of the main factors that impact on the performance of data transmission.

The following specific objectives have been proposed in order to achieve the general goal:

- To analyze the impact of information transfer time on the dissemination of messages, considering the number and different mobility patterns of the users with mobiles devices, in different area sizes and types of messages.

- To evaluate the consumption of hardware resources, and the impact of this consumption on the diffusion of messages, specifically analyzing the policies of management of buffer queues.

- To assess the epidemic data transmission from the perspective of the communication between vehicles in a real context, using long-range technologies for the transmission of data, and using additional fixed nodes located in strategic places.

- To propose a new scheme to improve the data dissemination, considering the mobility, contact time, and the probability to exchange information between mobile nodes.

\subsection{Structure of the Thesis}

This manuscript is organized in nine chapters as follow:

- Chapter 1: We present the purposes, structure and motivation of this research work.

- Chapter 2: Introduces the State of the Art. We describe concepts and relevant proposals used to evaluated opportunistic networks, that serve as reference to this researching work. Moreover, we offer a brief description and comparison of the current tools employed in the simulation process.

- Chapter 3: We present the evaluation of the power consumption of an application for opportunistic networks, using a scenario of floating content. 
- Chapter 4: It has an assessment of the Buffer Management, where we propose and evaluate a mechanism in order to improve the message dissemination. This analysis was done considering a pedestrian and a vehicular environments, using real traces.

- Chapter 5: We analyze the message diffusion in a real vehicular opportunistic context, using long range (LoRa) and short range (WiFi) communication technology.

- Chapter 6: We present a proposal to improve the message propagation using a opportunistic vehicular network, by the inclusion of additional fixed nodes, a set of real traces is also used.

- Chapter 7: We analyze the data transfer time between mobile nodes comparing certain mobility models and taking into account different density of nodes.

- Chapter 8: We present and evaluate a new scheme to improve the data dissemination for opportunistic networks.

- Chapter 9: Finally, this chapter offers the conclusions, contributions and the future work. 


\section{Chapter 2}

\section{State of the Art}

\subsection{Introduction}

In this chapter we present the State of the Art concerning to opportunist networks, starting with basic definitions. Moreover, we analyze models of mobility, traffic, link technology, routing algorithms, and finally presenting a comparisons of the tools employed to execute the simulations.

\subsection{Opportunistic Networks: Definition}

Opportunistic networks are a mechanism of mobile ad-hoc networks that exploit users mobility to provide data sharing. Individual nodes store, carry and forward messages using direct communication between devices; no fixed infrastructure of communication is required. The final goal is to reach the totality of the participating users.

Opportunistic wireless ad-hoc networks $[121,44]$ are categorized by some authors [112] as a subclass of Delay Tolerant Networks (DTNs) [150]. This model is being promoted by the "Internet Research Task Force", and we can find its specifications in http://www.dtnrg.org. Data transmission in DTN is based on messages or bundles, which are received and then forwarded by nodes. This method is known in networking as Store, Carry and Forward, and it relies on the Bundle Protocol (BP) [161]. Unlike the Internet, in DTN the information delivery time can be extended usually because the communication channels and the data links are intermittent, a phenomenon related to the mobility of transmitters and/or receivers. 
This model is an alternative to be considered in environments where the wireless infrastructure has become inefficient due to the saturation of requests, or when no communication infrastructure is available. Vahdat et al [157] refer to such networks as Partially Connected Networks (due to intermittent contacts), and provide in-depth details about message routing solutions for these wireless networks. For this reason, the design and implementation of protocols and applications for these types of wireless networks has become a challenge for researchers.

There are many different scenarios which exploit the opportunistic networks concepts, like: rural communications, space communications, under-water communication, etc. A good overview of such applications is provided by Mota et al in $[106]$. In $[25,166,139]$ the reader can find complete reviews relative to routing and data dissemination techniques.

Some of the applications are conceptually not different from what we defined above, since communications occur only when devices meet. However, mobility models could be very different, e.g. in the case of space communications and satellite movement.

Routing protocols for opportunistic communication environments enable the storing, carrying, and forwarding of the information between mobile devices. We can find a wide characterization of these protocols in different research works, i.e. $[152,67,36,6]$. The Epidemic protocol [157] belongs to this family of protocols, and its operation is based on the epidemic spreading of messages.

Concerning the assessment of DTN protocols, there are many interesting works, like $[143,34,88,102]$, where the authors analyze the performance of the main routing protocols, namely: Epidemic, PRoPHET [95], Spray and Wait [145], and MaxPro [19]. Beside the algorithm used to deliver the bundles, they consider some key factors like: the message size, the diffusion time, and the overhead, among others.

In the Epidemic model, the contact time between nodes is a key factor in the dissemination of messages. If the contact time is too low, there will not be enough time for nodes to retrieve all pending messages. However, if the contact time between nodes is large enough, the message could be transferred from one node to another with a higher probability of delivery, contributing to the success of message spreading among the majority of nodes in the network.

\subsection{Performance Evaluation of Opportunistic Networks}

Performance evaluation can be defined as quantifying the service delivered by a computer or a communication system [92]. In order to perform an evaluation, we must define our evaluation goals and then the system, the load and the metrics.

First, we must define our goals. It can be obvious, but establishing clearly these evaluation goals, is critical to this evaluation process, and will help in setting the type of evaluation, defining the system and the load, and selecting the metrics to 


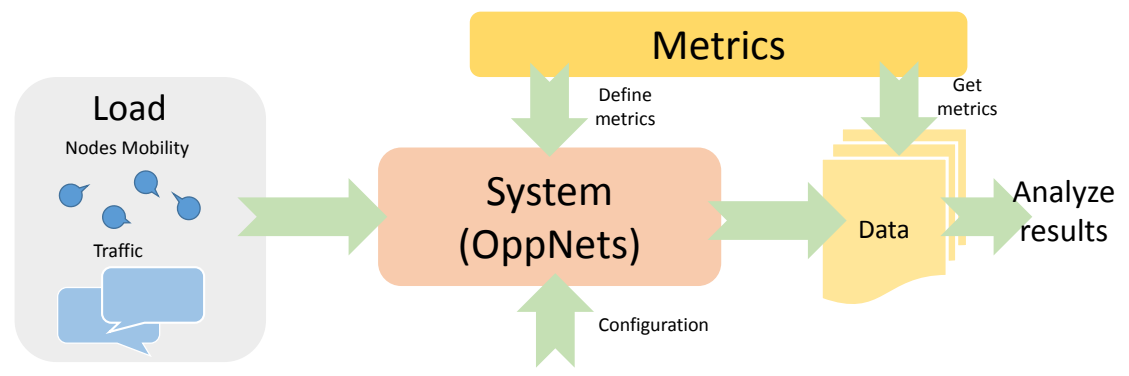

Figure 2.1: Performance evaluation process for opportunistic networks.

obtain. For example, the goal can be the comparison of different data propagation protocols, so we must evaluate them in different scenarios and under different loads in order to determine in which contexts they perform better. On the other hand, if our goal is designing a real new infrastructure, the evaluation must be performed using a load similar to the expected one in the real deployment scenario.

The performance evaluation process consists of three main elements as shown in Figure 2.1. In detail, we have:

- System. The system, in our case, is the opportunistic network to be evaluated and comprises all its elements from hardware to software that can affect the performance of these networks, such as communication range, data transmission speed, buffer capacity, overhead, user behaviour, etc.

- Load. The load (or workload) represents the type and quantity of requests in a system, that in opportunistic networks are primarily mobile nodes with devices that transmit messages. More precisely, we can distinguish between:

- Nodes mobility: defining the movement of the nodes and their interactions are especially important for the evaluation of opportunistic networks. Nodes, movements are usually restricted to the scenario to evaluate. The complexity of these scenarios can range from simple restricted square areas to realistic map-based scenarios. This topic will be further described in Section 2.4.1.

- Traffic load: that is, all messages and network requests generated by the nodes. This traffic load can be as simple as the diffusion of one message to all the nodes in the network, to more complex messaging patterns resembling the use of social or messaging mobile applications. Section 2.4.3 details traffic models further.

- Metrics: Finally, we also need to define the metrics to evaluate the performance of opportunistic networks. These metrics are mainly focused on 
the evaluation of the information diffusion in terms of the diffusion/dissemination time, delivery rate or number of hops. Regarding the nodes and network infrastructure utilization, we can also obtain metrics about buffer occupation, network overhead, energy consumption, etc. When the performance evaluation is done, we usually have a large amount of data that we must process in order to get the desired metrics to proceed on the analysis of the results. Regarding the statistical nature of the metrics we can only obtain deterministic values (for example, a mean), or we can determine its stochastic distribution. This will be discussed in more details in Section 2.6

The characteristics of the previous elements depend on the evaluation technique. We can distinguish three main methods:

- Measuring real or testbed systems: Experiments performed using real scenarios and equipment can be very expensive and sometimes impossible to perform. Nevertheless, some complete evaluations in controlled places have been performed $[12,160,136]$. Other experiments are focused on obtaining traces about nodes mobility. The Crawdad repository [155] is considered the best source to obtain such data.

- Simulation: It is usually a simplified model of the system and the load implemented by software. A common approach is to combine a network simulation tool with realistic mobility traces, in order to reproduce the real dynamics and interaction of mobile nodes. Nevertheless, this simulation can be very computationally intensive (specially for large or complex loads) and its parameterization is not trivial.

- Analytical models. It is a mathematical model of the system and the load. Analytical models can avoid some of the drawbacks of simulation and real testbeds providing a faster and broader performance evaluation. Analytical models usually require strong assumptions or simplifications about the system to evaluate and the load model considered is very simple (usually the diffusion of one message in a network of nodes with a given contact pattern). These models are considered macroscopic models, where we can identify the key mechanisms underlying the information diffusion. Two main classes of analytical models have been proposed for modeling this network dynamics: Markovian models $[52,53,144,56,80,164]$ and deterministic models based on population or epidemic processes [53, 172, 33, 169, 61].

The main problem with Markov chain models is that, if no closed-form expressions are obtained, their numerical resolution when the number of nodes is high is unfeasible, and so they cannot be used to model crowded places. Analytical models require anyway a precise and concise description of the 


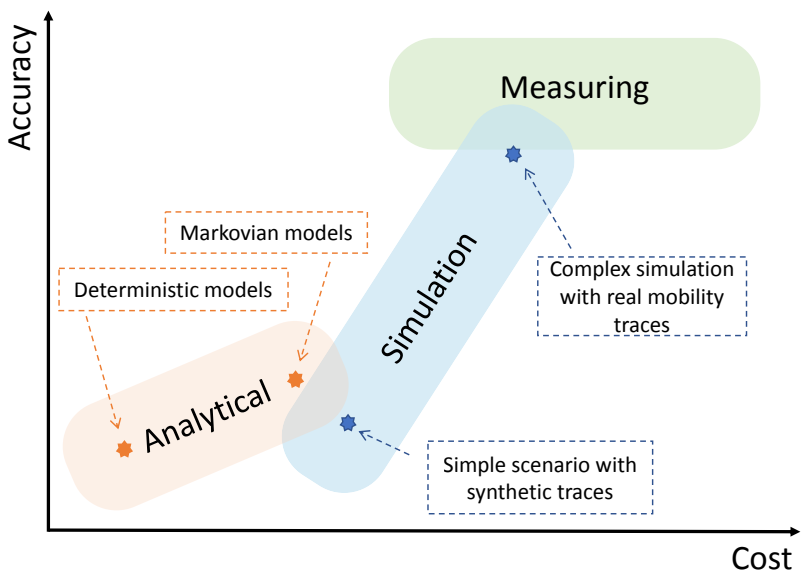

Figure 2.2: Accuracy versus cost of the different methods of performance evaluation. Note that the scale of the axis are figurative.

mobility scenario, that usually assume that the inter-contact times distribution between pairs of nodes is exponentially distributed with a given contact rate $[52]$.

We summarize the main performance methods in Table 2.1. One of the most significant aspects of selecting the type of evaluating method is its precision and cost. In Figure 2.2 we can see the relation between these two factors. On one axis, we have the cost, used as a broader term, comprising both the computation cost (more specifically for simulation and analytic models) and the economical cost (that can be very high in real experiments). On the other axis, we have the accuracy of the obtained results compared to the realistic ones. Analytical methods are very fast, but the results can be unrealistic. However, simulation can obtain a precision very close to real testbeds using complex simulators and real mobility traces, despite its computational cost. Thus, the most of the performance analysis in this thesis was done using different simulation tools.

\subsection{Simulation Models}

The experiments of this thesis were done mainly using simulators. Now, we introduce the most relevant simulation models for opportunistic networks, that are: mobility models, radio transmission models, traffic models, and networking (protocols) models. In this section we explore each of these families in terms of available models and their properties. The upcoming paragraphs give an executive overview 
Table 2.1: Summary of performance techniques

\begin{tabular}{|c|c|c|c|}
\hline & $\begin{array}{l}\text { Measuring (real } \\
\text { system) }\end{array}$ & Simulation & Analytical \\
\hline System & Real & $\begin{array}{l}\text { Depends on the } \\
\text { simulator, from } \\
\text { simple to complex }\end{array}$ & $\begin{array}{l}\text { Simple. Strong } \\
\text { assumptions and } \\
\text { simplifications. }\end{array}$ \\
\hline Load & $\begin{array}{l}\text { Real or synthetic } \\
\text { (system } \\
\text { benchmarking) }\end{array}$ & $\begin{array}{l}\text { Very configurable: } \\
\text { from simple loads } \\
\text { (synthetic mobility } \\
\text { models and simple } \\
\text { scenarios) to } \\
\text { realistic loads } \\
\text { (map-based } \\
\text { scenarios, } \\
\text { trace-based node } \\
\text { mobility) }\end{array}$ & $\begin{array}{l}\text { Very simple (contact } \\
\text { rate, simple area, no } \\
\text { spatial } \\
\text { consideration, one } \\
\text { message) }\end{array}$ \\
\hline Metrics & $\begin{array}{l}\text { Measurement of the } \\
\text { system and are } \\
\text { restricted to the } \\
\text { experiment. }\end{array}$ & $\begin{array}{l}\text { Custom metrics } \\
\text { (depends on the } \\
\text { simulator). }\end{array}$ & $\begin{array}{l}\text { Deterministic values } \\
\text { for population } \\
\text { processes, and } \\
\text { distributions for } \\
\text { Markov Chain } \\
\text { models. }\end{array}$ \\
\hline Pros & $\begin{array}{l}\text { The more realistic } \\
\text { method. }\end{array}$ & $\begin{array}{l}\text { Very flexible: full } \\
\text { control of workload, } \\
\text { scenario model, } \\
\text { metrics, etc. with } \\
\text { different resolution } \\
\text { levels. }\end{array}$ & $\begin{array}{l}\text { Give insights of the } \\
\text { system behaviour. } \\
\text { It allows a trade-off } \\
\text { evaluation. } \\
\text { Deterministic } \\
\text { models do not } \\
\text { depend on load size } \\
\text { and are very fast. }\end{array}$ \\
\hline Cons & $\begin{array}{l}\text { Very limited } \\
\text { scenarios (it is not } \\
\text { easy to evaluate the } \\
\text { effect of varying } \\
\text { parameters). } \\
\text { Expensive, we must } \\
\text { construct the } \\
\text { system. }\end{array}$ & $\begin{array}{l}\text { Computation time } \\
\text { depends on the load } \\
\text { and systems } \\
\text { complexity, and can } \\
\text { be very high. }\end{array}$ & $\begin{array}{l}\text { No spatial } \\
\text { consideration. } \\
\text { Results may be not } \\
\text { precise. Markov } \\
\text { Chain models can } \\
\text { be imprecise with } \\
\text { high number of } \\
\text { nodes. }\end{array}$ \\
\hline
\end{tabular}


of available models, without claiming to be an exhaustive classification. We have selected the most relevant models for simulating opportunistic networks.

\subsubsection{Mobility Models}

As mobility is an opportunity for a communication, understanding of the human movement patterns is considered an essential part in order to evaluate the protocol performance under realistic mobility conditions. The opportunity for a communication can be modelled in a simulator considering contacts between users or considering the effect of change in their positions. This subsection focuses on how a position of a user is modelled in simulators, so called mobility models. With other words, the mobility model answers the simple, but very important question: where is the user? It typically follows the general algorithm:

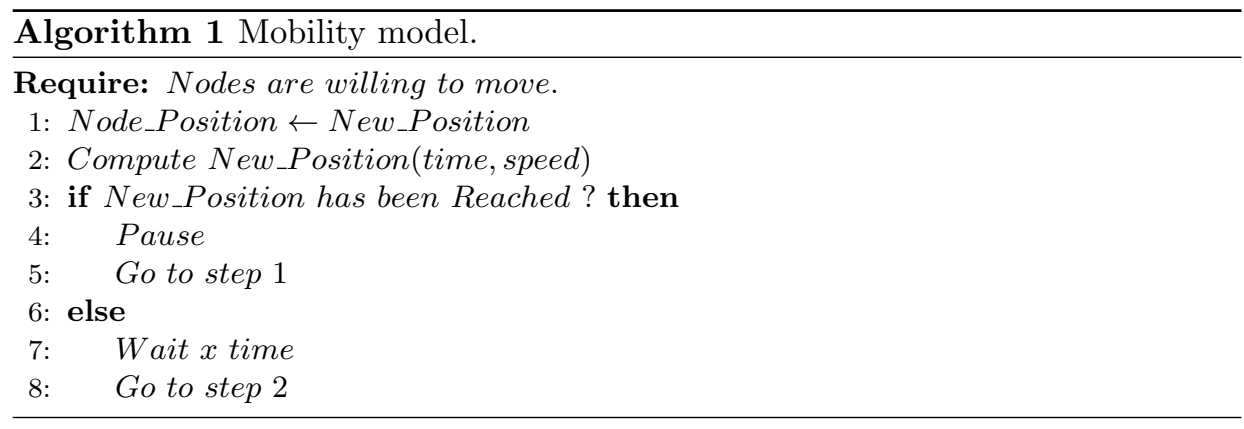

Mobility models can be categorized into three main types. The main difference is step (1) in the above algorithm - how to select the next location (New_Position). However, some also consider different speed of movement.

a) Random Mobility Models. Models with random movements employ stochastic movement patterns to move a node within a given area. The most frequently used mobility model in many simulations is the Random Way Point (RWP) model due to its simplicity of implementation. The mobile node selects a destination randomly and moves towards it with a randomly selected velocity. The other extended mobility models based on RWP can further be characterized as mobility models with temporal dependency (i.e., the next movement will depend on the previous history), with spatial dependency (i.e., tend to change the movement in the correlated manner) and with geographic restriction (i.e., movement is restricted by streets or obstacles). Almost all the random mobility models available are discussed in details in [11] and [22]. However, these models are not useful for opportunistic networks as the mobility patterns used in opportunistic networks should mimic human behaviour where the movement of a user is also influenced by their daily activities (at home, school or work), their means of transportation 
(foot, bike, bus, etc.) or by the behaviour of other users in their neighbourhood/environment. Many of these activities are related to user behaviour and social relationships in addition to the above mentioned three characteristics. We will show these properties and their impact on opportunistic networks performance also experimentally at the end of this section.

b) Real Mobility Traces. There are a large set of datasets obtained from the observation of nodes, mobility in real scenarios. These traces provides accurate information, especially when the trace is being collected among a large number of participants and for a longer period of time. We can find two classes of traces: contact and location based. A contact trace is obtained measuring the times when contacts between pairs of nodes occur for a given time interval. Well known datasets such as Infocom [68], Cambridge [93], Milano [49], MIT (or Reality) [37] among others, have been extensively used, and their statistical properties have been studied in depth $[120,57]$. The simplicity of these traces allows the analytical evaluation and simple simulation of opportunistic networks. The main drawback is that they do not allow to simulate the impact of network layers. Therefore, to evaluate these aspects, we must use location based traces. These traces are the result of obtaining the location of the nodes (mainly GPS coordinates) periodically (or when they move). There are several types of traces, such as the taxis mobility in Shanghai [176], student mobility in a campus [154], among others. Finally, the Crawdad repository [155] contains most of the publicly available traces, and it can be considered the first place to find the required type of traces for simulations. Though trace based mobility models represent very realistic movement patterns, they are not very useful when it comes to the new network environment where the collection of traces has not been performed yet. Furthermore, they are fixed - they cannot be scaled up or extended in time. Also, they cannot react to any changes form user's perspective, e.g. run away from fire. They are also expensive to use in simulations, because the information about next points has to be read from external files. We will show this behaviour at the end of this section.

c) Hybrid Mobility Models. This category shows a combination of the first two described above. In these models, some parameters (e.g., frequency of user movements w.r.t. locations) for a random model are derived based on a collection of traces or based on user experience. For example, Small Worlds In Motion (SWIM) model [104] is based on the assumption that a user either selects as next PoI (Point of Interest) a location close to her home or a very popular location (e.g. a popular restaurant in town). Thus, hybrid models attempt to model real properties of human movements by taking into account "common sense" assumptions, but also statistics from traces. Hybrid models achieve better performance and scalability compared to real mobility traces. 
Many studies are available that aim at providing realistic mobility models based on social relationship (e.g., users, periodic travels over short distances, movements coordinated by social relationships, etc.) between users and location preferences. Some of these models are: TLW (Truncated Levy Walk) [134], SLAW (Self-similar Least Action Walk) [108], SMOOTH [107], SWIM [104], HCMM [17], WDM (Working Day Model) [38], TVC (Time Variant Community model) [64], HHW [170] and SOLAR (Sociological Orbit models) [50].

According to this research work, we can conclude that Random Mobility Models are not suited for simulating opportunistic networks. One should preferably use sophisticated hybrid models, or, if not available, real traces.

\subsubsection{Link Technologies Models}

The link layer as used and referred to in simulations and wireless networks describes a combination of the data link and the physical layer of the OSI model. The objective of the link layer is to adapt the data from the higher layers (i.e., application data) for the used media (i.e., wireless channel). These aspects are important when simulating opportunistic networks since they impact on the delivery rate and delays through factors like buffers management, re-transmissions, connections, etc.

a) Short Range Technologies. The most well known link technology which can be used freely, i.e., without being bound to any operator, is the WiFi technology, which is based on the IEEE 802.11 standard $[71,70]$ defined by the Institute of Electrical and Electronics Engineers (IEEE). Various "specifications" belong to this technology, the most widely known being the 802.11n and the 802.11ac, which are currently available in most of the modern handheld devices. But several other specifications exist which are relevant for opportunistic networks. For example, IEEE $802.11 \mathrm{~s}$ is a standard specifically designed for mesh networks where the participating nodes create a layer two mesh network which can also be described as a kind of MAC-relaying infrastructure. IEEE 802.11ah is a new upcoming standard optimized for energy restricted devices such as sensor nodes and machine-to-machine communication. The focus of IEEE 802.11ah is on supporting a larger number of nodes (up to several thousands) and a lower energy consumption compared to the common WiFi standard. Related to WiFi and relevant to opportunistic networks is WiFi-Direct, a technology that enables WiFi devices to connect directly, allowing an easy pairing of devices for short-term data transmission without the need of an infrastructure. It basically uses the ability of modern handheld devices to become an access point. This technology was developed to overcome the practical limitations of the "Ad-Hoc mode", originally defined in the IEEE 802.11 standard with the same objectives. From the link layer point of view, WiFi-Direct based networks behave as classical WiFi networks. 
Another widely used technology is Bluetooth (standardized as IEEE 802.15.1) in all its variants [15]. Especially with version 4, known as Bluetooth Low Energy (BLE) and with the new features introduced with Bluetooth 5.0, it offers energy efficient functionalities for wireless communication in constrained environments and opportunistic networks.

The main advantage of Bluetooth and $\mathrm{WiFi}$ is their wide availability on a variety of end user devices ranging from smartphone to a plethora of IoT (Internet of Things) oriented devices. However, some other specialized technologies exist which should also be considered for opportunistic networks, since they may become relevant for some specific applications scenarios and environments, such as national park monitoring.

First of all, and referring to the very active area of Wireless Sensor Networks and Smart Things, we would like to highlight IEEE 802.15.4 [72]. Several higher level protocols like ZigBee, WirelessHART and Thread are based on this standard. Its main advantage is the low energy consumption and the optimization for low power devices.

b) Long Range Technologies. Currently, LoRa [97] and Sigfox [140], two standards that fall into the category of $L P W A N$ (Low-power Wide-area network), are becoming more and more widely used in the area of IoT as they claim to have a very long transmission range, up to several kilometres. The low bandwidth offered, the possible scalability issues and the actual benefits of such long transmission ranges are still being observed and tested, but in the future they may become a good option for implementing specific opportunistic networks-based services. We have empathized about LoRa, because in one of the evaluation chapters we use this technology for an message diffusion scheme.

Low-Power, Wide-Area Networks (LPWAN) [131] are a feasible solution to link and support the thousands of devices headed for the Internet of Things (IoT) [73] [98]. Among the LPWAN technologies is LoRa, designed to optimize key aspects such as battery lifetime, capacity, communication range, interference robustness and cost. LoRa is employed in multiple application domains, such as metering, security, and machine-to-machine (M2M) communication. LoRa can reach a range of more than 15 kilometres in a suburban environment and more than $2 \mathrm{~km}$ in a dense urban zone. Its bandwidth goes from 250bps to 50kbps depending on geographical conditions.

LoRa significantly increases the communication range thanks to the chirp spread spectrum modulation. Chirp transmission systems have been used in military activities for several years for long communication distances that can be achieved, and robustness to interference thanks to the modulation which uses the entire channel bandwidth to broadcast a signal.

In order to give an overview of LoRa and for space constrains, we show in Figure 2.3 the main advantages and limitations of LoRa vs. other technologies, in terms 


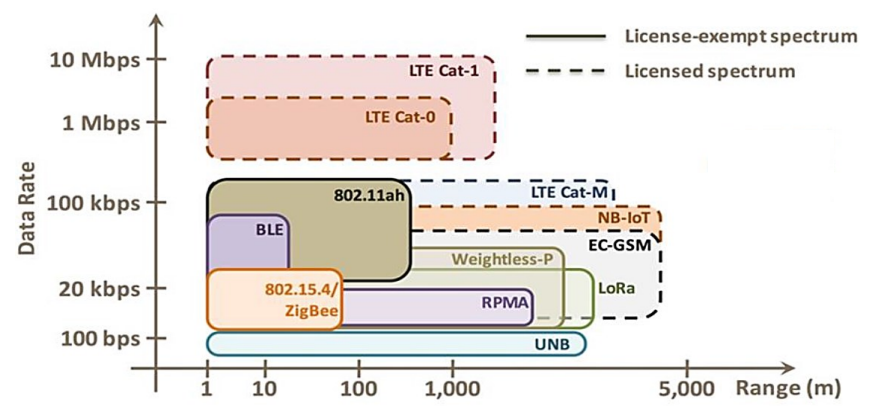

(a) Data rate comparison.

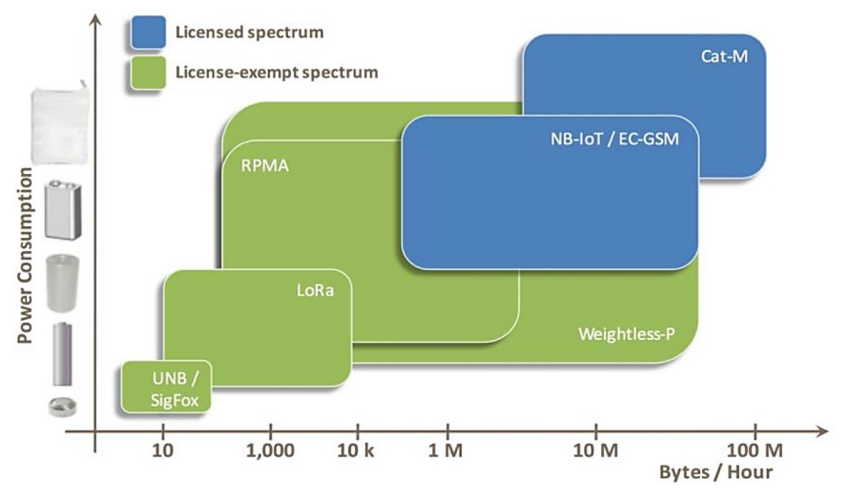

(b) Power consumption comparison.

Figure 2.3: Comparison among transmission technologies for long distances (Figures from $[96,1])$.

of data rate, communications range in the Figure 2.3a, and considering the power consumption and bytes transmitted the Figure $2.3 \mathrm{~b}$. We can appreciate that LoRa is the best alternative in real scenarios where a long distance data transmission is required, with a moderated bandwidth, and low power consumption.

In this work, we are interested in long range communications in vehicular networks. Low power is a bonus but is not a strong requirement in this application because any vehicle could provide more than enough energy.

Some simulators offer realistic implementations of the above described link technologies. WiFi is well supported by OMNeT++ (using INET) and ns-3. Both can simulate Ad-Hoc and mesh networks as well as the classical infrastructure based ones. The new IEEE 802.11ah standard is not officially being supported 
by both simulators. IEEE 802.15 .4 is also supported by OMNeT++ and ns3 where the focus of ns-3 is more on the network layer and IP-based networks (6LoWPAN) whereas OMNeT ++ simulates down to the physical layer. Bluetooth is neither directly supported by OMNeT ++ nor by ns-3. For both simulators, several community driven projects with different qualities exist where the variety of OMNeT++ based ones is higher. Thus, implementing those link technologies is possible and typically requires only good understanding of the standard itself.

The impossibility to completely and exactly model any specific real scenario where various details should be considered, like the presence of physical obstacles, the mobility or the density of the nodes, motivates the use of a so called "ideal link layer" to simulate high-level data propagation opportunistic networks protocols and algorithms. Basically, most of the simulators, including those we explore in this work, assume that if two nodes are in contact, that is if their distance is less than a certain threshold, they can exchange messages. Typically the presence of a contact is re-validated every $x$ seconds. The ONE and Adyton simulators only have ideal link models. ns-3 has only real link technologies and the implementation of an ideal one is not trivial. OMNeT ++ has the potential of offering both environments, but it requires a complete new implementation of the opportunistic networks data propagation protocols.

\subsubsection{Traffic Models}

One of the main parameters when simulating a real-world scenario is how much data is created and circulated in a particular network. Also in this case, the used models should follow the real-world case characteristics as closely as possible. The traffic model is considered very important in opportunistic networks, since the creation time of a particular message is crucial for the resulting delivery delay. For example, if the user creates the message at home with no contacts to other devices, it can only be delivered on the next days, when she starts moving again, thus introducing a high delivery delay.

When talking about traffic models, we need to differentiate between traffic size and traffic frequency. The former models how much data is created at once (e.g. always $1 \mathrm{kB}, 1 \mathrm{~GB}$ or other random sizes), while the latter dictates how often data is created (once per second or week, randomly, etc.). Additionally, it is important to note that there are destination-oriented and destination-less traffic models.

A very good overview of existing models is provided in the book of Wehrle et al [162]. We consider here only some of these models, which are more relevant to opportunistic networks, namely:

- Constant (periodic) traffic: This is the simplest model, where a data packet is created every $x$ time interval. It is considered by many researchers as being not realistic enough but, as we will show later in this section, for 
very large simulations it performs equally well as other models considered more realistic.

- Uniform traffic: This model is also based on a constant $x$ time slot but the data packet is created anytime within the time slot instead of at the beginning (or at the end) of it. The creation instant inside the slot is randomly computed using a uniform distribution.

- Poisson traffic: This is probably the best known model, where the creation instant is randomly computed using a Poisson distribution. It has been shown that this distribution models very closely the traffic in user-driven network traffic, such as web browsing, phone calls, sending text messages, etc.

\subsection{Propagation Protocols}

Routing algorithms for an opportunistic communication environment must be designed to carry out process of information sharing. In [152], the authors perform in first instance a classification according to the utility of each protocol, considering the amount of control information required by each of them. Also, the authors show the chronology of these routing algorithms. In [125] a taxonomy is presented from the perspective of information forwarding. Likewise, [121] proposes a classification according to the use or not of communications infrastructure. As it can be verified, there are several research papers with different criteria to sort the transmission protocols for opportunistic networks. For the interested reader a wider overview of the existing state of the art in the area of opportunistic routing and forwarding, we suggest to refer to the following works [25] or [113].

According to the previous works and the context of analysis of this thesis, we organize the protocols according to the number of copies of forwarded messages, and considering the most important algorithms implemented in the simulator The ONE [85]. The Figure 2.4 shows the above. Below we briefly describe the logic of each of these.

- First Contact [75]. When the nodes are using this routing algorithm, the nodes send messages to the first node they find, until reaching the destination node. A single copy of the message remains on the network. As a disadvantage, the delivery time of the message is high. But the advantage is that a single delivery per message is made.

- Direct Delivery. Like the above algorithm, with Direct Delivery only one copy of each message exists on the network. The strategy of this protocol is to carry the message until it finds its final destination node. It is characterized by being the simplest way to transmit the message to its destination. 


\section{Propagation Protocols}

Single copy

Figure 2.4: Propagation protocols according to the number of relayed messages.

- Epidemic [157]. This algorithm is based on message dissemination, each node broadcasts the message that it receives to the rest of the nodes. But due to the peculiarity of opportunistic networks, each node stores the message for a tolerable time, and then forwards it when it has the opportunity to establish contact with a similar one, and this process is repeated until the package arrives at its final destination. The Epidemic routing protocol, as the name implies, attempts to spread its message to all nodes in the network to ensure that it reaches its target node. The disadvantage of this protocol is the high demand concerning bandwidth and storage memory in nodes, which is technically called buffer. Based on this strategy some variants of this protocol have been proposed $[129,112]$, in which the common denominator is the optimization of the use of resources.

- Spray and Wait [145] is a simple but very effective method based on controlled replication. In this approach the source nodes assign a maximum replication number to a message. A copy of this message is then distributed to a number of relay nodes and the process continues until one of the relay nodes meets the destination or the maximum number of replicas is reached. The idea is very efficient in controlling the overhead of individual messages. Based on this initial strategy many others were proposed that basically focus on improving the efficiency of the data delivery by using different strategies for the replication phase. The best known being the Spray and Wait in binary mode, also some authors refer to as Binary Spray and Wait that, unlike the original version, only sends half of the copies in order to optimize better the resources.

- PRoPHET [95] is the first probabilistic protocol for opportunistic networks. Its strategy is based on the history of contacts, and the same is used to calculate the probability of delivery of a message from a particular node to the destination. This makes the nodes that have frequent encounters have 
a high probability of coming into contact again, predisposing the delivery of the message.

- PRoPHETv2 [95] is a variant of the original protocol, which adds an interconnect time parameter expressed in seconds, which allows to improve the probability of a new meeting by optimizing resources.

- MaxProp [19] spreads messages on the network, but clears them once the copy is delivered to the destination. This protocol sends messages to the other nodes in a specific order keeping in mind the number of jumps and based on the probability, which is calculated according to previous encounters.

- Randomized Rumor Spreading (RRS) [83] is similar to Epidemic and also floods the networks. However, when a node meets other nodes, it randomly selects one item in its cache and sends it out to all neighbours. Thus, it cannot be guaranteed that a useful item is sent out.

- BubbleRap [69] is probably one of the first solutions that focused on using some social aspects of the users' context. They proposed the concept of community and of centrality. When messages are spread, nodes with higher centrality and from the same community are preferred with the assumption that those nodes will meet the destination sooner.

\subsection{Performance Metrics}

Performance metrics provide the means to evaluate the performance of a given system. The type of metrics to use in evaluating a system differs from system to system. Due to the nature of opportunistic networks, commonly used metrics for evaluating networks (e.g., throughput) have a lesser importance than certain others. In the following, a list of widely used metrics are presented together with a brief explanation.

- Delivery Rate: The delivery ratio or rate provides a metric of how many packets were delivered to an intended recipient before the packet was removed from the network. The main reason for the removal of a packet from the network is when the packet reaches its expiration time. There are other reasons as well, such as the removal from caches of the nodes due to the limited size and the caching policy adopted.

- Latency or Delivery Delay: The delivery delay provides a metric of how fast a message can be delivered to an intended recipient (or a group of recipients) considering a per-node or a per-network scope. For example, in case of emergency messages, it is always critical to know the speed of 
data propagation, while in other scenarios, it is important to know how fast the data is propagated to each user depending on different node densities, mobility patterns, user preferences and so on. The timeless of data can be evaluated as Average delay time to receive certain data per node, and Average time to propagate data through the network.

- Overhead: This shows the overhead of the algorithms and protocols used as follows.

- Overhead of irrelevant data and duplicated data per node: In opportunistic networks, nodes receive also uninterested data and duplicated copies. Therefore, the percentage of number of irrelevant data received/sent and number of duplicated copies received with regard to the total number of data received/sent gives an indication of overhead per node.

- Overhead of cache usage: This shows the utilization of the cache for opportunistic networks with regard to the total memory space available in the node. This can be further analyzed with regard to types of data which are propagated. For example, after receiving the data, even though the node is not interested in the data, it might have to store and carry the data until it encounters another node to forward because others might be interested on it. This will cause also an overhead in cache usage for irrelevant data with respect to the preferences of a node.

- Overhead of energy consumption: The percentage of cache usage for irrelevant data will end up by causing overhead of energy consumption as well.

\subsection{Simulation Tools}

In our research work, we will use The ONE and OMNeT++ simulators, but in order to give more information about evaluation methods, we explore the most relevant simulators for opportunistic networks, presenting information about their architecture, usage scenarios and scalability.

\subsubsection{The ONE simulator}

The ONE (Opportunistic Network Environment) [84], is a simulation tool based on discrete events. This simulator was developed in 2009, at Aalto University, and it is now maintained and extended in cooperation between the Aalto University (the Comnet research group) and the Technische Universität Müchen (the Connected Mobility research group). The last version is the 1.6.0, released in July 2016, and is available in the GitHub repository ${ }^{1}$.

\footnotetext{
${ }^{1}$ https://akeranen.github.io/the-one/
} 


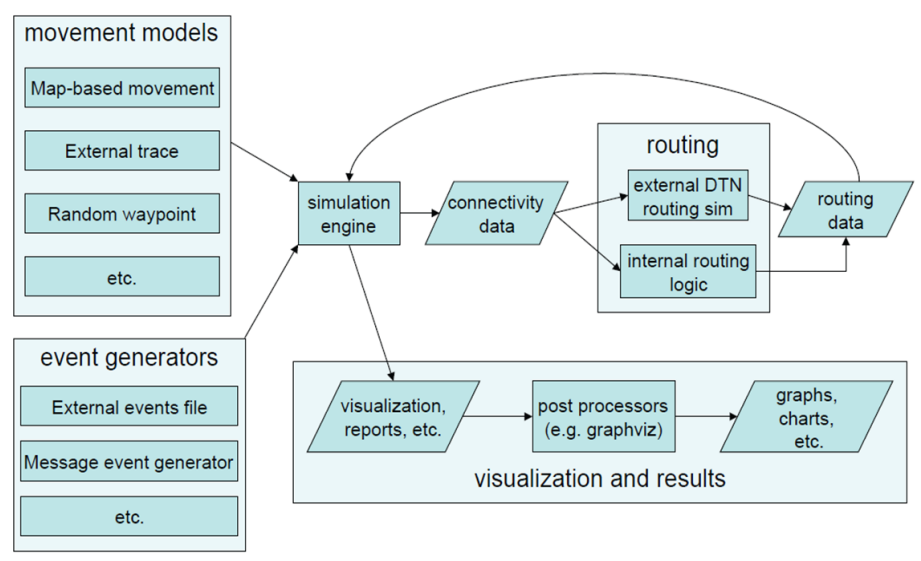

Figure 2.5: Overview of The ONE (Figure from [84]).

This simulator was specifically designed for evaluating the performance of DTN routing and application protocols in wireless networks, see for example[41, 74, $132,133]$. It allows to generate nodes movements using different models, to model messages routing among nodes with various DTN routing algorithms and sender and receiver types and to visualize both mobility and message passing in real time through its graphical user interface. It can import mobility data from real-world traces or other mobility generators. It can also produce a variety of reports such as node movements to message passing and general statistics. This tool is written using the Java programming language, and since the source code is freely available, allows to easily extend it to implement new ideas and solutions and to customize the existing modules.

The flexibility of ONE allows any simulation to manage together several types of wireless nodes, and group these according to their features and possible relationship, i.e., pedestrians, vehicular, or even stationary nodes placed in points of interest. The grouped nodes can share some common parameters, such as the mobility model, the buffers size, the transmission range, etc.

ONE offers an intuitive Graphical User Interface (GUI), where the users can see in a detailed way the scenario settings, and run shorter simulations to validate the set-up; this is actually recommendable before launching a large simulation process. In the case of large and different simulations, where various parameters have to be changed, ONE offers a mechanism called "run indexing" that can be edited in the same configuration file and executed in batch mode. 
ONE offers the possibility to generate synthetic traces based on six main DTN routing protocols namely: Epidemic [157], Spray and Wait [145], Prophet[95], First Contact[75], Direct Delivery [144], and Maxprop [19]. Also, some of their variants are available, such as ProPhet v2 [126], Wave Routing, Maxprop with estimation, and Scheduling routing using Dijkstra's algorithms for shortest path implementation for schedule data.

Respect to mobility models, ONE provides the most relevant models, such as: Random Walk, Random Way Point, Linear, and Grid. In addition, it enables using movements based on maps and routines based on common user patterns, such as Work and Office Day. Moreover, ONE has a module to manage external movements inputs, e.g. synthetic mobility traces generated for other applications, or GPS traces coming from real mobility situations e.g. pedestrians or cars, This data input has to be formatted with a timestamp and their respective coordinates, in order to generate the mobility of the nodes into the simulator.

Finally, ONE also provides the basic procedures to manage the devices, internal buffers.

\section{Limitations}

The ONE simulator requires a lot of resources in scenarios with numerous nodes. To avoid long execution delays, the simulation process is being executed by incremental time steps and all the participant agents play their role through the mobility models, generating contacts and messages, and maintaining data to generate reports. It is therefore recommendable to define a reasonable time step size, without affecting the nodes interactions and avoid losing important data to the final statistical reports.

This simulation tool does not offer support for network lower layers (MAC and physical), these layers are abstracted in the simulator. There are some methods based on interpolations to simulate the possible effects related to the interference and distance-bandwidth.

\subsubsection{OMNeT++}

OMNeT ++ is an object oriented, general purpose discrete event simulator (DES) written in $\mathrm{C}++$. It provides mechanisms to build and simulate wired and wireless networks. The OMNeT ++ simulator itself only provides the building blocks to build nodes in networks that operate using different wired and wireless protocols. There are different $\mathrm{OMNeT}++$ frameworks that have different protocol implementations for users to build and simulate nodes that consist of specific protocol stacks. INET is such a framework that implements the protocols of the Internet Protocol (IP) suite. The INET framework is part of the official OMNeT++ distribution. When considering support for Opportunistic Networks, the INET framework lacks some of the prominent protocols related to simulating nodes in Opportunistic Networks. To compensate for this deficiency, a number of research efforts have concentrated on extending the official frameworks and other 3rd party 
frameworks for OMNeT++ to include protocols and mechanisms. These extensions have stayed unofficial and parts of the code developed have become obsolete due to the changes that have been done in the architecture of $\mathrm{OMNeT}++$ and the official frameworks. In the following paragraphs are some of the most relevant works related to Opportunistic Networks in OMNeT++.

The authors of [54] proposed a set of mechanisms and methodologies to simulate Opportunistic Networks and Delay Tolerant Networks in OMNeT++. Their main focus was on mobility. Their work extended the mobility related components of the Mobility Framework (i.e., a predecessor of the INET Framework) to simulate trace based mobility and node interactions based on contact traces. The code developed by the authors include a toolbox to generate mobility traces based on a given mobility model. The work has used a scenario focused on urban content distribution to evaluate the performance of the proposed mechanisms.

In [55], the work on [54] has been extended to evaluate opportunistic networks with nodes which are equipped with multiple network attachments (e.g., Bluetooth, 802.11, cellular, etc.). These attachments are turned on and off when required to communicate depending on the context. The radios used in the multiple attachments are realized using the MiXiM framework of OMNeT++. The dissemination of data throughout the network is done using a simple data exchange protocol that exchanges data when nodes meet.

The work presented in [89] focuses on caching and relaying strategies to improve distribution of content in opportunistic networks. The dissemination of data is based on a publish/subscribe model where the exchange is performed only for the requested data (i.e., subscribed) with unicast transmissions. The scenarios used for evaluating the strategies are twofold; an outdoor mobile user scenario with high mobility and a subway scenario with entering and leaving users.

The authors of [54], [55] and [89] have made their implementation (called OppoNet) available at GitHub. This code was developed with $\mathrm{OMNeT}++$ version 4.1 (2010).

The authors of [174] have built a model framework in OMNeT ++ to simulate opportunistic networks. This implementation is an extension of the OppoNet work of [54], additionally using the INETMANET framework. The focus of the work is in the forwarding layer of an opportunistic networking node, separating the operations into multiple sub processes. The work introduces the ExOR and MORE protocols comparing the performance against OLSR used in MANET based nodes. The code was based on OMNeT++ version 4.2 (2013).

The authors of [171] have built models in OMNeT ++ to evaluate the performance of physical layer protocols in opportunistic networks. The work extends the MiXiM framework to perform broadcast and changes the fading model to have symmetric signal receptions. The data dissemination protocol used in the work selects a set of nodes to send data based on a priority which is computed using a metric (e.g., closeness to node). The source code of this model (OppSim) is available at SourceForge. The code was based on OMNeT++ version 4.2 (2013). 
Opportunistic Protocol Simulator (OPS) is another OMNeT ++ model for simulating opportunistic networks, developed by some of the authors of this work. It has a modular architecture where different protocols related to opportunistic networks can be developed and evaluated. The current version, which is available at GitHub and implements a simplified version of the Randomized Rumor Spreading (RRS) algorithms [83]. OPS is based on the OMNeT++ 5.0 version (2016) and INET Framework version 3.4.0 (2016).

\section{Limitations}

$\mathrm{OMNeT}++$ does not offer the possibility to transfer simulation models to real implementations, e.g. directly to Linux distributions.

\subsection{3 ns-3}

Network Simulator 3 (ns-3) is a discrete event simulator primarily focused on simulating IP based networks with an emphasis on the network layer (layer 3) and the above layers of the protocol stack. The simulation scenarios can be created using $\mathrm{C}++$ or Python, and are run as command line applications without a GUI. However, NetAnim is a tool shipped with ns-3 for visualizing node mobility during or after a simulation. ns-3 is licensed under the GPLv2 open source license and thus usable by everyone for free. ns-3, which is object-oriented, although it can be considered the successor of ns-2, was completely rewritten. For this reason, ns-3 is incompatible with ns- 2 and the simulation models have to be adapted to be used in ns-3. At present, ns-2 is only lightly maintained as the focus of the developers is on ns-3 which therefore should be used for new projects.

The simulator ns-3 supports simulations of both wired and wireless networks by offering the major protocols for all layers of the Internet protocol stack. All communication modules have been designed in a way that the interfaces can be matched easily to the standard Linux APIs. Thus, it is possible to easily move a simulation setup to the real world and vice versa. It is also possible to interact with existing real-world networks using a virtual TAP (layer 2) devices, connecting simulated and real nodes. Besides the communication-related models, ns-3 offers support for a variety of mobility models such as Random Way Point, Gauss-Markov or trace-based mobility as well as BonnMotion generated mobility patterns. The energy consumption, energy generation and the battery behavior can also be simulated in detail using ns-3.

Besides the core functionalities, ns-3 can be extended easily. For example, the Satellite Network Simulator 3 (SNS3) [128] is an extension to ns-3 to simulate video broadcasting satellite networks. FNCS (Framework for Network CoSimulation) [30] is a co-simulation framework for simulating electrical power grids together with the required communication channels. For communications, FNCS uses ns-3.

As the focus of ns-3 is on the network layer, only a few non-IP based communication implementations exist. For opportunistic networks simulations, we use an 
implementation of the Epidemic routing [8] which is based on WiFi Ad-Hoc and IP traffic. In contrast to the other simulators in this thesis, the Epidemic routing implementation in ns-3 is implemented as an IPv4 routing protocol. The nodes are identified using their IPv4 addresses and thus, the IP protocol stack has to be simulated. This results in increased complexity and thereby increasing simulation times.

\section{Limitations}

A good user interface with debugging and visualization possibilities is clearly missing. Furthermore, the simulator structure is rather complex and not easy to parametrize or change even for experienced researchers.

\subsubsection{Adyton}

Adyton simulator [118], is the newest tool that we are analyzing on this thesis. Similar to The ONE simulator, Adyton is based on events, and it was specially designed to analyze the performance of the routing protocols in wireless opportunistic networks environments. In the simulations process, Adyton can consider another factors such as congestion control, and buffer management mechanisms, which can be combined with more than twenty sets of real on-line traces belonging to different pedestrian and vehicular scenarios. Also, Adyton can work with synthetic traces generated by the BonnMotion tool.

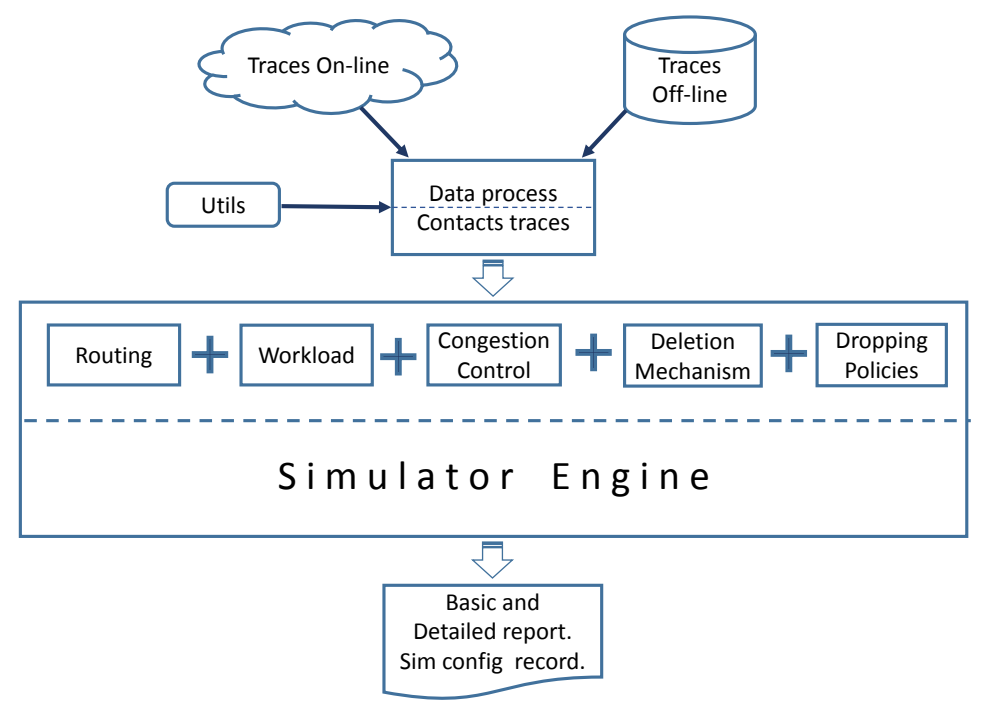

Figure 2.6: Overview of Adyton. 
Between the main protocols that Adyton supports are Epidemic, Direct Delivery, PRoPHET, Spray and Wait, SimBet [32], and BubbleRap [69]. There are more protocols such as LSF, MSF Spray and Wait [147], LSF Spray and Focus [146], Encounter-Based Routing [111], Delegation Forwarding [39], Coordinated Delegation Forwarding [119], and Optimal routing (some of them based on above algorithms) that can be used. Moreover, Adyton has implemented some Congestion Control Mechanisms such as Avoid Overflow, Autonomous Congestion Control (ACC) [20], FairRoute (FR) [127], Buffer Space Advertisements (BSA) [91], Context-Aware Congestion Control (CACC) [163], Congestion Control with Adjustable Fairness (CCAF) [5].

Adyton was developed in $\mathrm{C}++$, and it runs only in Linux operating systems, and the source code is available on the Internet (GitHub). On this repository is explained the Adyton utilization, additional parameterization when specific protocols are used, procedures to implement and integrate more protocols or new functionalities. Furthermore, there are some tools based on scripts that can support previous steps for the simulation process, i.e. generation of synthetic traces and conversion of real traces to Adyton format. Adyton can be executed in interactive mode or batch mode. In the first case the simulation results are shown when the simulation process has ended.

\section{Limitations}

Between the main limitations, we found that this simulation tool does not offer support for network lower layers (MAC and physical), which are abstracted in the simulator. Adyton can not create his synthetic traces, it has to use an external tool to get this type of traces for the simulation process. For the Windows and Mac users, Adyton at the moment can not be executed on these operating systems.

In the future improvements of Adyton should be considered the generation of more reports to get a wide analysis of the opportunistic networks behavior.

Summing up, the utilization of these tools can fill the gap between purely analytical/stochastic and real world trace-driven mobility models. In the next table we contrast the mentioned simulators. 
2.7. Simulation Tools

\begin{tabular}{|c|c|c|c|c|}
\hline Simulator & ONE & ADYTON & OMNET++ & ns-3 \\
\hline Last Version & v1.6.0, 07-2016 & v.1.0, 12-2015 & V5.0, 04-2016 & V3.26 10-2016 \\
\hline OS & $\begin{array}{l}\text { Linux, Windows, } \\
\text { MAC }\end{array}$ & Linux & $\begin{array}{l}\text { Linux, Windows, } \\
\text { Mac }\end{array}$ & Linux \\
\hline Simulator Type & $\begin{array}{l}\text { Discret Events for } \\
\text { DTN and oppor- } \\
\text { tunistic networks }\end{array}$ & $\begin{array}{l}\text { Discret Events for } \\
\text { DTN and oppor- } \\
\text { tunistic networks }\end{array}$ & $\begin{array}{l}\text { Discrete Events, } \\
\text { General purpose } \\
\text { networking }\end{array}$ & $\begin{array}{l}\text { Discrete Events, } \\
\text { General purpose } \\
\text { networking }\end{array}$ \\
\hline License & FreeOpen Source & FreeOpen Source & $\begin{array}{l}\text { Free for Educa- } \\
\text { tion, Comercial } \\
\text { License available } \\
(\text { OMNeST) }\end{array}$ & GPLv2 \\
\hline Source Code & Java & $\mathrm{C}++$ & $\mathrm{C}++, \mathrm{NED}$ & C++, Python \\
\hline GUI & $\begin{array}{l}\text { Yes. Interactive } \\
\text { real-time control } \\
\text { and visualization. }\end{array}$ & No & $\begin{array}{l}\text { Yes. Interac- } \\
\text { tive real-time } \\
\text { visualization. }\end{array}$ & $\begin{array}{l}\text { No. The traces } \\
\text { can be visualized } \\
\text { after the simula- } \\
\text { tion }\end{array}$ \\
\hline $\begin{array}{l}\text { Console / Batch } \\
\text { mode }\end{array}$ & Yes & Yes & Yes & Yes \\
\hline $\begin{array}{l}\text { Continuous devel- } \\
\text { opment }\end{array}$ & Yes & Yes (Newest) & Yes & Yes \\
\hline $\begin{array}{l}\text { Documentation } \\
\text { and examples }\end{array}$ & $\begin{array}{l}\text { Source code is } \\
\text { well documented, } \\
\text { examples, users } \\
\text { communities }\end{array}$ & $\begin{array}{l}\text { Source code is well } \\
\text { documented, ex- } \\
\text { amples. }\end{array}$ & $\begin{array}{l}\text { Examples, } \\
\text { documented. } \\
\text { torials available. }\end{array}$ & $\begin{array}{l}\text { Tutorial available, } \\
\text { source code and } \\
\text { examples are } \\
\text { well documented. } \\
\text { Parts need higher } \\
\text { understanding in } \\
\text { Linux commands. }\end{array}$ \\
\hline Installation & Easy & Easy & Easy & Easy \\
\hline Setup and Use & $\begin{array}{l}\text { Setup: Moder- } \\
\text { ate, Configuration } \\
\text { is realized by text } \\
\text { files, the most of } \\
\text { times centralized } \\
\text { in one file. Use: } \\
\text { Easy }\end{array}$ & $\begin{array}{ll}\text { Setup: } & \text { Moder- } \\
\text { ate. Use: Easy }\end{array}$ & $\begin{array}{l}\text { Easy, Usage } \\
\text { quires } \mathrm{C}++\end{array}$ & $\begin{array}{l}\text { Usage requires } \\
\text { C++ / Python } \\
\text { knowledge. No } \\
\text { UI available, } \\
\text { scenarios have } \\
\text { to be scripted / } \\
\text { programmed. }\end{array}$ \\
\hline $\begin{array}{ll}\text { Network } & \text { Layers } \\
\text { Support } & \end{array}$ & $\begin{array}{l}\text { Application } \\
\text { Layer. The } \\
\text { lower layers are } \\
\text { abstracted. }\end{array}$ & $\begin{array}{l}\text { Application } \\
\text { Layer. The } \\
\text { lower layers are } \\
\text { abstracted. }\end{array}$ & $\begin{array}{l}\text { Lower layers can } \\
\text { be abstracted or } \\
\text { can be connected } \\
\text { to a IP based layer } \\
\text { (with INET) }\end{array}$ & $\begin{array}{l}\text { Lower layers can } \\
\text { be abstracted or } \\
\text { can be connected } \\
\text { to a IP based layer }\end{array}$ \\
\hline Mobility Models & 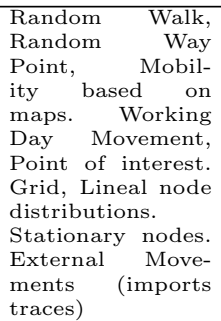 & $\begin{array}{l}\text { Adyton sup- } \\
\text { ports synthetic } \\
\text { traces generated } \\
\text { by Bonn Motion, } \\
\text { works with on-line } \\
\text { real traces belong } \\
\text { to pedestrian, and } \\
\text { vehicles. }\end{array}$ & $\begin{array}{lr}\text { SWIM, supports } \\
\text { synthetic traces } \\
\text { generated by } \\
\text { Bonn Motion }\end{array}$ & 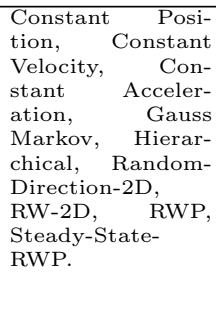 \\
\hline Routing Protocols & $\begin{array}{lr}\text { Supports } & \text { inter- } \\
\text { nal and } & \text { external } \\
\text { routing. } & \text { Exter- } \\
\text { nal: Can read } \\
\text { DTNsim2. Inter- } \\
\text { nal: Epidemic, } \\
\text { Spray And Wait, } \\
\text { Prophet, First } \\
\text { Contact, Direct } \\
\begin{array}{l}\text { Delivery, Max- } \\
\text { prop, and their } \\
\text { variants. }\end{array}\end{array}$ & 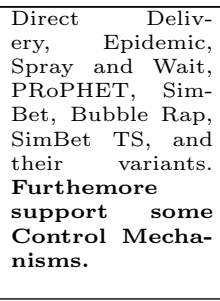 & $\begin{array}{l}\text { Epidemic Rout- } \\
\text { ing, Pub-Sub, } \\
\text { ExOR, MORE, } \\
\text { ODD, RRS }\end{array}$ & $\begin{array}{l}\text { AODV, } \\
\text { DSR, Nix-Vector, } \\
\text { OLSR, Epidemic } \\
\text { (WiP) }\end{array}$ \\
\hline
\end{tabular}




\section{State of the Art}

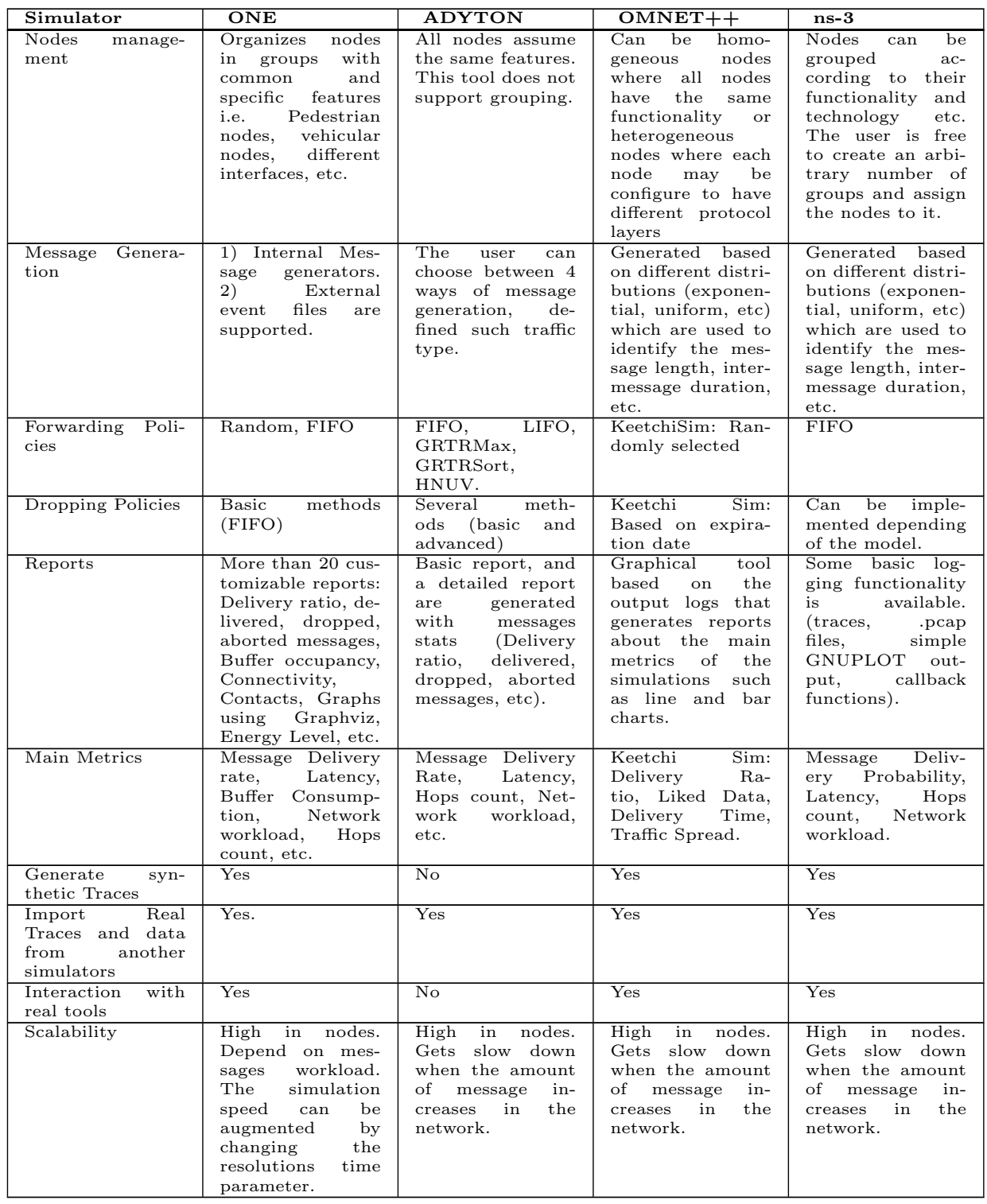




\subsection{Summary}

In this chapter the State of the Art related with the opportunistic networks was presented. Additionally, we have acquired and clarified the methods, procedures, and tools to evaluate the opportunistic networks. Concerning to the simulation tool to be used, we have chosen The ONE simulator for most of our experiments, mainly because this tool is designed specifically to evaluate opportunistic networks, offering several and useful reports, that helped us to analyze the performance of this kind of networks. Additionally, we used OMNeT ++ for a specific evaluation in the Chapter 6. 



\section{Chapter 3}

\section{Power Consumption}

\subsection{Introduction}

The duration of batteries is a key factor that must be considered in the design and deployment of applications and services on mobile devices. The users of this technology are not aware of the energetic impact of the applications executing in their devices, especially if these applications present an adaptive behavior.

The goal of this chapter is to study the power consumption associated to an opportunistic communication application. This will be achieved by analyzing the different operation stages of an application running on mobile devices, using basic statistical analysis calculations on data collected by a logging application. Furthermore, this will allow analyzing the impact of this kind of applications regarding battery performance, providing useful information to users and developers.

In order to make a realistic analysis, we proceeded to study the different operating stages of an opportunistic network application on a mobile device. This allowed us to obtain an approximation of the energy consumption based on the difference of the electrical values of these periods. The experiments were executed in an opportunistic network environment, using a floating content approach as a mechanism for information distribution in a geographically delimited zone.

This chapter is organized as follows: a brief characterization of opportunistic networking is provided in Section 3.2. A description of the experiments and the evaluation procedure are presented in Sections 3.4 and 3.5, respectively. Finally, in Section 3.6 is presented the chapter summary. 


\subsection{Overview}

One of the main problems of mobile devices is the power consumption in the data transmission process, from device mobile discovery to data delivery. In [90] the authors present a quantitative research to save energy through delayed transmission using WiFi and 3G technology. They suggest the activation and de-activation of network interfaces in mobile devices according to specific places. They demonstrate that by delaying the transmission 100 seconds, the achievable throughput and energy gain is less than $3 \%$, whereas with 1 hour or longer deadlines, traffic and energy saving gains increase by $29 \%$ and $20 \%$, respectively. Furthermore, they propose a simulator based on a distribution model and a theoretical framework that enables analytical studies of the average performance of offloading.

The authors of $[3,149]$ present practical and in-depth studies to identify and understand the common factors related to power consumption. They describe the impact of the hardware components utilization on different types of applications, highlighting what, where and how is battery energy being spent. This must be considered when developing applications, especially when applications are running and searching for a particular service offered by another application.

The authors of [10] suggest that applications must be designed with a modular structure to optimize energy resources, and so the required modules would be loaded on demand. Instead, the authors of [100] propose changes at routing protocol levels according to the priority of forwarding nodes, but they discard messages that were sent on minor power nodes. This approach involves extra mechanisms to control reception and transmission. There are other proposals such as [77] that are based on simulations and real traces. In a similar way, they propose communication protocol changes, arguing their effectiveness and low energy properties.

The studies mentioned above provided an overview of recent research related to power consumption on opportunistic networking, and the trend towards the development of models and applications able to exploit the particularities of these wireless networks.

\subsection{Power Consumption Measurement Proposal}

An interesting model for opportunistic communications is Floating Content [79]. In this model, sharing and distribution of information is performed in a limited geographically area called anchor zone. An anchor zone is a geographical location where mobile devices (carried by pedestrians and vehicles) can swap contents in a floating approach, as shown in Figure 3.1. Floating content performance is based on three parameters of the anchor zone $(P, r, a)[114]$ where $P$ is defined as the central point of the anchor zone (this zone is where message exchanging should reach the maximum level). The values of $P$ could be set using GPS or another localization system that is currently available in most smart-phones. 

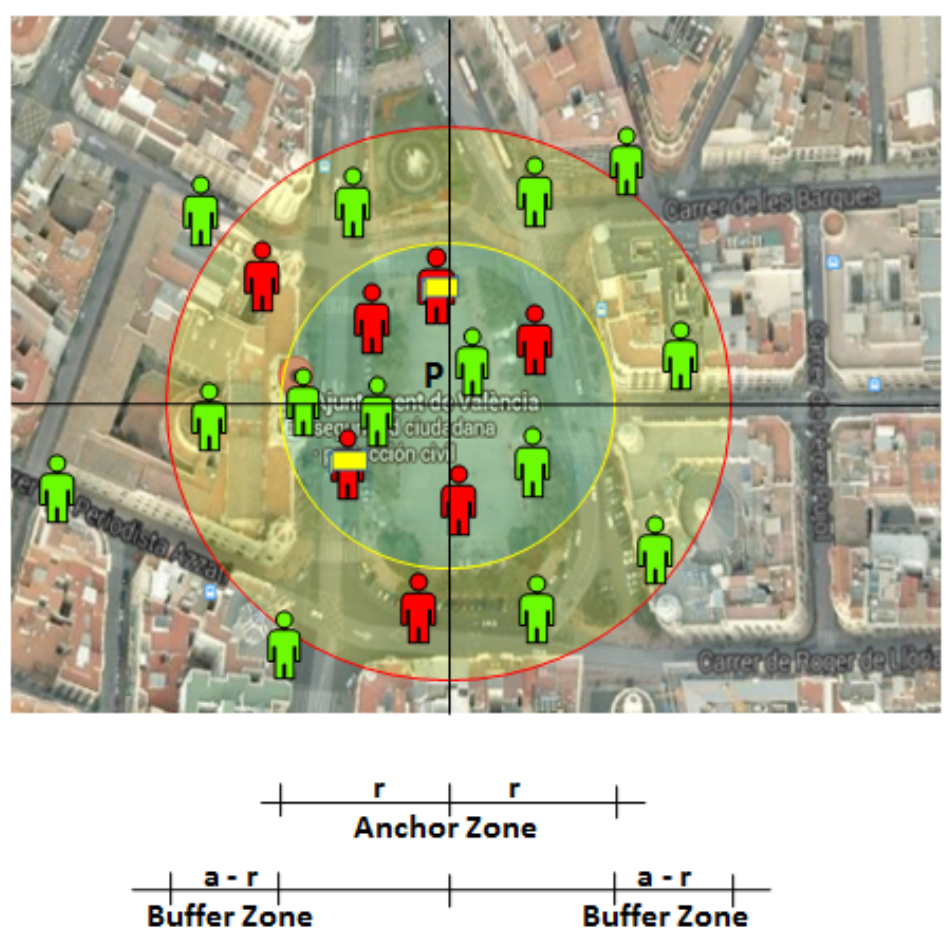

Figure 3.1: Floating Content scenario.

The central circle with radius $(r)$ represents the anchor zone, where some mobile devices can post content, while other can accept and store data copies according their interests, and replicate information if is necessary. This occurs while mobile users are inside this zone. The floating content is also parameterized with a certain lifetime to limit its availability within user devices.

When users are leaving the anchor zone and entering into the buffer zone defined by radius ( $a$ ), the message replication probability decreases since, as mobile devices move away from $P$, the chances that data is erased increases.

Figure 3.1 also shows a realistic location of a city, where red figures represent mobile users with information items (yellow box) to be shared within an anchor zone. Red figures could be users interested in getting and storing data items and likely replicate copies, and green figures are normal users not interested in information.

In order to make a realistic analysis, we proceeded to study the different operating stages of an opportunistic network application on a mobile device. This allowed us to obtain an approximation of energy consumption based on the differ- 
ence of the electrical values of these periods.

The data for the analysis were obtained from a scenario of opportunistic networks using Liberouter under the Floating Content paradigm and as a client device we used an Android smartphone. Liberouter [81] is a result of the research project SCAMPI [138] founded by the European Union. The SCAMPI platform is based on activities of the Delay-Tolerant Networking Research Group (DTNRG) of the Internet Research Task Force (IRTF).

\subsection{Description of Experiments}

This section describes the experimental infrastructure and the procedure used to perform power consumption measurements. First, we present the hardware and networking scheme based on WiFi technology. Second, the measurement methodology is explained. It is organized in phases depending on the sequential activation of opportunistic application functionalities, since every stage has a different power consumption.

The data were captured using a Java-logging application, which was developed specifically for this purpose. Data processing was carried out of the mobile device to avoid increasing the extra power consumption.

\subsubsection{Testbed}

Figure 3.2 shows the hardware and software elements used in the experiment to collect data, organized in two groups:

1. Liberouter [2] is a framework designed to provide opportunistic communications networking. Its operation is based on the Floating Content approach [115]. This networking system consists of a SCAMPI routing platform running a Linux on Raspberry Pi [65] hardware with an USB WiFi dongle $(802.11 \mathrm{~b} / \mathrm{g} / \mathrm{n}$ standard). The deployment of Liberouter is easy and fast; the users just have to install the image in the Raspberry Pi SD card. After turning it On, an open wireless network called LIBEROUTER appears, allowing mobile devices to connect to this access point. Raspberry Pi is a cheap device that allows connecting a wide variety of hardware in its different ports. These features are the main reason to use this type of communication system in our testbed environment.

2. A mobile device composed of a Samsung Smartphone Galaxy SIII NEO running the Android 4.3 operating system. The installed applications are the following: SCAMPI router application (Liberouter), Guerrilla-Pics photo sharing, and Guerrilla Tags messaging app. Furthermore, there is a logging application developed just to collect power consumption data every 0.1 seconds and transmit that information. Table 3.1 shows the structure of 

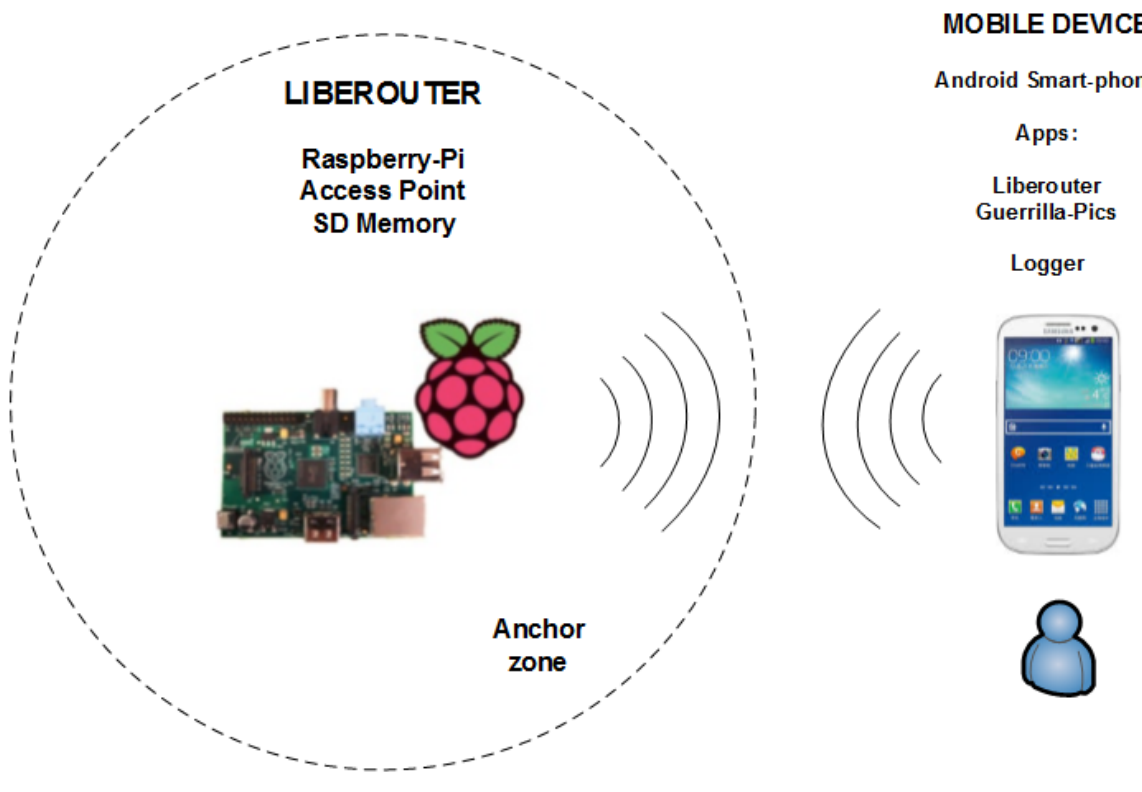

Figure 3.2: Devices and tools.

these records. The mathematical calculations and conversions were done externally.

\subsubsection{Measurement Methodology}

In order to measure the power consumption of Liberouter client apps, the smartphone just powered the WiFi interface, while the GPS and other cellular interfaces were powered off. The logging application acquires data directly from virtual file "/sys/class/power_supply/battery". In this folder we can find text files with updated values of voltage, current and battery state, among others.

Table 3.1: Logs Structure.

\begin{tabular}{|c|c|c|c|c|c|}
\hline No. & Time Stamp & Voltage (V) & Current (mA) & Bytes Tx & Bytes Rx \\
\hline \hline 1 & $19: 14: 29$ & 4.25 & 258.74 & 38 & 48 \\
\hline 2 & $19: 14: 30$ & 4.15 & 298.74 & 150 & 345 \\
\hline 3 & $19: 14: 30$ & 4.26 & 198.44 & 173 & 11 \\
\hline$\ldots$ & $\ldots$ & $\ldots$ & $\ldots$ & $\ldots$ & $\ldots$ \\
\hline
\end{tabular}



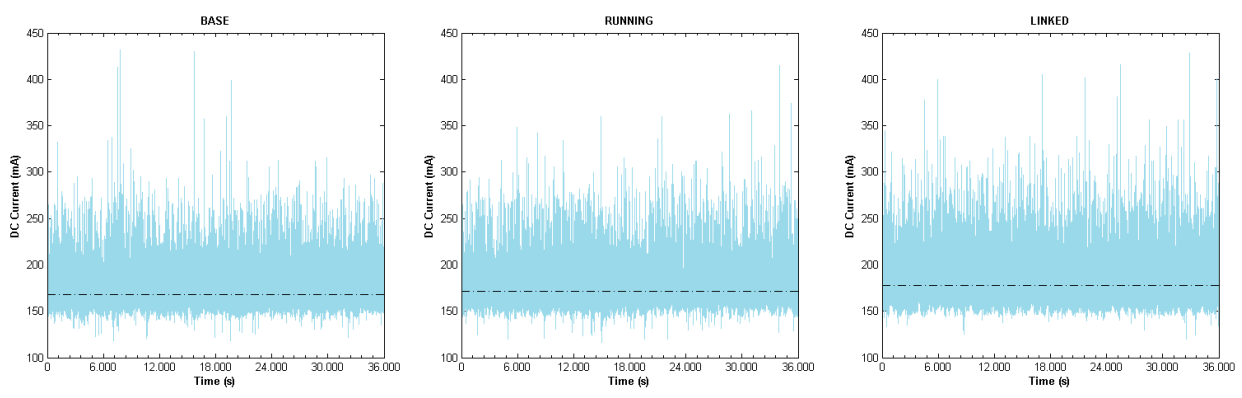

Figure 3.3: Current consumption over time for different operational states.

We addressed the measurements in three sequential operation stages in the mobile device: Base, Running and Linked. The data capture lasted 1 hour in each phase, with a sampling frequency of 0.1 seconds, thereby generating 36,000 records every time. Below we describe the different phases analyzed:

1. In the Base stage, we consider the regular functionality of the smartphone, without any app providing opportunistic networking. Thus, the mobile device has only the basic programs installed.

2. The Running stage takes place when the Liberouter app is running in background without connection to any opportunistic wireless network.

3. The Linked phase takes place when the mobile device is within an anchor zone connected to the Liberouter Access Point, and their protocols are interchanging information every 10 seconds, related to the listening process.

Figure 3.3 shows a graphical comparison of the stages: Base, Running and Linked. We can see the density of the energy consumption according to the mobile device operation. Numerical values are shown in Table 3.2.

Table 3.2 shows the average DC current level on every stage. We used these values to get the DC current of the Liberouter app computing the difference between the mean values for the Running and Base phases.

$$
\begin{aligned}
C_{\text {Liberouter }}= & C_{\text {SRunning }}-C_{\text {SBase }} \\
& 171.18 m A-168.02 m A
\end{aligned}
$$

\section{$3.16 m A$}




$$
\begin{aligned}
C_{\text {Linked }}= & C_{\text {SLinked }}-C_{\text {SRunning }} \\
& 177.34 m A-171.18 m A \\
& 6.16 \mathrm{~mA}
\end{aligned}
$$

According to these results, we can summarize this first section with the following premises:

- The current consumption of Liberouter is $3.16 \mathrm{~mA}$, without establishing any type of contact, as shown in Eq. 1.

- When a mobile device is running the Liberouter app, and it enters into anchor zone, the consumption increases by $6.16 \mathrm{~mA}$ (see Eq. 2) due to information exchanging of communication protocols (in this case TCP). Then, the total consumption is $9.32 \mathrm{~mA}$ when Liberouter is running into the anchor zone.

\subsection{Performance Evaluation}

Now, we conduct an evaluation based on the previous results, in order to calculate the power consumption between the different stages of opportunistic networking, and the influence on the mobile device battery.

\subsubsection{Power consumption during transmission}

In order to calculate the power, we multiplied each record of the collected data by the nominal battery voltage of 3.8 volts, and obtained the average value. We can see in Figure 3.4 the influence of the consumed current by the application. Numerical values are shown in Table 3.2 .

According to these results, the power difference is proportionally the same as for the DC current on every stage: the power consumption of Liberouter app client is $P_{\text {Running }}=12 \mathrm{~mW}$, and when the mobile device is in the anchor zone it grows to $P_{\text {Linked }}=23.41 \mathrm{~mW}$. We can note that the increase is about $200 \%$, since the listening process requires more electrical resources. It is important to remember this factor during the design of services for mobile phones.

While a mobile device is in the anchor zone (Linked), it receives an average of 396 Bytes and sends 290 Bytes every 1 second (this data just belongs to protocol control information). The volume of this information depends on the frequency of emitted beacons.

Once we estimated the power of the Liberouter application in a mobile device, we addressed the same evaluations to know the power consumption when data is 
Table 3.2: Power Consumption

\begin{tabular}{|c|c|c|c|}
\hline No. & Stages & Current (mA) & Power (mW) \\
\hline \hline 1 & $S_{\text {Base }}$ & 168.02 & 638.48 \\
\hline 2 & $S_{\text {Running }}$ & 171.18 & 650.47 \\
\hline 3 & $S_{\text {Linked }}$ & 177.34 & 673.88 \\
\hline
\end{tabular}

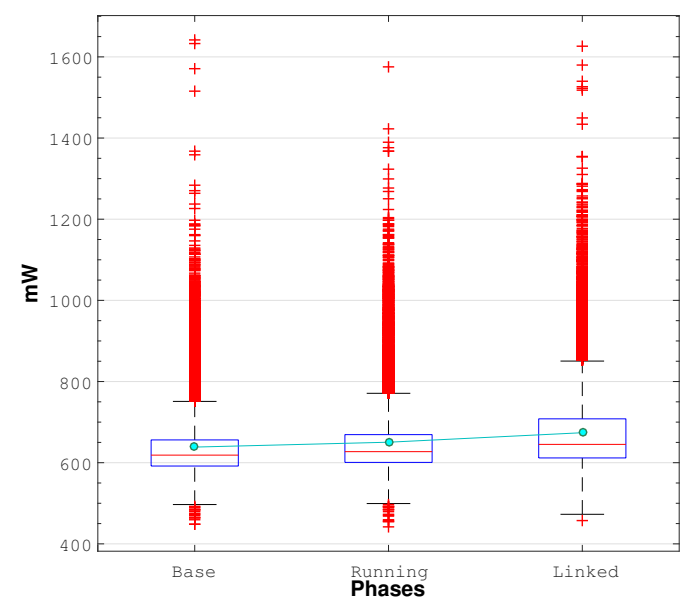

Figure 3.4: Power consumption by stages.

transmitted; then we executed the Guerrilla-Pics (Liberouter app), to send one image file of $96 \mathrm{kB}$, and we got the following values:

- The current consumption is $C_{\text {file }}=40.25 \mathrm{~mA}$

- The power consumption is $152.95 \mathrm{~mW}$.

- The average time to send $96 \mathrm{kB}$ is $58 \mathrm{~ms}$.

According to these values, the energy consumption to send $96 k B$ is 9 mJules.

\subsubsection{Impact on the battery performance}

Finally, we addressed an approximation of the impact of power consumption over time. Then, taking into account the consumption for every phase and the number of transmitted files, the impact on the battery time is calculated using the following expression: 


$$
t_{i}=\frac{Q}{C_{\text {App }}+C_{\text {Linked }} \cdot t_{a}+k \cdot C_{\text {file }} \cdot t_{f}}
$$

where $Q$ is the battery capacity (in this specific case $2,100 m A h$ ); $C_{A p p}=C_{S B a s e}+$ $C_{\text {Liberouter }}, C_{\text {Linked }}$ is the power consumption, $t_{a}$ is the time spent by the mobile device within an anchor zone, $k$ is the number of sent files, and $t_{f}$ is the time required to send a file.

We analyzed two scenarios to explain the impact of the power consumption in the different phases.

In the first scenario, the time is calculated with a fixed value $t_{a}=1$ hour and varying $k$ from 5,000 to 50,000. These transmission rates can reflect, for example, a periodic information update or video transmission. Figure 3.5a shows the results for this scenario, being the blue solid line the relationship between the $C_{A p p}$ consumption and battery capacity. This is the reference value to compare the impact of our target application over time.

The blue dashed line represents the behavior when the mobile device enters in an anchor zone without any data transmission, and the red lines represent the case when mobile users are transmitting. As we can, see the impact of the transmission is lower when the mobile device is linked to an anchor zone. The battery duration is no less than 10 hours.

In the second scenario, the time is calculated by changing the spent time $t_{a}$ by a mobile device within the anchor zone from 1 hour to 8 hours, and $k$ is fixed to 5,000 files. Figure 3.5b shows the result of this approximation. The blue solid lines have the same meaning of the previous figure, but the red lines represent the time spent by a mobile device in the anchor zone. The effect of the file transmission is not significant with respect to the contact and permanence of a mobile user in the anchor zone. In this case, the battery time decreases to near to 9 hours.

As a summary, we can see that the impact of opportunistic networking applications when the mobile device sends information, is lower than merely remaining within an anchor zone. The Linked phase has the greatest power consumption, when the devices exchange information. Finally, the transmission data phase consumes less power because this action is intermittent, taking place only when there is an opportunity to establish a connection with another wireless device. 


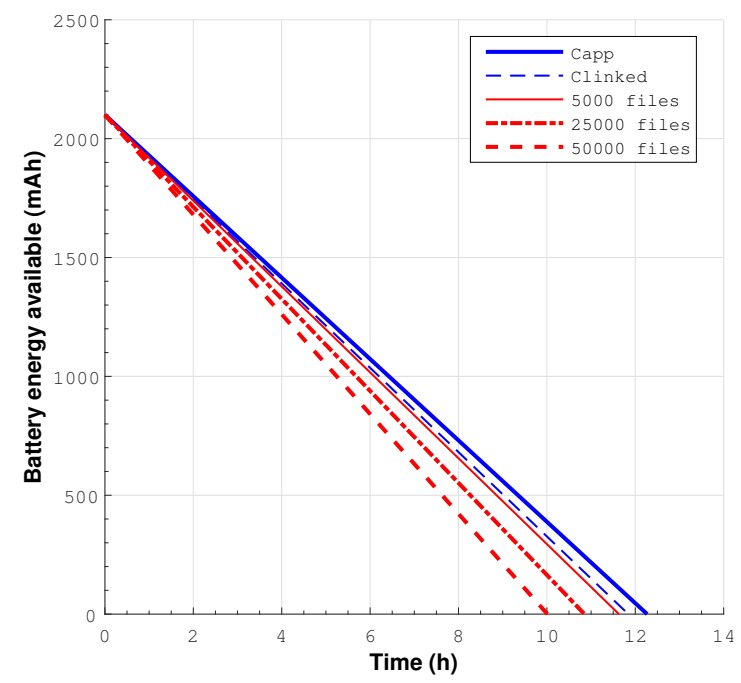

(a) Impact of data transmission.

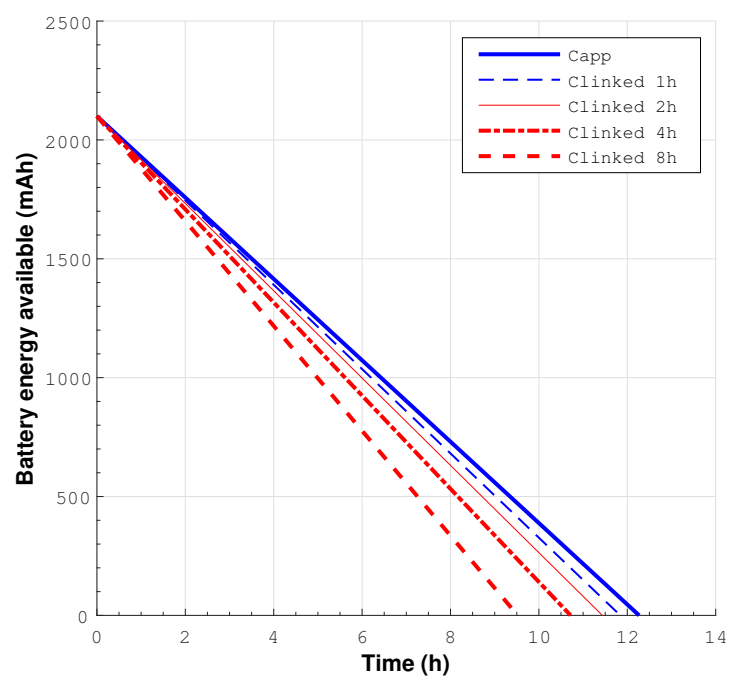

(b) Impact of the total Linked Time.

Figure 3.5: Impact on the battery performance. 


\subsection{Summary}

The main objective of this chapter was to perform power consumption measurements to study the impact of an application offering opportunistic networking. Based on the results, our evaluation showed that the power consumption in an opportunistic network is moderately low, making feasible and recommendable the deployment of applications of this communications environment. Furthermore, we conclude that, in opportunistic networking, the impact on mobile device batteries is greater since the application must be running permanently. An approach to reduce the battery consumption is to have the app running only when it is in an anchor zone. Also, we can state that, when a mobile device stays in the anchor zone, the power consumption is higher than when it is sending information. This is a consequence of the periodical swapping of protocol control information, a procedure required to receive and retransmit the packets in an anchor zone. Under these circumstances the correct and adequate tuning of this process is crucial to minimize the power consumption.

On the long term, our interest in this analysis is to increase the battery life by optimizing applications without losing their functionalities. Future works are focused on the following aspects: a) exploiting sensor information in the design and implementation of energy-efficient applications, and b) encouraging the collaboration between mobile devices by using of mobile apps designed with energy efficiency constraints. 



\section{Chapter 4}

\section{Buffer Management}

\subsection{Introduction}

In opportunistic networks the efficiency of message diffusion depends on the user's mobility, the transfer time, and the device buffer management. User's mobility determines the number of contacts and their duration while the transfer time depends on the size of the interchanged messages and the channel throughput. The device buffer management is a critical factor due to its limited size. The chosen policy for forwarding messages when a connection is available, and the message dropping policy when the buffer is full can impact greatly on the diffusion of the messages.

In this chapter, we analyze an opportunistic network solution from both pedestrian and vehicular perspective, taking into account the buffer management strategy to improve the epidemic message diffusion. We specifically focus on evaluating the impact of: (1) message sending priority, and (2) message discarding strategy. Our objective is to determine which approaches are optimal when selecting messages for sending, and for deleting messages when the buffer gets full.

By varying the buffer sizes and the messages TTL (Time To Live), we compared the performance of the basic Epidemic protocol in two scenarios (vehicular and pedestrian), using The ONE simulator.

The sending and receiving processes depend on the opportunity of contacting other devices, which is determined by node mobility. Therefore, the links between the devices are typically asymmetric and unstable because contacts are very dynamic.

The mobile nodes in a opportunistic network can be pedestrians or passengers 
in various types of vehicles (e.g., bus, car, taxi) carrying a mobile device (e.g., a smartphone). The duration of the contacts between the nodes is a key factor in the dissemination of messages; if the contact time is too short there will not be enough time for nodes to retrieve all pending messages. Therefore, the order in which messages are forwarded is a key factor to be considered. Moreover, the management details of the internal device buffer is also critical to provide an efficient service.

To obtain more realistic results we used two real mobility traces. These datasets are based on pedestrian traces experimentally obtained at the National Chengchi University (NCCU) campus [154], and the vehicular traces acquired by taxis in Rome [9]. The message generation patterns (frequency and size) are based on statistics related with social networking applications [135].

This chapter is organized in the following way: an overview of related works addressing opportunistic networks and message diffusion is presented in the next section. The description of our buffer management and diffusion proposals is provided in Section 4.3, while experiments and performance evaluation details are presented in Section 4.5. Finally, in Section 4.6, we present the section summary.

\subsection{Overview}

Considering the mobility factors such as speed, mobility model, node density, and places from a human environment, the authors of [105, 109, 123, 110], evaluate message dissemination in opportunistic networks. Experimental results obtained by simulation show that, as nodes start to move less (i.e., increasing pause time), less messages are expected to be delivered. Since nodes encounter at a low frequency rate, the number of message replications is also expected to decrease. Regarding latency, messages experience longer waiting times prior to delivery. On this context, the authors of [123], implemented a new forwarding protocol named HURRy protocol based on the PRoPHET routing mechanism. This routing is based on probabilistic techniques, although it incorporates the contact duration of encounters (unlike previous approaches) to estimate the rating probabilities of all possible paths to a certain destination.

In [58] the authors propose new approaches to improve the characterization of the inter-contact times distribution for real traces. Typical aggregate distributions are not representative of a single node behaviour because nodes are usually spread among several categories with highly distinct movement patterns. The approaches are validated with several trace examples, showing higher precision and representativeness than established aggregate distributions.

Regarding vehicular networks performance, the authors of $[26,177]$ performed large simulations using real traces to analyze the impact of different controllable and uncontrollable factors such as number of nodes, traffic load, packet TTL, transmission range, and propagation model. The results of this simulation process 
show that it is difficult to get good results in a real vehicular network due to its complexity. In [9], the authors evaluate the diffusion process using Epidemic protocols to determine the feasibility to implement opportunistic networks using vehicles as a mobile nodes in Rome, a city with irregular roads and traffic issues.

The hardware management of the mobile devices resources is considered as a key factor in the success of message delivery. In particular, one of the main constrains is memory capacity, known as buffer memory in the wireless networks context. For buffer management, several works $[40,42,117]$ evaluate the influence of the memory capacity on the delivery message ratio.

Finally, in [29] the authors make a extensive analysis of the storage in vehicular networks under the paradigm of floating content in opportunistic networks. In this work, we not only study the impact of buffer size but also different approaches for buffer management.

\subsection{Buffer Management Proposal}

In opportunistic networks both the node buffer size and the channel bandwidth are limited. It is therefore important to define mechanisms to optimize the use of these resources in a mobile device. Four different strategies are considered in this chapter (see Figure 4.1) for each of the two main buffer management activities (forwarding and dropping).

\section{Message Forwarding}

Message forwarding refers to the order for extracting messages from the buffer in order to transmit them to the connected device. We considered the four methods graphically described in Figure 4.1a. More specifically:

1. Random: The messages are selected randomly from those stored in the buffer. This method is already implemented in The ONE simulator and it is configured by default.

2. FIFO (First-In, First-Out): Messages are selected for forwarding according to their arrival order, i.e., the first message to be forwarded is the first message arriving at the mobile device (that is the oldest message, taking into account the reception time). From the figure: message $M 3$ arriving at time $t_{n}$ will be forwarded first, message $M 1$ arriving at time $t_{n+1}$ will be the next one to be sent, $M 1$ will be the following, and so on.

3. Oldest: With this approach, the message with lower TTL value will be forwarded first. In the example shown in the figure, M3 will be sent first, the next message will be $M 1$ with TTL value $t_{n+1}$, etc. (note, that this approach is different to the FIFO approach, as FIFO considers the arrival time of the message, while Oldest considers the TTL.) 


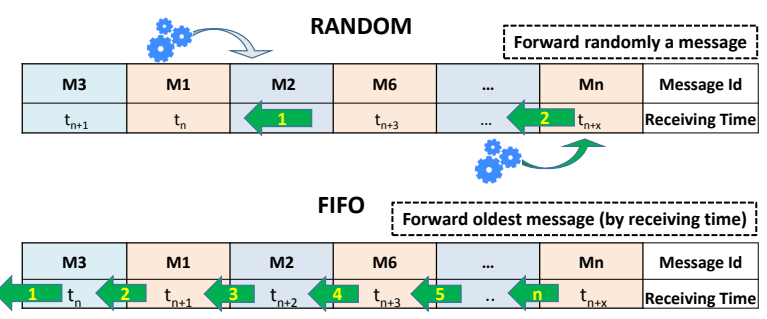

\begin{tabular}{|c|c|c|c|c|c|c|}
\hline \multirow[b]{2}{*}{ M3 } & \multirow[b]{2}{*}{ M1 } & \multicolumn{2}{|c|}{ OLDEST } & \multicolumn{3}{|c|}{ Forward First Oldest messages (< TTL) } \\
\hline & & M2 & M6 & $\ldots$ & $\mathrm{Mn}$ & Message Id \\
\hline & & $t_{n+2}$ & $t_{n+3}$ & & $t_{n+x}$ & TTL \\
\hline
\end{tabular}

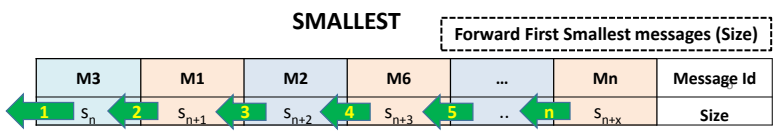

(a) Forwarding policies.
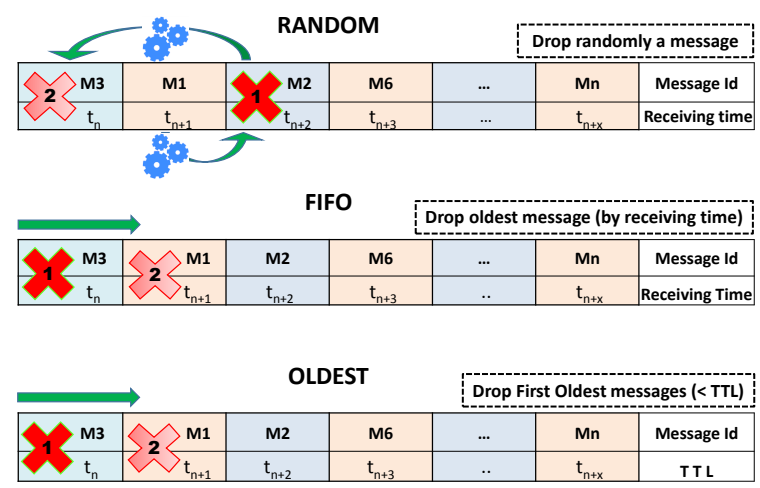

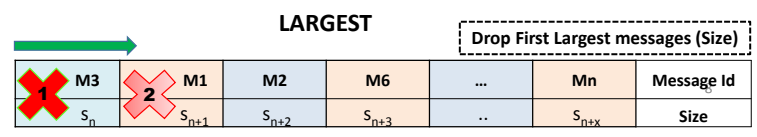

(b) Dropping policies.

Figure 4.1: Local buffer dropping and forwarding policies. 
4. Smallest: This approach aims at first sending the smallest messages. In the figure, we can see that the sending queue is organized according to the messages, size, i.e., $M 3$ with size $S_{n}$ will be forwarded first than message $M 1$ with a message size of $S_{n+1}$, since $S_{n}<S_{n+1}$.

On the original version, ONE only implements the Random and FIFO policies. The other two policies were implemented by the authors, as detailed in the following section.

\section{Message Dropping}

Message dropping refers to the order followed when making room for a new arriving message. For this, we need to determine which message or messages have to be dropped in order to make room for a new incoming message. This operation is usually executed when the buffer is full. Figure $4.1 \mathrm{~b}$ shows the considered approaches. More specifically:

1. Random: The message or messages to be removed from the buffer are selected randomly.

2. FIFO (First-In, First-Out): The first message to be removed from the buffer is the oldest message arriving at the mobile device, taking into account the reception time. From the figure: message $M 3$ that arrived at time $t_{n}$ will be removed first, and message $M 1$ with arrival time $t_{n+1}$ will be the next one, to be deleted.

3. Oldest: With this approach, the message with lower TTL value $t_{n}$ will be removed from the buffer. In the example in the Figure, $M 3$ will be the first, the next message to be dropped will be $M 1$ by having TTL value $t_{n+1}$, etc.

4. Largest: This approach removes the largest messages. In the figure, we can see that the dropping queue is organized according to the message size, i.e., M3 with size $S_{n}$ will be deleted first than message $M 1$ with a message size of $S_{n+1}$.

Since only the FIFO method was implemented in the ONE simulator, we implemented the other three policies modifying the ActiveRouter and MessageRouter. We added the ExponentialMessageGenerator to inject messages using an exponential distributed random interval. Finally, we added two subclasses of Report to obtain the buffer occupancy and transmission time information.

\subsection{Description of Experiments}

In order to evaluate the different buffer management approaches we employed The ONE simulator using two real movement traces of mobile users. The pedestrian 
trace (about 2.3 million of records) comes from an experiment at the National Chengchi University (NCCU) campus, where GPS position data was collected during two weeks (336 hours) using an Android app installed in the smartphones of 115 students in an area of $3.76 \mathrm{~km} \times 3.42 \mathrm{~km}$.

The vehicular trace (about 21 million of records) comes from a network formed by 370 taxi cabs in the vicinity of Rome during a whole month [141]. This GPS dataset was converted to Cartesian coordinates using the method described in [82] with an area covered of $100 \mathrm{~km} \times 100 \mathrm{~km}$ (complete dataset).

The workload considered tried to mimic the typical data-flow for a multimedia messaging application where shorter messages are far more common than larger ones. Three message sizes and frequencies were considered: (1) a short text message $(1 k B)$ every hour, $(2)$ a photo $(1 M B)$ every 18 hours, and $(3)$ a video or high resolution picture $(10 M B)$ every 96 hours. These frequencies were based on the statistics of WhatsApp message usage from [135], while sizes are approximations of the content produced by current mobile phone hardware. In order to obtain a realistic model of the user behaviour, the interval between messages is generated using an independent Poisson process for each user and message type.

The communication range $(r)$ in the case of pedestrian traces was set to $7.5 \mathrm{~m}$ with bandwidth $B_{w}=2.1 \mathrm{Mb} / \mathrm{s}$, and setup time $T_{s}=0.1 \mathrm{~s}$. These values are based on the Bluetooth 2.0 class 2 specifications, assuming some interferences that do not allow to obtain the full communication range $(10 \mathrm{~m})$. And, in the case of vehicular scenario, the general parameters are the same, except the communication range, where it is set to $50 \mathrm{~m}$, representing a WiFi interface configured in ad-hoc mode.

In order to compare the performance of the different buffer management policies, several simulations were executed for all the 16 possible combinations using the epidemic protocol for both traces. Each forwarding and dropping combination was tested with buffer sizes of 50MB, 100MB, 200MB and 1GB. These simulation parameters are summarized in Table 4.1.

Table 4.1: Simulation parameters to evaluate queue management policies.

\begin{tabular}{|c|c|}
\hline Parameter & Values \\
\hline \hline Buffer Size & $50 \mathrm{MB}, 100 \mathrm{MB}, 200 \mathrm{MB}, 1 \mathrm{~GB}$ \\
\hline Message size & $1 \mathrm{kB}, 1 \mathrm{MB}, 10 \mathrm{MB}$ \\
\hline Routing & Epidemic \\
\hline Time to Live & 12 hours, 24 hours \\
\hline Forward policy & Random, FIFO, Oldest, Smallest \\
\hline Discard policy & Random, FIFO, Oldest, Largest \\
\hline
\end{tabular}




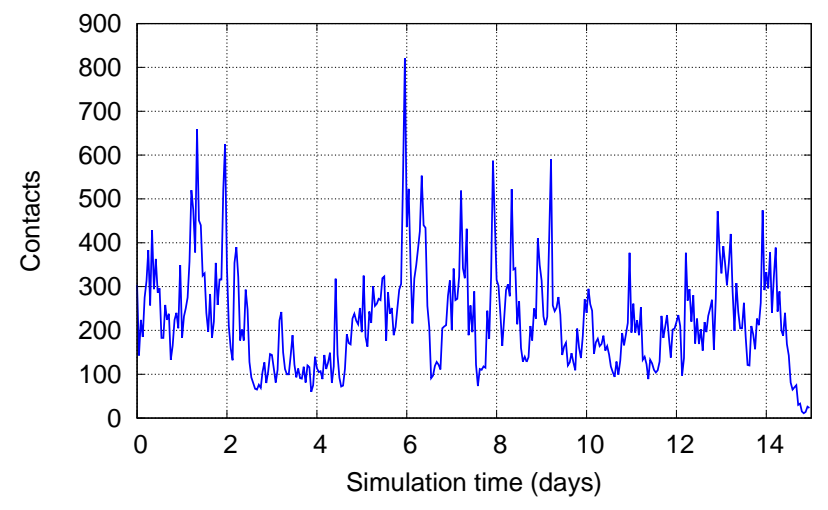

(a) Pedestrian trace

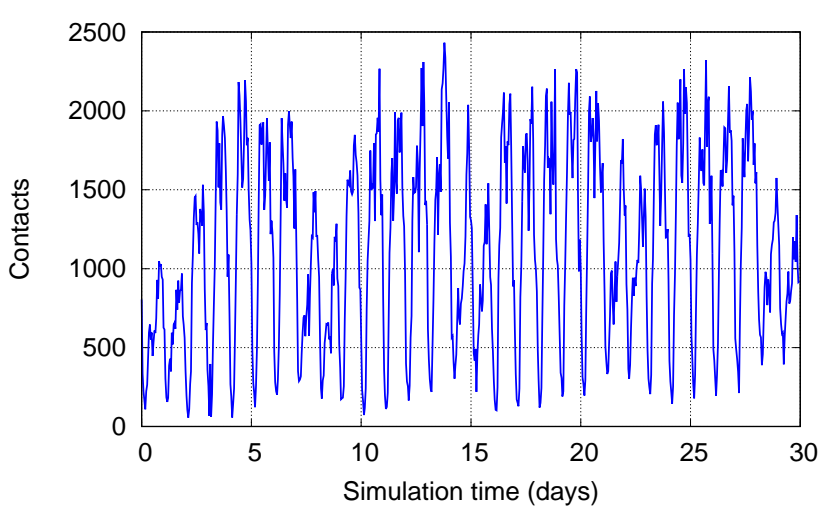

(b) Vehicular trace

Figure 4.2: Number of contacts at each simulation hour.

\subsection{Performance Evaluation}

The average inter-contact time (defined as inter-any-contact time in [58]) in the NCCU traces is 16 seconds and the average contact duration is 3 seconds. In the Roma taxi traces those averages are respectively 4.25 and 3.2 seconds. However, those averages are not very representative of the contact distribution through time, which has a clear periodic behaviour as shown in Figure 4.2. Each point in these plots corresponds to the number of contacts during each hour of the simulation. The daily contact pattern is clearly visible in the vehicular trace, even a weekend versus workday cycle can be observed. The daily cycle is not that clear in the pedestrian trace but it is still present. Taking into account this observation we 
decide to run two sets of simulations using a message TTL of 12 and 24 hours.

Tables 4.2 , and 4.3 show the delivery probability for each trace, and the TTL and policy combination considered. The vehicular trace obtains a higher delivery probability (delivered_messages/created_messages) than the pedestrian simply because it has more contacts and they last longer. A TTL of 24 hours allows to keep the messages in the buffers enough time for them to be delivered during the whole daily cycle thus greatly improving the delivery probability. As expected, a bigger buffer also improves the delivery probability, in particular when combined with a 24 hours TTL. With a 1 GB buffer in the $12 \mathrm{~h}$ and $24 \mathrm{~h}$ cases and $200 \mathrm{MB}$ in the $12 \mathrm{~h}$ TTL case the dropping policy is no longer relevant because the buffer is sufficiently large to keep all the alive messages. In all cases the best buffer management is the combination of smallest forwarding and largest dropping policies because they give priority to small messages which have a better transmission chance with short contacts.

Figures 4.3 and 4.4 show the relationship between delivery probability and average message latency for each of the simulations. This plot uses a point for each buffer management policy combination with the same symbol for a particular buffer size. The plot lines show the correlation between delivery probability and latency for each buffer size. In all cases a large probability implies a short latency. When the buffer size increases this correlation is even higher and the variability of the delivery probability decreases across the different policies. For a TTL of 12 hours, both traces show different performance as expected from their different contact properties, with a larger delivery probability and lower latency for the vehicular trace. However, with a TTL of 24 hours both traces are closer in performance, suggesting that the pedestrian trace somehow achieves better connectivity during each daily cycle.

Considering protocol overhead, Figure 4.5a shows the maximum of the average buffer occupancy from each node. With the simulated workload a buffer of around 80 and $175 \mathrm{MB}$ seems sufficient for a TTL of 12 and 24 hours respectively.

In Epidemic protocol, another overhead measurement is the amount of information forwarded per node. Figure 4.5b shows the daily average of bytes forwarded by each node (that is, the sum of all bytes transmitted divided by the number of nodes and the simulation length in days). The amount of transmissions increases greatly with a larger buffer size and TTL time, basically since more messages stay in the buffers during more time. We consider that this is a critical result from the energy usage point of view indicating that a strategy to inform nodes about the actual reception of the messages would be necessary to avoid useless retransmissions. 
Table 4.2: Delivery probability for different TTL, buffer sizes, and queue policies using the pedestrian trace. In bold type are the best results for each combination.

(a) TTL 12 hours.

\begin{tabular}{|c|c|r|r|r|r|}
\hline \multirow{2}{*}{$\begin{array}{c}\text { Buffer } \\
\text { size }\end{array}$} & $\begin{array}{c}\text { Drop } \\
\text { policy }\end{array}$ & \multicolumn{4}{|c|}{ Forwarding policy } \\
\cline { 3 - 6 } & Random & FIFO & TTL & Smallest \\
\hline \hline \multirow{4}{*}{$50 \mathrm{MB}$} & Random & 0.394 & 0.386 & 0.380 & 0.415 \\
\cline { 2 - 6 } & FIFO & 0.403 & 0.398 & 0.395 & 0.415 \\
\cline { 2 - 6 } & TTL & 0.393 & 0.377 & 0.366 & 0.417 \\
\cline { 2 - 6 } & Largest & 0.433 & 0.425 & 0.421 & $\mathbf{0 . 4 4 0}$ \\
\hline \multirow{4}{*}{$100 \mathrm{MB}$} & Random & 0.426 & 0.419 & 0.415 & 0.439 \\
\cline { 2 - 6 } & FIFO & 0.427 & 0.420 & 0.417 & 0.438 \\
\cline { 2 - 6 } & TTL & 0.426 & 0.418 & 0.415 & 0.439 \\
\cline { 2 - 6 } & Largest & 0.433 & 0.424 & 0.420 & $\mathbf{0 . 4 4 2}$ \\
\hline $200 \mathrm{MB}$ & Any & 0.434 & 0.427 & 0.423 & $\mathbf{0 . 4 4 4}$ \\
\hline $1 \mathrm{~GB}$ & Any & 0.434 & 0.427 & 0.423 & $\mathbf{0 . 4 4 4}$ \\
\hline
\end{tabular}

(b) TTL 24 hours.

\begin{tabular}{|c|c|r|r|r|r|}
\hline \multirow{2}{*}{$\begin{array}{c}\text { Buffer } \\
\text { size }\end{array}$} & $\begin{array}{c}\text { Drop } \\
\text { policy }\end{array}$ & \multicolumn{4}{|c|}{ Forwarding policy } \\
\cline { 3 - 6 } & Random & FIFO & TTL & Smallest \\
\hline \hline \multirow{4}{*}{$50 \mathrm{MB}$} & Random & 0.473 & 0.449 & 0.442 & 0.559 \\
\cline { 2 - 6 } & FIFO & 0.505 & 0.496 & 0.490 & 0.565 \\
\cline { 2 - 6 } & TTL & 0.472 & 0.445 & 0.440 & 0.582 \\
\cline { 2 - 6 } & Largest & 0.625 & 0.600 & 0.600 & $\mathbf{0 . 6 3 8}$ \\
\hline \multirow{4}{*}{$100 \mathrm{MB}$} & Random & 0.538 & 0.519 & 0.519 & 0.607 \\
\cline { 2 - 6 } & FIFO & 0.557 & 0.548 & 0.535 & 0.618 \\
\cline { 2 - 6 } & TTL & 0.558 & 0.544 & 0.533 & 0.621 \\
\cline { 2 - 6 } & Largest & 0.634 & 0.619 & 0.610 & $\mathbf{0 . 6 4 5}$ \\
\hline & Random & 0.609 & 0.603 & 0.604 & 0.643 \\
\cline { 2 - 6 } & FIFO & 0.616 & 0.607 & 0.604 & 0.643 \\
\cline { 2 - 6 } & TTL & 0.619 & 0.608 & 0.607 & 0.647 \\
\cline { 2 - 6 } & Largest & 0.632 & 0.616 & 0.612 & $\mathbf{0 . 6 4 9}$ \\
\hline $1 \mathrm{~GB}$ & Any & 0.635 & 0.625 & 0.625 & $\mathbf{0 . 6 5 2}$ \\
\hline
\end{tabular}


Table 4.3: Delivery probability for different TTL, buffer sizes, and queue policies using the vehicular trace.

(a) TTL 12 hours.

\begin{tabular}{|c|c|r|r|r|r|}
\hline \multirow{2}{*}{$\begin{array}{c}\text { Buffer } \\
\text { size }\end{array}$} & $\begin{array}{c}\text { Drop } \\
\text { policy }\end{array}$ & \multicolumn{4}{|c|}{ Forwarding policy } \\
\cline { 3 - 6 } & Random & FIFO & TTL & Smallest \\
\hline \hline \multirow{4}{*}{$50 \mathrm{MB}$} & Random & 0.436 & 0.407 & 0.410 & 0.507 \\
\cline { 2 - 6 } & FIFO & 0.449 & 0.429 & 0.424 & 0.503 \\
\cline { 2 - 6 } & TTL & 0.434 & 0.410 & 0.407 & 0.511 \\
\cline { 2 - 6 } & Largest & 0.497 & 0.475 & 0.466 & $\mathbf{0 . 5 3 0}$ \\
\hline \multirow{3}{*}{$100 \mathrm{MB}$} & Random & 0.476 & 0.450 & 0.449 & 0.528 \\
\cline { 2 - 6 } & FIFO & 0.479 & 0.459 & 0.452 & 0.527 \\
\cline { 2 - 6 } & TTL & 0.479 & 0.454 & 0.449 & 0.530 \\
\cline { 2 - 6 } & Largest & 0.508 & 0.484 & 0.468 & $\mathbf{0 . 5 3 5}$ \\
\hline $200 \mathrm{MB}$ & Any & 0.505 & 0.487 & 0.475 & $\mathbf{0 . 5 3 7}$ \\
\hline $1 \mathrm{~GB}$ & Any & 0.509 & 0.490 & 0.476 & $\mathbf{0 . 5 3 8}$ \\
\hline
\end{tabular}

(b) TTL 24 hours.

\begin{tabular}{|c|c|r|r|r|r|}
\hline \multirow{2}{*}{$\begin{array}{c}\text { Buffer } \\
\text { size }\end{array}$} & $\begin{array}{c}\text { Drop } \\
\text { policy }\end{array}$ & \multicolumn{4}{|c|}{ Forwarding policy } \\
\cline { 2 - 6 } & Random & FIFO & TTL & Smallest \\
\hline \hline \multirow{4}{*}{$50 \mathrm{MB}$} & Random & 0.556 & 0.511 & 0.509 & 0.665 \\
\cline { 2 - 6 } & FIFO & 0.571 & 0.544 & 0.534 & 0.656 \\
\cline { 2 - 6 } & TTL & 0.534 & 0.525 & 0.525 & 0.671 \\
\cline { 2 - 6 } & Largest & 0.678 & 0.657 & 0.627 & $\mathbf{0 . 7 0 3}$ \\
\hline \multirow{4}{*}{$100 \mathrm{MB}$} & Random & 0.618 & 0.586 & 0.577 & 0.695 \\
\cline { 2 - 6 } & FIFO & 0.628 & 0.606 & 0.588 & 0.694 \\
\cline { 2 - 6 } & TTL & 0.628 & 0.619 & 0.596 & 0.698 \\
\cline { 2 - 6 } & Largest & 0.683 & 0.667 & 0.644 & $\mathbf{0 . 7 1 0}$ \\
\hline \multirow{3}{*}{$200 \mathrm{MB}$} & Random & 0.664 & 0.646 & 0.633 & 0.710 \\
\cline { 2 - 6 } & FIFO & 0.667 & 0.653 & 0.635 & 0.709 \\
\cline { 2 - 6 } & TTL & 0.669 & 0.657 & 0.638 & 0.711 \\
\cline { 2 - 6 } & Largest & 0.688 & 0.671 & 0.649 & $\mathbf{0 . 7 1 4}$ \\
\hline $1 \mathrm{~GB}$ & Any & 0.693 & 0.682 & 0.660 & $\mathbf{0 . 7 1 7}$ \\
\hline
\end{tabular}




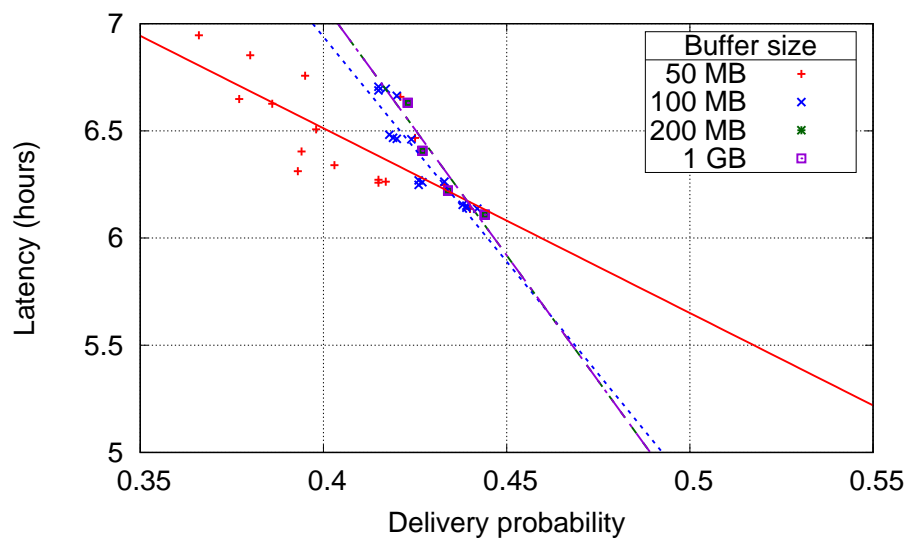

(a) 12 hours case.

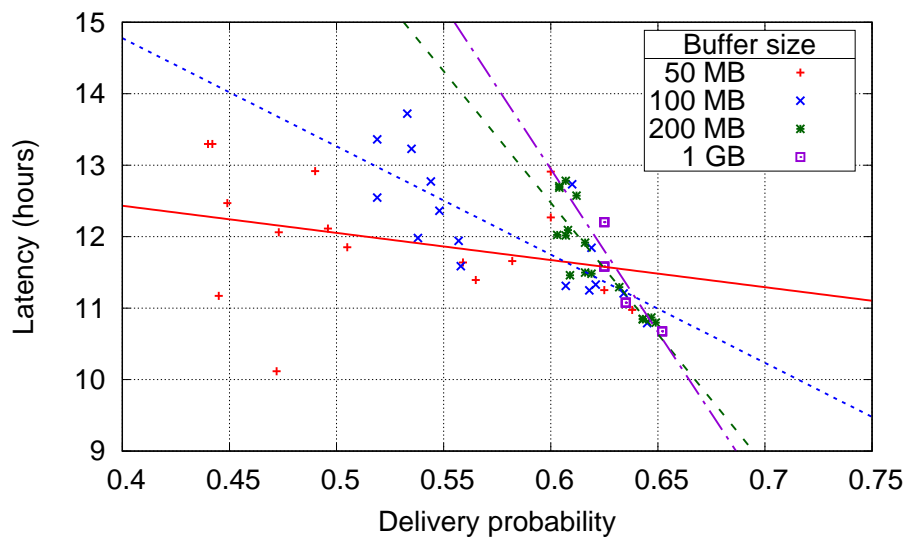

(b) 24 hours case.

Figure 4.3: Delivery probability versus latency for a 12 and 24 hours TTL with different buffer sizes and queue policies using the pedestrian trace. 


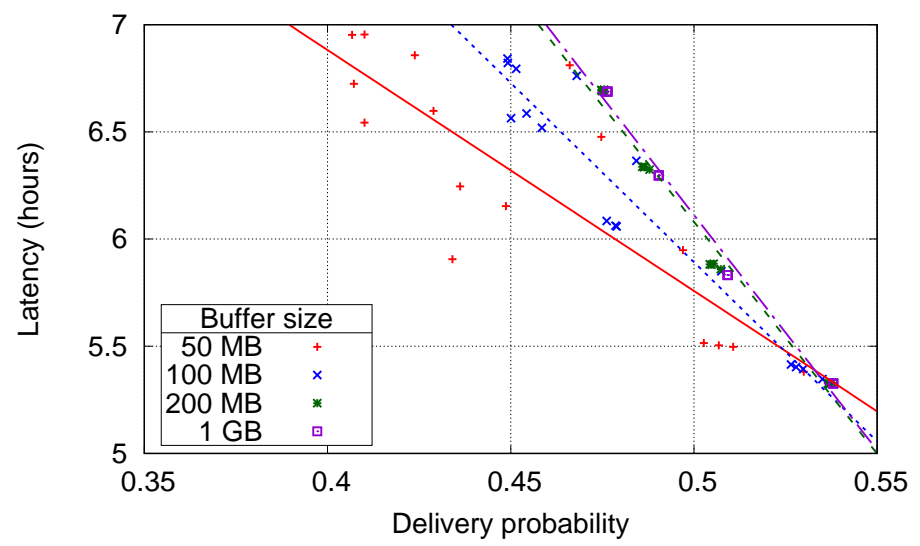

(a) 12 hours case.

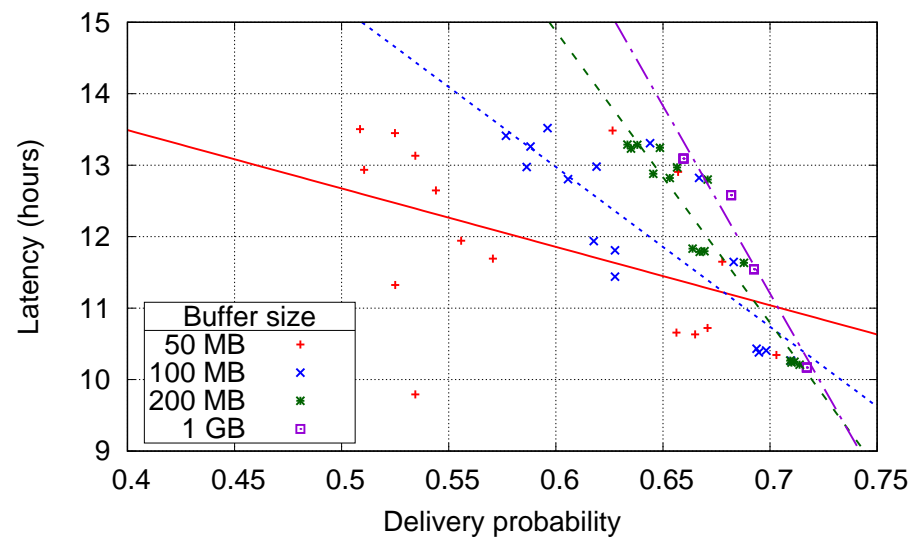

(b) 24 hours case.

Figure 4.4: Delivery probability versus latency for a 12 and 24 hours TTL with different buffer sizes and queue policies using the vehicular trace. 


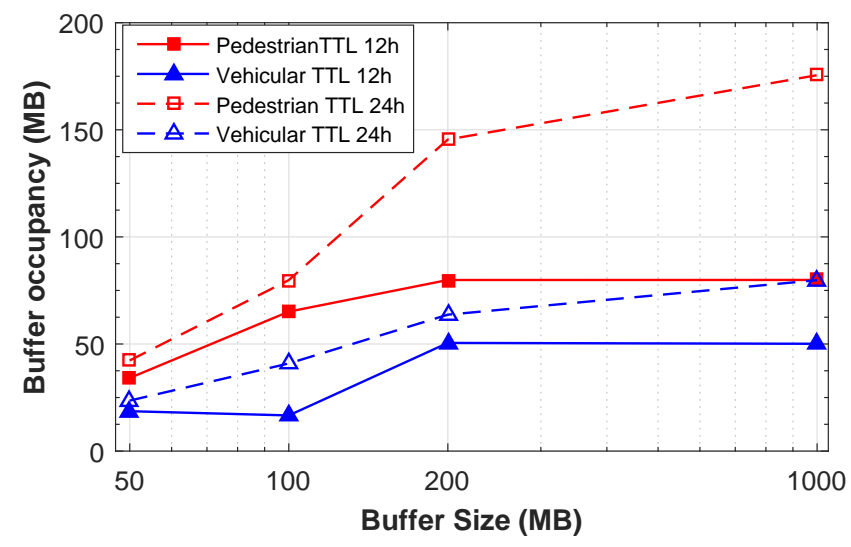

(a) Maximum of the average buffer occupancy from each node.

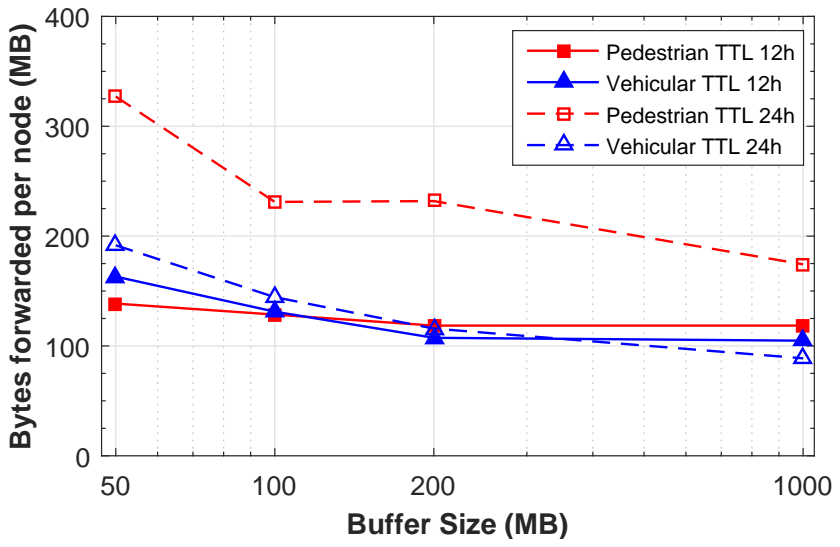

(b) Average bytes daily forwarded per node (y-axis in log scale).

Figure 4.5: Overhead results: buffer occupancy and forwarded bytes 


\subsection{Summary}

In this chapter we evaluated the impact of different buffer management strategies on multimedia messaging applications for opportunistic wireless networks. We showed that, the greater the number of messages in local buffers, the better the diffusion is (in terms of delivery probability and latency). To keep a large number of messages not only a large buffer size is relevant but also the adequate buffer management policy. The best performance is achieved when forwarding the smallest messages first and dropping the biggest ones to make room for incoming transmissions.

The experiments show a clear trade-off between delivery probability and latency. Approximately, doubling the TTL from 12 to 24 hours improves the delivery probability by $50 \%$, but the latency is also doubled. This limit on the delivery performance of opportunistic networks depends on the devices movement, which affects the number and duration of contacts. Although the two considered traces came from very different origins, the node behaviour is quite similar, converging together at periodic intervals (daily) to a well defined set of places (classrooms, taxi stops). Therefore, a TTL of 24 hours greatly improves delivery probability because allows messages to be transmitted for the whole daily period.

Regarding buffer size, we found that, with the simulated workload, simply $175 \mathrm{MB}$ are sufficient even for a TTL 24 hours. The drawback of such a large TTL time in the Epidemic protocol is the huge amount of useless transmissions, which drain the already scarce battery power of mobile devices. It would be interesting to design strategies to inform nodes about the actual message receptions and avoid unnecessary retransmissions. 


\section{Chapter 5}

\section{Use of Long Range Technologies}

\subsection{Introduction}

The message delivery ratio of mobile opportunistic networks strongly depends on the available transmission time, which is closely related to the mobility of users and to the communication properties of the mobile devices. A larger radio transmission range allows longer contact duration, improving the message dissemination. Furthermore, user mobility is a crucial factor to be considered, especially when the mobile nodes are in vehicles, because of their limited freedom of movement and the high relative speed.

In this chapter, we evaluate the use of a sub-gigahertz wireless technology, namely LoRa (Long Range), to establish links between the mobile users in an opportunistic network in order to augment the number of contacts and their duration. We evaluate the performance of LoRa, comparing it with WiFi, using the Epidemic protocol for message diffusion with realistic vehicular traces. Through simulations, we compare the message delivery probability and the network overhead. These experiments were carried out using The ONE simulator with some minor modifications to model the typical behaviour of mobile users. The results show that, in opportunistic networks, increasing the range even at the cost of reducing the available bandwidth increases the message delivery ratio.

The outline of the chapter is as follows: an overview of related works about opportunistic vehicular networks and message diffusion is presented in Section 5.2, a LoRa test bed platform is presented in Section 5.3, and the experiments evaluation is presented in Section 5.5. Finally, Section 5.6 contains some conclusions and future work. 


\subsection{Overview}

Vehicular Delay Tolerant Networks (VDTNs) [35, 87, 7, 159] have been proposed as a novel strategy to provide data transmission in vehicular scenarios. One type of networks inside the VDTN model are the Vehicular Opportunistic Networks. Other authors, such the ones in $[112,44]$, define them as a subclass of DTNs. The reference communication model is typically based on the Epidemic protocol [157]. This protocol is widely used as a reference technique and its operations are based on the store, carry, and forward approach combined with the flooding of messages.

In the same context using another trace set of 4,000 taxis, the author of [99] validated the collected data, and created their own mobility model called Shanghai Urban Vehicular Network (SUVnet).

In $[9,14,48]$ the authors examine the performance of protocols in opportunistic networks considering GPS information of large cities, like Rome, Berlin, Beijing, among others. In $[101,27]$ the authors propose improvements to the diffusion protocols using analytical models tested by simulations. In the context of VANETs, the authors of [153] extend the Internet connection between cars using embedded devices such as Raspberry Pi.

POR is proposed in [66] as a new Opportunistic Routing (OR) protocol for high-speed, multi-rate wireless mesh networks that runs on commodity WiFi interfaces supporting TCP. Its performance is analyzed with a testbed with 16 fixed nodes in a mesh distribution, showing improvements to data transfer. A similar idea [94] is used to face the problem of high speed vehicles proposing a two-way routing protocol extending the access point connectivity through opportunistic routing. Also they demonstrate how to exploit the navigation system to predict mobility and route messages.

The above listed works propose performance improvements for the Epidemic dissemination of messages, taking into account different aspects of vehicular networks. Most of these proposals have been tested through simulations and testbeds, however none of them considered the use of sub-gigahertz wireless technologies with longer range to improve the message diffusion.

In this chapter is evaluated, through simulations, the performance of the Epidemic protocol in a vehicular opportunistic network when employing two different data transmission technologies: WiFi (more exactly WiFi-Direct) and the novel LoRa (Long Range). The latter provides greater communication range by working at sub-gigahertz frequencies, thus generating more contacts with longer duration, but provides a reduced bandwidth when compared to the former. Also, it is evaluated the impact of both technologies in terms of ratio message delivery, latency, buffer consumption, and the contact duration time for different buffer sizes and message TTLs (Time To Live). The ONE simulator is parametrized with real GPS vehicular traces acquired from [18] while the frequency and size of messages are based on social networking applications [135]. 
1)

Raspberry Pi
2)

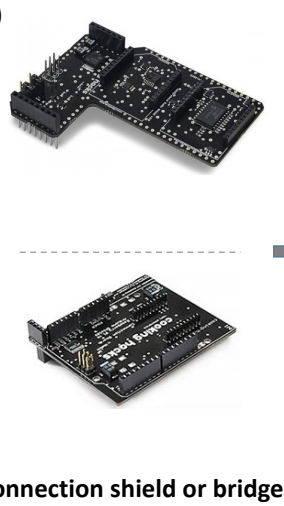

Connection shield or bridge
3)
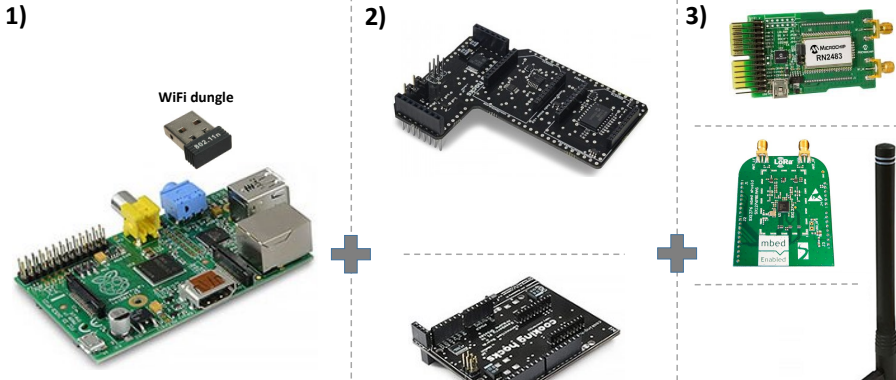

3)

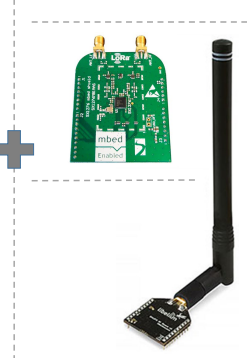

LoRa chip

(a) Network components.
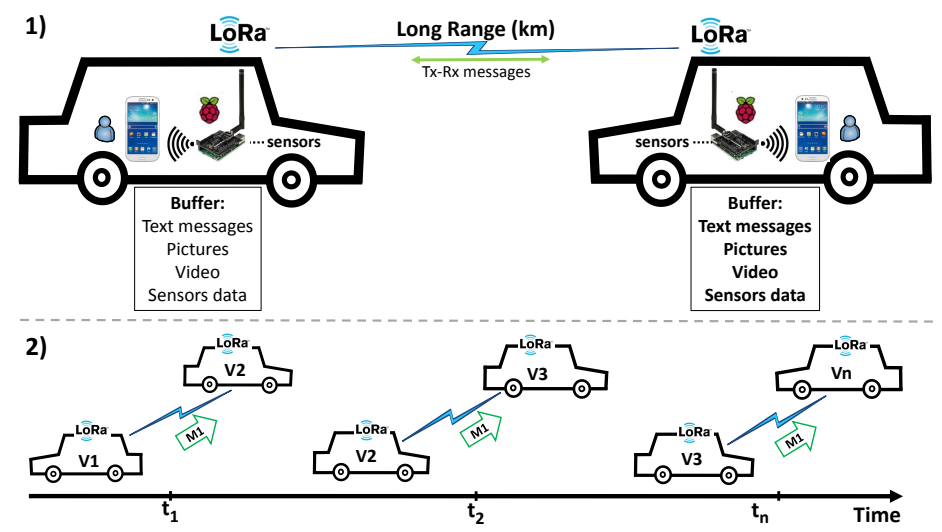

(b) Diffusion among mobile nodes.

Figure 5.1: Vehicular opportunistic network components and diffusion scheme. 


\subsection{Long-Range Data Transmission Proposal}

In this section we describe some details of a possible data transmission system based on LoRa by depicting an architecture aimed to provide an opportunistic communication module for vehicular nodes.

\subsubsection{Message Diffusion Design}

In this subsection we present a possible design for an opportunistic message transmission device for vehicular networks using LoRa. Figure 5.1 shows the components and their interactions to implement an opportunistic communication system. Figure 5.1a depicts the hardware elements: 1) a Raspberry Pi device with a WiFi dongle, 2) one connection bridge or shield, and 3) a LoRa interface connected to the Raspberry Pi via the connection bridge. These components together will allow general WiFi devices (e.g., smartphones) to communicate through the LoRa interfaces using the Raspberry Pi as a bridge. It is important to note the frequency restrictions depending on each country, e.g. in Europe LoRa is authorized to use the bands of $433 \mathrm{MHz}$ and $868 \mathrm{MHz}$, and in USA 915Mhz.

On the top part of Figure 5.1b, we illustrate the interaction between devices that are embedded on the vehicles. On the bottom part of this figure we also show an example of the Epidemic diffusion scheme, where a vehicle $V_{1}$ transmits the message $M_{1}$ to $V_{2}$, after some time $V_{2}$ sends the message to $V_{3}$ when both vehicles are in communication range, and the process continues until the message arrives to its destination.

\subsection{Description of Experiments}

In order to evaluate the feasibility of our proposal we employed The ONE simulator using a real vehicular movement trace and generating a network load based on typical multimedia mobile messaging applications.

Table 5.1: Simulation parameters varied to evaluate message diffusion.

\begin{tabular}{|c|c|}
\hline Parameter & Values \\
\hline \hline Buffer Size & $50 M B, 100 M B, 200 M B, 1 G B$ \\
\hline Routing & Epidemic \\
\hline Mobile nodes & 316 \\
\hline Time to Live & 6 hours, 12 hours \\
\hline Bandwidth & $2 M b / s$ (WiFi-Direct), $50 \mathrm{~Kb} / \mathrm{s}$ (LoRa) \\
\hline Tx-Range & $50 \mathrm{~m}$ (WiFi-Direct), $2500 \mathrm{~m}$ (LoRa) \\
\hline
\end{tabular}


The vehicular trace (about 21 million of records) comes from a network formed by 316 taxi cabs in the vicinity of Rome during a whole month [18]. This GPS dataset was converted to Cartesian coordinates using a traverse Mercator projection [82] centred near the Coliseum covering an area of $100 \mathrm{~km} \times 100 \mathrm{~km}$. Figure 5.2 shows how the vehicular traces are distributed around the metropolitan area. Figure $5.2 \mathrm{~b}$ is a zoomed view of the previous one, showing how the main urban area is almost fully covered by the traces.

The workload considered is the same that in the previous section 4.4. Where three message sizes and frequencies were considered: (1) a short text message $(1 \mathrm{kB})$ every hour, $(2)$ a photo $(1 \mathrm{MB})$ every 18 hours, and (3) a video or highresolution picture (10MB) every 96 hours.

For our experiments we modified The ONE simulator. Concretely, The ONE message generator (the MessageEventGenerator class), that injects a new message using a random interval time. This random time is uniformly distributed from a range configured in the simulation parameters. In order to obtain a more realistic model, we implemented an independent Poisson process for each user and message type, using an exponential distributed random generator.

\subsection{Performance Evaluation}

In order to compare the diffusion performance with both wireless technologies we run a battery of simulations varying the communication range and transmission bandwidth. WiFi-Direct is simulated with a range of $50 \mathrm{~m}$ and a bandwidth of $2.1 \mathrm{Mb} / \mathrm{s}$ while LoRa parameters are $2,500 \mathrm{~m}$ and $50 \mathrm{~kb} / \mathrm{s}$ respectively (values based on the LoRa class B specifications).

Beside transmission range and speed, we also varied several key parameters such as buffer size and message TTL. The effect of these parameters has been analyzed in our previous research [62]. In order to keep the number of simulations under reasonable limits, we only test the buffer management policy that has shown the best performance: prioritize small messages for transmission and large message for dropping when the buffer is full. Table 5.1 summarizes the main parameters and the different values used in the performed experiments.

Figure 5.3 shows the number of contacts generated during the simulation among the taxis for both transmission technologies. As expected, the large range of LoRa greatly increases contacts (up to 10 times). Furthermore, the average contact time increases from about two minutes with WiFi to 34 using LoRa, and the average inter-contact time (defined as the inter-any-contact time in [58]) with LoRa is about 7 times shorter.

Figure 5.4a shows the average delivery success ratio (i.e. delivery probability) for both technologies varying buffer size and TTL. This ratio is computed as the quotient between the number of messages that reach their destination and the number of messages generated in the simulation. This plot shows clearly the 


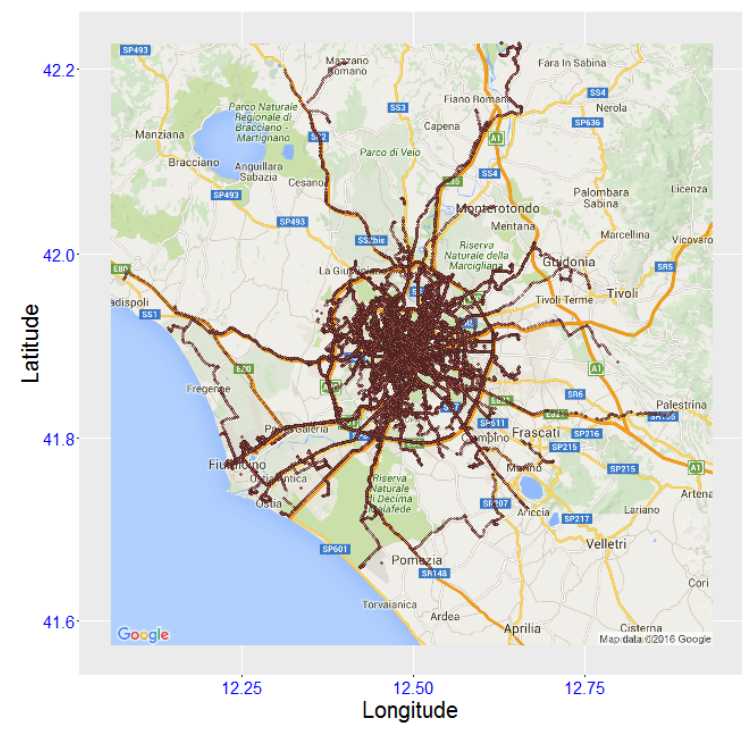

(a) Metropolitan area of Rome (Zoom 10).

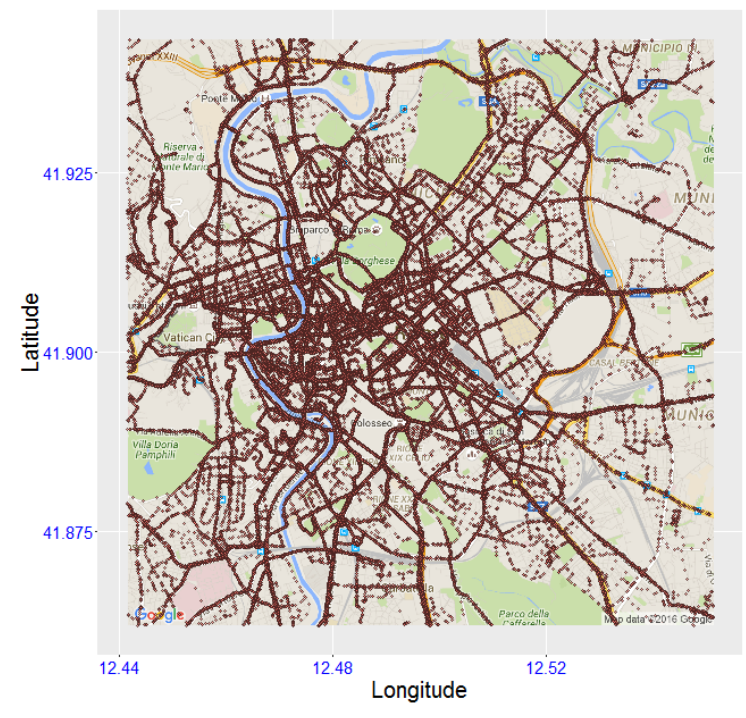

(b) Rome city center (Zoom 13)

Figure 5.2: Rome trace sample of $6 \mathrm{~h}$ of GPS traces. Total length of trace is 30 days, 316 taxis in an area of $10,000 \mathrm{~km}^{2}\left(0.0316\right.$ nodes per $\left.\mathrm{km}^{2}\right)$. 


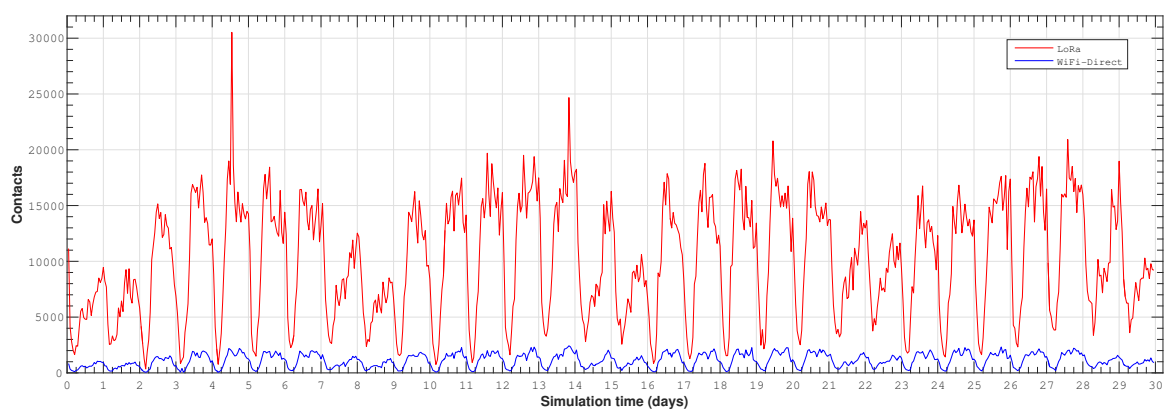

Figure 5.3: Number of contacts per hour generated by the simulation for each transmission technology.

huge advantage (up to 50\%) of LoRa over WiFi thanks to the larger number of contacts. We can also see that a larger TTL improves the Epidemic diffusion for both technologies, but in the LoRa case, this improvement is not as significant as in WiFi. Furthermore, the influence of buffer size is negligible for this workload.

Figure 5.4b plots the average latency for all messages varying TTL and buffer size for both technologies. As in the previous figure the advantage of LoRa is clear (up to $40 \%$ ) while the impact of the TTL is not as important as for WiFi and buffer size is also not relevant.

Latency shows an inverse relationship to delivery probability, typical of the Epidemic diffusion process. Allowing messages to stay longer, increases their probability to be delivered in a future contact but this delivery will also have an increased latency. That is, more messages reach the destination, but with greater latency. These experiments show a clear trade-off between delivery probability and latency. Allowing a large TTL improves greatly the delivery probability but also increases the latency.

The main drawback of the Epidemic diffusion process is the large overhead both in buffer occupation and bytes transmitted. Figures 5.5a and 5.5b show both results for the simulations performed. We can see that a buffer of $200 \mathrm{MB}$ is large enough to keep all messages generated and it seems that a small buffer could get almost full. However this does not affect message delivery in a significant way, as shown in Figure 5.4a. The amount of bytes forwarded with LoRa is similar for the different buffer sizes and TTL parameters. However, with WiFi this amount is larger for small buffer sizes, this is a side effect of the epidemic process. When two nodes establish a contact and one of them has a full buffer, messages are sent and discarded in a loop until the contact breaks, increasing artificially the amount of bytes forwarded. This effect is not as important with LoRa because of the very low bandwidth. 


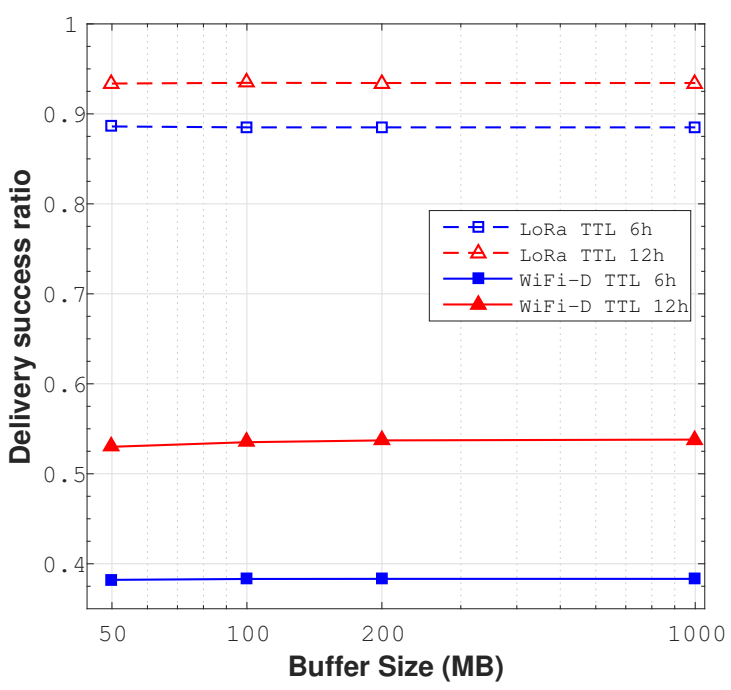

(a) Delivery probability.

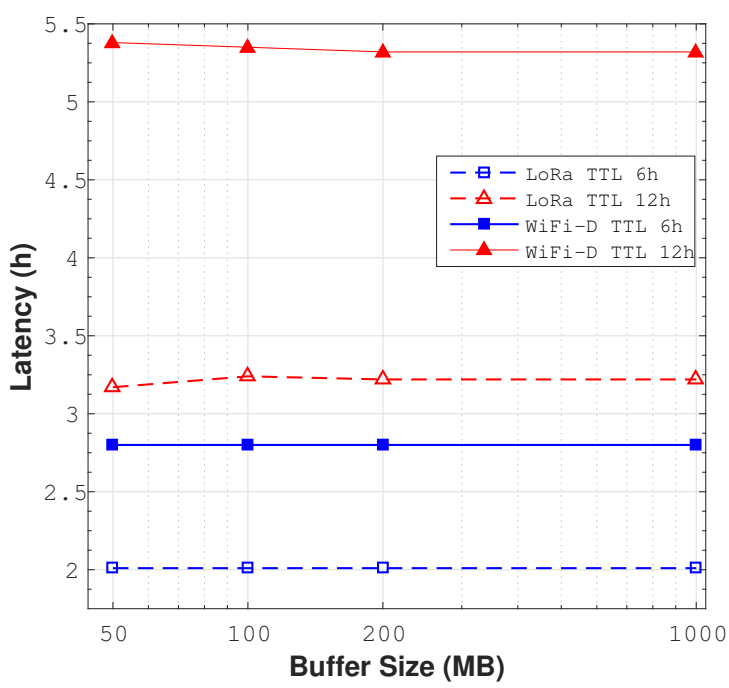

(b) Latency time (hours).

Figure 5.4: Average delivery success ratio and latency. 


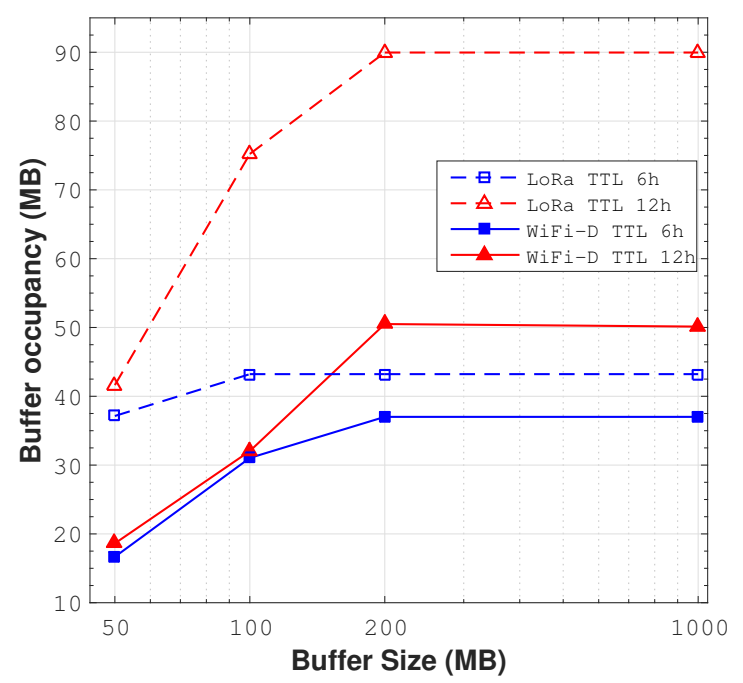

(a) Maximum of the average buffer occupancy from each node.

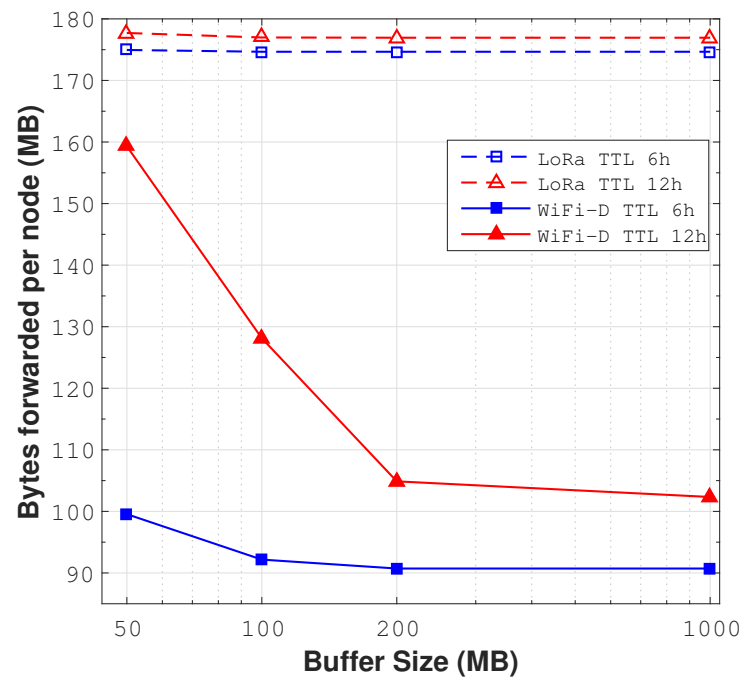

(b) Average bytes daily forwarded per node.

Figure 5.5: Overhead results: Buffer occupancy and forwarded bytes 


\subsection{Summary}

In this chapter we evaluated the impact of a sub-gigahertz wireless technology, in our case the novel Long Range (LoRa) technology, in a opportunistic network using the Epidemic protocol. The presented simulations were based on a real world movement trace from taxis of Rome and a workload from typical multimedia message applications. Two different scenarios were compared: one with short-range/high-bandwidth (WiFi), and another with long-range/low-bandwidth (LoRa).

In the studied scenario, LoRa improves significantly the message delivery ratio over $\mathrm{WiFi}$ in the range of about $40 \%$ to $50 \%$ for TTLs of 12 and 6 hours respectively. This is because a wider communication range allows not only more contacts but also those contacts will have longer durations. As we can see, in opportunistic networks, the delivery ratio is limited by the number of contacts so the communication range becomes the most important factor after message TTL or buffer size, leaving the available bandwidth as a no-crucial factor. 


\section{Chapter 6}

\section{Inclusion of Fixed Nodes}

\subsection{Introduction}

The use of opportunistic communications to push common interest messages in an urban scenario can possibly have various applications, e.g., sharing traffic status, advertising shop offers, spread alarms, and so on. In this chapter, we evaluate the combined use of fixed and mobile nodes to establish an urban opportunistic network aimed at the distribution of general interest data. We consider two very large realistic vehicular traces belonging to cities with different street organizations, namely Rome and San Francisco. Through simulations, we compare the message delivery probability and the network overhead. These experiments were carried out using two different simulators - The ONE and OMNeT ++ . The experiments design and its executions were done together with the Sustainable Communication Networks research group (Comnets) at the University of BremenGermany, during the author's internship in 2016.

Our results not only contradict current assumptions about the combination of fixed and mobile nodes, but also led us to some very interesting general-purpose observations about the dynamics of opportunistic networks. For example we found that mobility is not the hindering and challenging property of these networks, but probably their main enabler. Moreover, we determined that if we want to increase the performance of opportunistic networks by increasing the node density, we should add mobile and not fixed nodes. Adding fixed nodes only increases the overhead.

The outline of the chapter is as follows: an overview of related works about opportunistic vehicular networks and message diffusion is presented in Section 
6.2, our experimental scenarios are described in Section 6.3, and the experiments evaluation and our main findings are presented in Section 6.5. Finally, Section 6.6 contains some conclusions and future work.

\subsection{Overview}

In opportunistic networking, there is an on-going evolution on how data-bundles (i.e., messages) are interchanged among users. This trend is basically due to the rapid increase in either the number of mobile users and of mobile accesses to services and information. Mobility introduces links with intermittent connectivity due to various reasons, such as path changes, overhead for path maintenance, propagation effects, etc. Applications, in parallel, especially cloud-based or webbased ones, use HTTP as a "transport" layer protocol, relegating TCP to a simple short-duration contact provider. This approach is increasing the flexibility and the resilience to the mobility of many new services and paves the way to simplify the introduction of the "opportunistic dissemination" of messages. This chapter focuses on this type of data distribution approach and studies the combined use of fixed and mobile nodes to establish an urban opportunistic network aimed at the distribution of general interest data.

In this chapter, we evaluate the combined use of fixed and mobile nodes to establish an urban opportunistic network aimed to push common interest messages in this scenario. Fixed nodes were used either as relays and as message producers/consumers. We consider two very large realistic GPS vehicular traces belonging to cities with different street structures, namely Rome [18] and San Francisco [124]. Through simulations, we compare the message delivery probability, latency and the network overhead. These experiments were carried out using two different simulators - The ONE and OMNeT++.

To evaluate the work presented here, an opportunistic networking node model has been built that includes the relevant protocol layers from the application layer to the dissemination layer, opting for a simple link layer.

The results show that mobility and density are the critical factors, driving the performance of opportunistic networks. These results are very important for the development of future services, as they contradict the trivial and widely spread assumption that using fixed nodes always "helps". Moreover, we believe that this chapter contributes significantly to provide a better understanding of the dynamics of mobile opportunistic networks.

The authors of $[46,16]$ exploited the social interaction as an indicator to improve the message ratio delivery and to optimize the energy resources. The previous studies emphasized on data traffic and cooperation in pedestrian contexts. Their results cannot be easily extended to vehicular contexts where the mobility scenario changes drastically due to the higher relative speed of nodes. But authors like [31] showed the importance of vehicles participating actively in the 
distribution of messages, since they can provide: a relative abundance of memory, constant energy availability, computational resources and the availability of geographical information through GPS devices.

Related with this area, in [177], the authors characterize a total of three vehicular traces in China, two from Shanghai (bus and taxis), and one from Shenzhen. In $[35,137,152]$ the authors offer a wide application of vehicular networks detailing where and how to employ certain communication approaches. They clearly establish the differences between MANETs, VANETs and VDTNs (Vehicular Delay Tolerant Network), concluding that the high mobility of vehicles leads to short contact durations limiting the amount of data transferred. They explore new routing protocols and new interaction mechanisms to improve the collaboration and data transmission in VANETs and VDTNs.

The authors of [99] used another trace set of 4,000 taxis, validating the collected data, and creating their own mobility model called Shanghai Urban Vehicular Network (SUVnet). They evaluated message diffusion performance by comparing through simulation two different DTN routing protocols: the non-geographic pure Epidemic routing and their new proposed geographic DTN routing, the DistanceAware Epidemic Routing (DAER). They also compared, as mobility models, the Random Way Point model, a SUMO-generated model, and their own SUVnet. The results obtained assigned the best performance when using the conventional Random Way Point mobility model. This conclusion is critical since it forces to use more realistic models and to use microscopic data to better evaluate new proposals.

About the optimization of resources in mobile devices, there are other studies in $[40,117]$, where the authors consider the message organization of message in the buffer, and show how to improve the message forwarding between mobile devices. It is important to highlight that the aforementioned works, use in their experiments short-range radio interfaces, such as Bluetooth and WiFi.

Differently from the above cited works, in this chapter we evaluate the combined use of fixed and mobile nodes to establish an urban opportunistic network aimed at the distribution of general interest data. We consider two very large realistic vehicular traces belonging to cities with different street organizations, namely Rome and San Francisco. Through simulations, we compare the message delivery probability and the network overhead. These experiments were carried out using two different simulators - The ONE and OMNeT++.

\subsection{Message Diffusion Proposal}

The first goal of this section is to describe the different opportunistic communication strategies that were considered to exchange the messages in our scenarios detailing its parameters. Then, we evaluate the combined use of fixed and mobile 
nodes to establish an urban opportunistic network aimed at the distribution of general interest data.

\subsubsection{Fixed nodes assisting mobile nodes}

The first approach is based on using the vehicles (in this scenario the mobile nodes are concretely the taxi cabs) as "Producers", "Consumers" and "Relays" of messages $(\mathbf{P} / \mathbf{C} / \mathbf{R})$, and the fixed nodes only act as relays $(\mathbf{R})$ of the messages. In other words, the fixed nodes support the mobile nodes.

The main motivation behind this scenario is the widely spread assumption in opportunistic networks research (for example in [45]) that fixed nodes at some strategic places, such as tourism offices, police or train stations, would significantly improve the delivery delay and delivery ratio by relaying messages also when none of the mobile nodes are around. In other words, if you arrive late at the train station, your messages will be waiting for you there, even if none of your friends is around. As we will show later in the chapter, this assumption does not hold in general.

The explanation example of the Figure 6.1a is function of the time and position of the nodes when a message is created and this is disseminated in the wireless network. In a time $t_{1}$ the vehicle $V_{3}$ passes near to the fixed node $F n_{2}$ placed on the geographical position $P_{2}$ (the nodes contact), in this time $F n_{2}$ does not have messages in its buffer from another cab, and it does not transmit anything to $V_{3}$. In a second time instance $t_{2}, V_{1}$ in the position $P_{1}$ produces/generates a message $M_{1}$ with destination $V_{3}:\left(M_{1}\left(V_{1}, V_{3}\right)\right)$, this vehicle contacts with the fixed node $F n_{1}$ and the message is stored, and it is available in the $F n_{1}$ buffer; while this is happening in a $t_{3}$, the rest of cabs exchange messages in the opportunistic network. In the time $t_{4}$ the cab $V_{2}$ contacts with $F n_{1}$ and the message $M_{1}$ is forwarded to $V_{2}$, in the instant $t_{5}$ when $V_{2}$ is in the geographical position of $F n_{2}$, the message $M_{1}$ is forwarded to the relayer fixed node $F n_{2} . M_{1}$ is available in $F n_{2}$, and in a time $t_{n}$ when $V_{3}$ in in the range of transmission $P_{2}$ to $F n_{2}(50 \mathrm{~m})$, the message is forwarded from this fixed node to $V_{3}$ (destination node).

This is the dynamic of the message diffusion using fixed nodes (additional to mobiles nodes). The advantage of this approach is that the messages are available in the fixed nodes buffer, and the vehicles can get a copy of the messages of another mobile nodes (cabs) that were on this geographical position.

\subsubsection{Mobile nodes assisting fixed nodes}

In this second approach, the fixed nodes are the "Producers", "Consumers" and "Relays" of messages $(\mathbf{P} / \mathbf{C} / \mathbf{R})$, and the vehicles act as mules or relay nodes $(\mathbf{R})$, bringing messages from one fixed node (source) to another fixed node, until the final fixed node destination is reached (destination). 


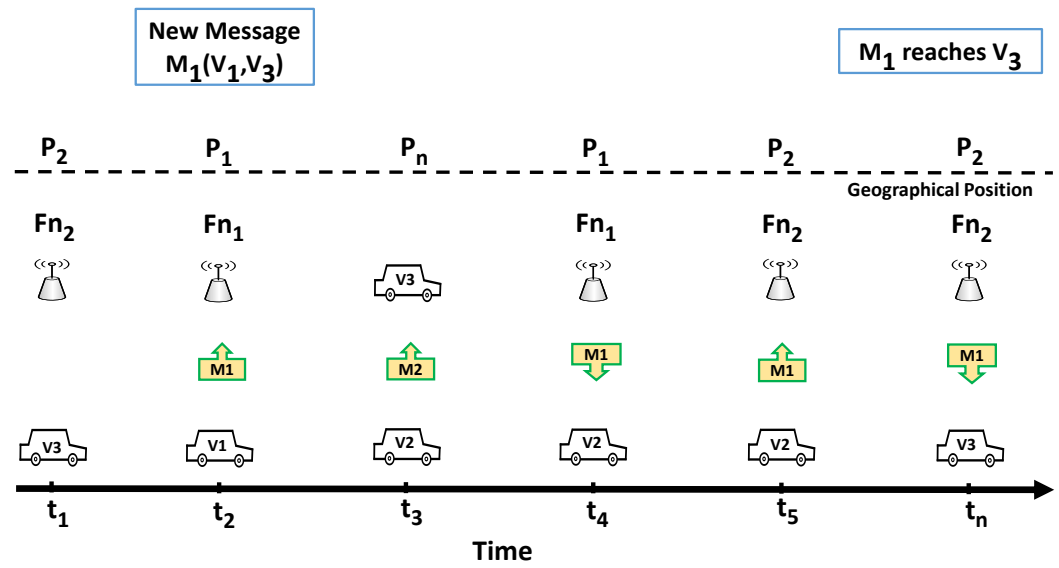

(a) Cabs are $\mathrm{P} / \mathrm{C} / \mathrm{R}$, and fixed nodes as $\mathrm{R}$.

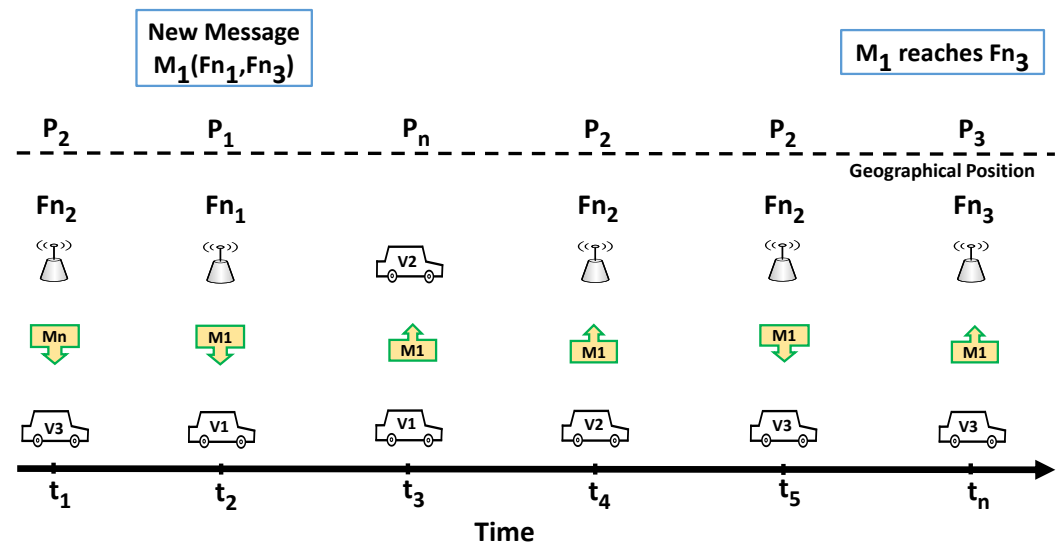

(b) Fixed nodes are $\mathrm{P} / \mathrm{C} / \mathrm{R}$, and cabs as $\mathrm{R}$.

Figure 6.1: Different roles of the cabs and fixed nodes. 


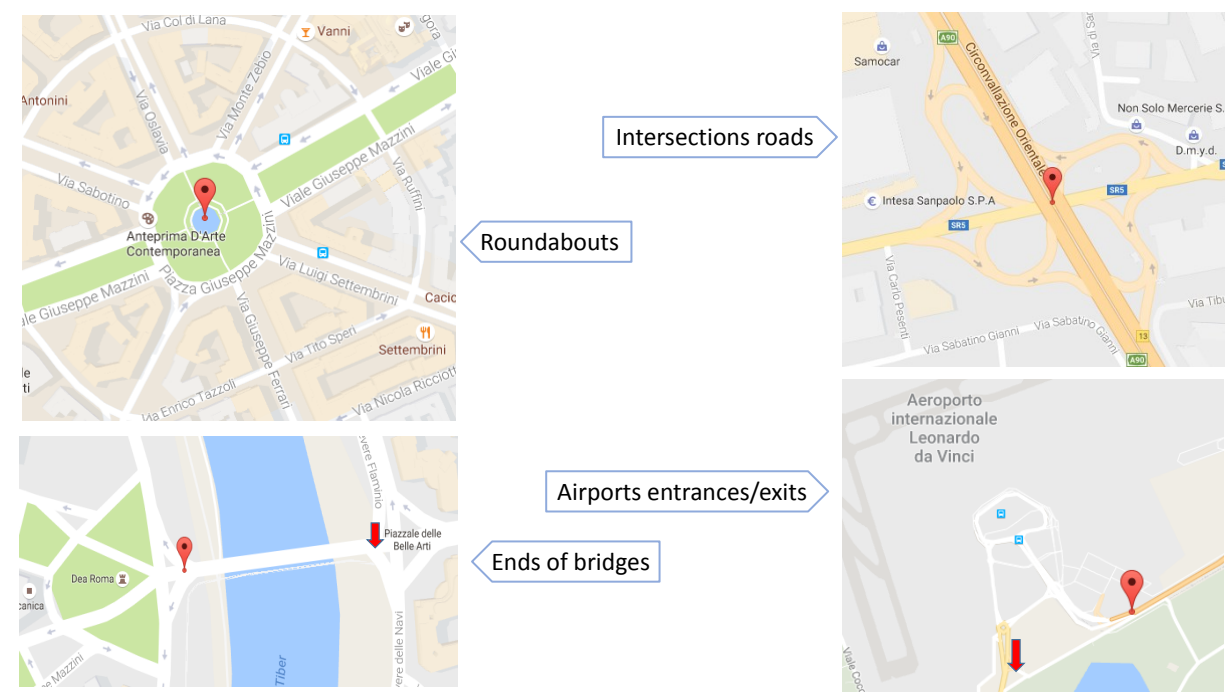

Figure 6.2: Criteria to place the fixed nodes.

In our example, the Figure $6.1 \mathrm{~b}$ illustrates the spreading process of the message $M_{1}$ created in a geographical position $P_{1}$ by the fixed node $F n_{1}$ with a destination fixed node $F n_{3},\left(M_{1}\left(F n_{1}, F n_{3}\right)\right)$. The message is forwarded by mobiles nodes (cabs) between the rest of the nodes until reach to his destination, in this specific case $\mathrm{Fn}_{3}$ in the $P_{3}$ geographical position.

The motivation here is derived from the world of wireless sensor networks and their use of "data mules" for sensed data delivery. However, the main difference is that our data mules are not controlled and travel on their own without being aware that they are serving the purposes of our application. However, many scenarios are possible where the mules can be very helpful: for example to send messages from traffic lights to the police station in a Smart City context.

\subsubsection{Node placement strategy}

A good question is where to place the fixed nodes. This problem was faced by taking as reference a grid, in order to avoid concentration of fixed nodes, and by looking for specific strategic geographical locations in the target scenario. We considered areas with high user concentration probability, like touristic places (squares, main buildings, museums, etc.), and areas with high traffic probability, such as roundabouts, road intersections, ends of bridges, among others; Figure 6.2 illustrates the criteria. Considering the transmission range, in our case $50 \mathrm{~m}$, when the selected places were too large we put more than one fixed node in order to cover the whole area. 


\subsection{Description of Experiments}

The main goal of this chapter is to experimentally validate the assumption that fixed nodes can potentially improve the performance of mobile opportunistic networks. Moreover, we also investigate the opposite scenario: can mobile nodes assist non-connected fixed nodes? In the first case, we speak about mobile nodes serving as sources and destinations, while in the second case the fixed nodes are used either as sources or destinations.

For all the experiments we assume that data messages are created at all nodes according to a Poisson traffic generator with an average value of one message every two hours. The size of each message is $1 k B$ and has a single random destination (a node ID). We suppose that, when two nodes meet, they interchange the data messages they do not have in their respective buffers. Once a message gets delivered to its final destination, it is not propagated further and it is marked as received. These events are used to calculate the delivery ratio and the delivery latency.

The parameters we consider are the following (see also Table 6.1):

- Time-to-Live (TTL): maximum live time for every individual message. Once this period expires, the message is deleted and considered not delivered.

- Number of nodes in the network: The maximum available nodes are 536 for the San Francisco trace (SFO), and 316 for the Rome trace. We also run experiments with fewer nodes to evaluate the evolution of the performance.

- Number of fixed nodes: we explore the performance of the system by varying the number of fixed nodes and their location.

The Epidemic message diffusion is carried out when mobile nodes contact and have the opportunity to exchange their missing messages. The upper part of

Table 6.1: Simulation parameters varied to evaluate message diffusion.

\begin{tabular}{|c|c|}
\hline Parameter & Values \\
\hline \hline Buffer Size & $1 G B$ \\
\hline Routing & Epidemic \\
\hline Mobile nodes & 316 (Rome), 536 (San Francisco) \\
\hline Fixed nodes & $0,27,55,108,216,337$ \\
\hline Interval & $1 \mathrm{msg}$ per node $/ 7200$ secs \\
\hline Time to Live & 12,48 hours \\
\hline Bandwidth & $2 M b / \mathrm{s}$ (WiFi-Direct) \\
\hline Tx-Range & $50 m$ (WiFi-Direct) \\
\hline
\end{tabular}


1)
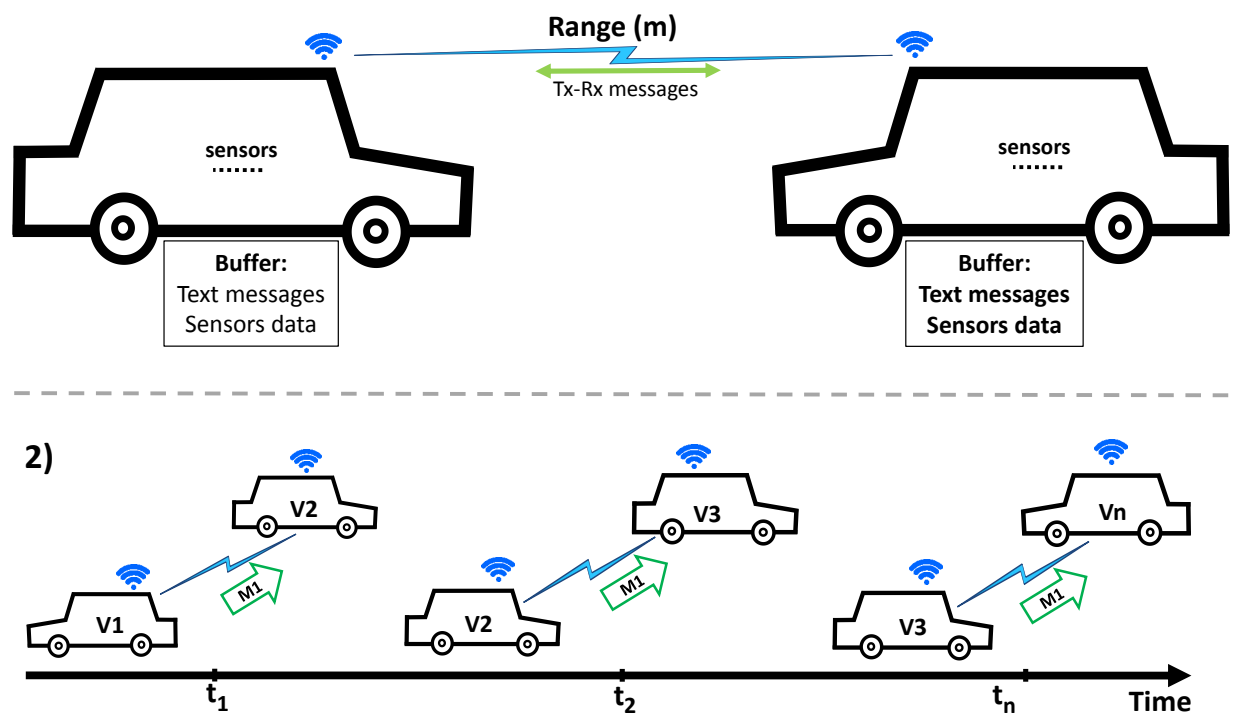

Figure 6.3: WiFi interfaces embedded in vehicles.

Figure 6.3, illustrates the approach to exchange data among vehicles using WiFiDirect technology, where the data could be generated by sensors embedded in these mobile devices. On the bottom part of this figure an example of the epidemic message dissemination scheme is shown, where the message $M_{1}$ is transmitted from a vehicle $V_{1}$ to $V_{2}$, after some time $V_{2}$ sends the message to $V_{3}$ when both vehicles are in communication range, and the process continues until the message arrives to its destination.

\subsection{Performance Evaluation}

In this section we explore the dynamics of opportunistic networks using The ONE simulator with two large real vehicular movement traces, San Francisco (SFO) and Rome, and the OMNeT++ simulator with one of these traces (SFO), and generating a network load based on a Poisson traffic generator with message size of $1 \mathrm{kB}$. We decided to explore the behavior of the network with two different simulators, because the first results showed very low impact and we wanted to exclude the possibility that the results were tool-biased. 
In the next paragraphs, we first present the properties of the GPS traces themselves. Next, we describe our main results by presenting the experimental data and their evaluation.

\subsubsection{The GPS traces}

We use two sets of vehicular traces in our experiments. The first trace (Rome), was already described in section 5.4. The second GPS trace (SFO) has about 11 million points and comes from 536 cabs in San Francisco during 24 days [124]. The covered area is, however, smaller than the Rome trace with only $40 \mathrm{~km} \times 40 \mathrm{~km}$. Thus, the average density of this trace is approximately 10 times more than the Rome trace. We again converted the GPS records to Cartesian coordinates, centered at San Francisco's business center.

Figure 5.2a and 6.4a show how the vehicular traces are distributed around the metropolitan areas in Rome and San Francisco cities respectively. Figures 5.2b and $6.4 \mathrm{~b}$ are zoomed views of the previous ones, showing how the main urban area of the cities are almost fully covered by the traces.

The parameters we use for our simulations (both OMNeT ++ and ONE simulators) are summarized in Table 6.1 and correspond to typical scenarios for opportunistic networks.

\subsubsection{Fixed nodes do not help}

The first question we asked is whether the trivial assumption that fixed nodes potentially support mobile nodes in the dissemination of data is true or not. Our results show that this assumption is not true. Figure 6.6 shows the experimental results, which lead us to this conclusion. The scenario is as follows: all mobile nodes of the traces serve as source/destinations/relays, while the fixed nodes "support" the mobile ones with relaying messages. The more fixed nodes we add, the larger the overhead (c), but neither the latency decreases (b), nor the delivery ratio (a) increases significantly. At the same time, we see some slight impact on the Rome traces, which are less dense. What we also see is our next observation: the denser the network, the better the performance.

\subsubsection{The denser the network, the better}

This observation is not surprising by itself, but the level of performance increase was unexpected. The Rome scenario has a mean density of 0.0316 nodes per square kilometer, while the SFO scenario has a density of 0.355 nodes per square kilometer. Looking at Figure 6.5, we see that higher nodes densities leads to a dramatic increase in the number of contacts, from around 150-200 contacts per hour for the Rome scenario to over 12,000 for SFO. This is a very important 


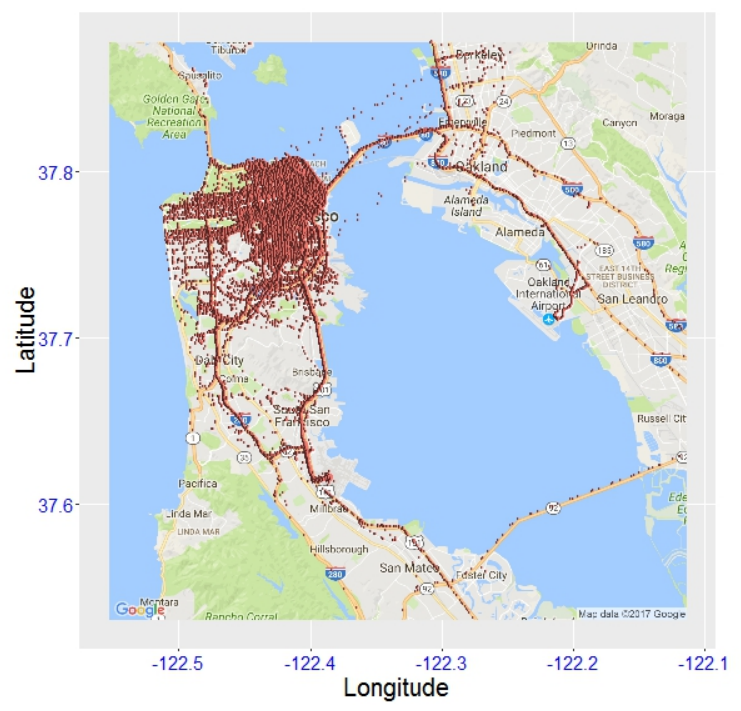

(a) Complete trace area.

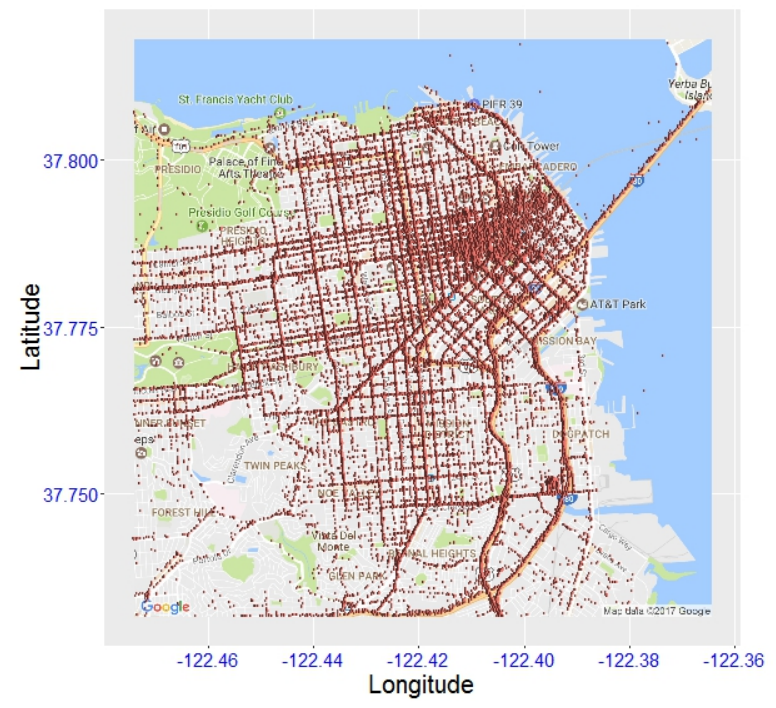

(b) Zoomed-in area of the city center.

Figure 6.4: San Francisco trace sample of $6 \mathrm{~h}$ of GPS traces. Total length of trace is 24 days, 536 taxis in an area of $1,600 \mathrm{~km}^{2}\left(0.335\right.$ nodes per $\left.\mathrm{km}^{2}\right)$ 


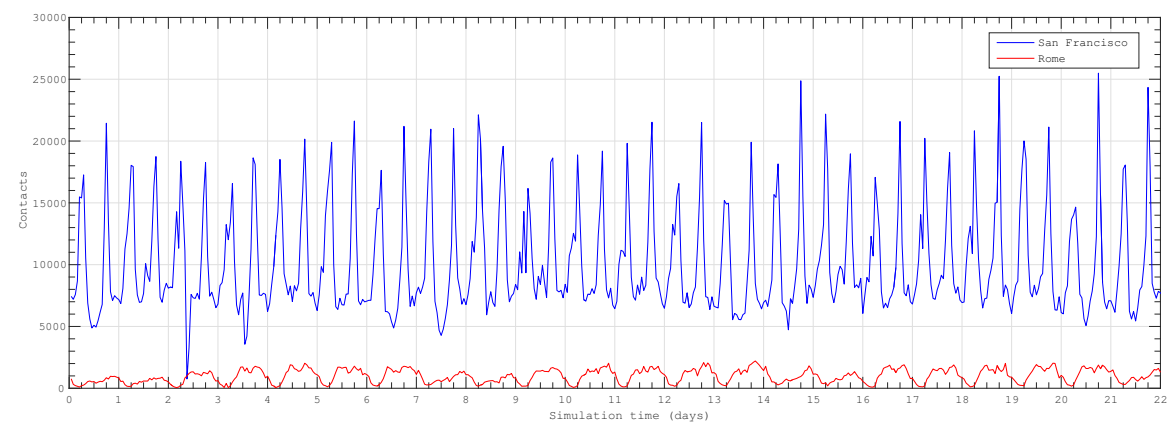

Figure 6.5: Contacts per hour generated by the simulation for each taxi trace.

observation, as the density is still very low compared to the fixed network nodes, but already delivers quite a good performance, as we will also see later.

\subsubsection{Mobility helps}

Now, let us revert the scenario as explained in Section 6.3 and explore what happens with a fixed network, supported by more and more mobile nodes. The results are again as expected, but not at that level of significance. The experimental results are shown in Figure 6.7. While the fixed network is completely disconnected by itself, as can be seen by the first point in the graph with no mobile nodes at all, even a small number of mobile nodes improve dramatically the delivery rate and the latency. For example, the delivery ratio increases from $8 \%$ for the Rome traces with a TTL of 48 hours and only 10 mobile nodes to $50 \%$ for the same experiment but with 100 mobile nodes. This is an increase by a factor of approx. 6 . The overhead increases from 30 to 100, a factor of only 3.3 - half of the increase in the delivery rate. Thus, we can conclude that adding mobile nodes increases significantly the performance of the network at a relatively low cost.

\subsubsection{Mobile nodes are more important than fixed nodes}

In order to further explore and understand the dynamics of mobile against fixed nodes, we performed the following experiment with an opportunistic network that uses the Randomized Rumor Spreading (RRS) [83] as its data propagation strategy. Concretely, we simulated a number of network scenarios where the number of nodes in the network was kept constant (100) while changing the ratio of fixed nodes to mobile nodes from $100 \%$ to $0 \%$ in each of the scenarios. The data was generated always by $30 \%$ of the nodes to keep the number of generated data constant for all the scenarios. The mobile nodes use the SFO traces to obtain their movement pattern while the fixed nodes are positioned in a grid across the mobility 


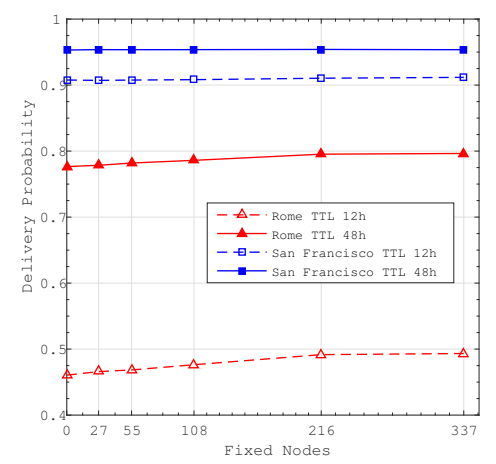

(a) Delivery Probability.

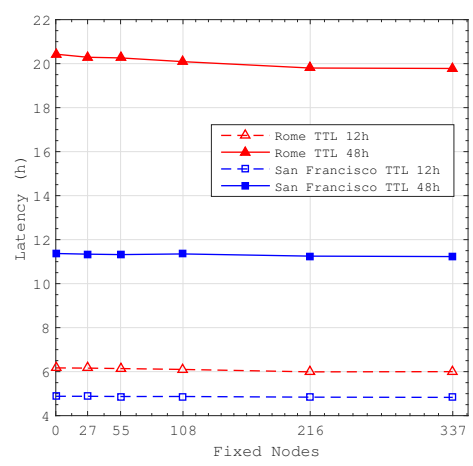

(b) Latency time (hours).

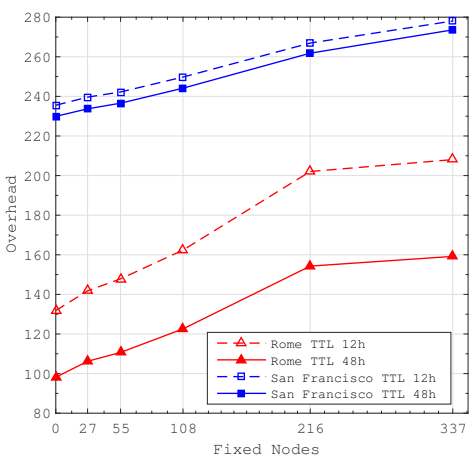

(c) Overhead (forwarded messages divided by delivered messages).

Figure 6.6: Average delivery success ratio, latency and overhead. Mobile nodes act as sources and destinations, and fixed nodes as relays. 


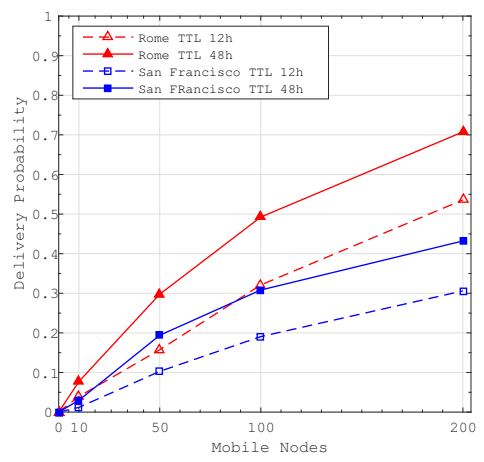

(a) Delivery Probability.

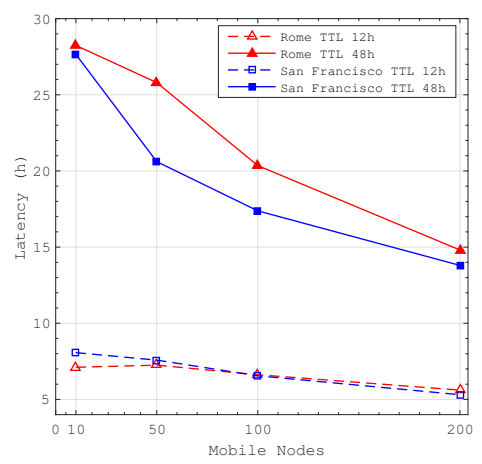

(b) Latency time (hours).

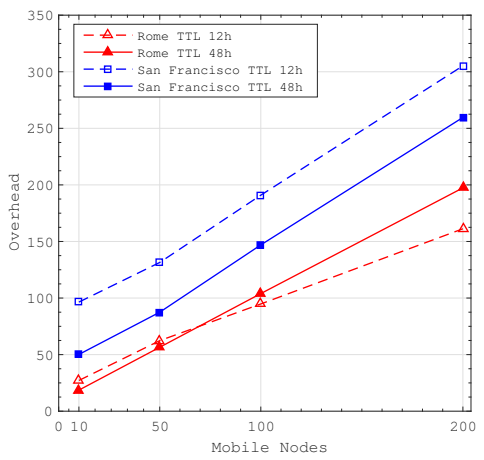

(c) Overhead (forwarded messages divided by delivered messages).

Figure 6.7: Average delivery success ratio, latency and overhead. Mobile nodes act as relays, and fixed nodes are the sources and destinations of messages. 


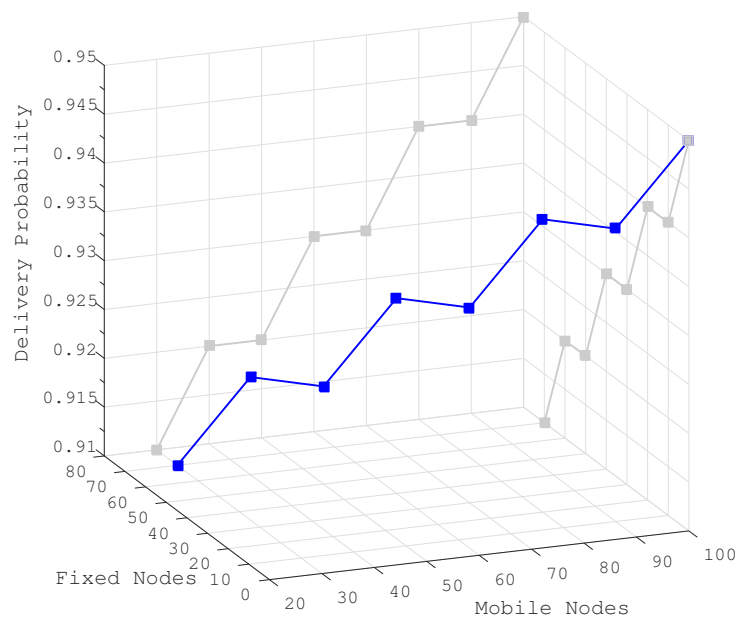

(a) Delivery Probability.

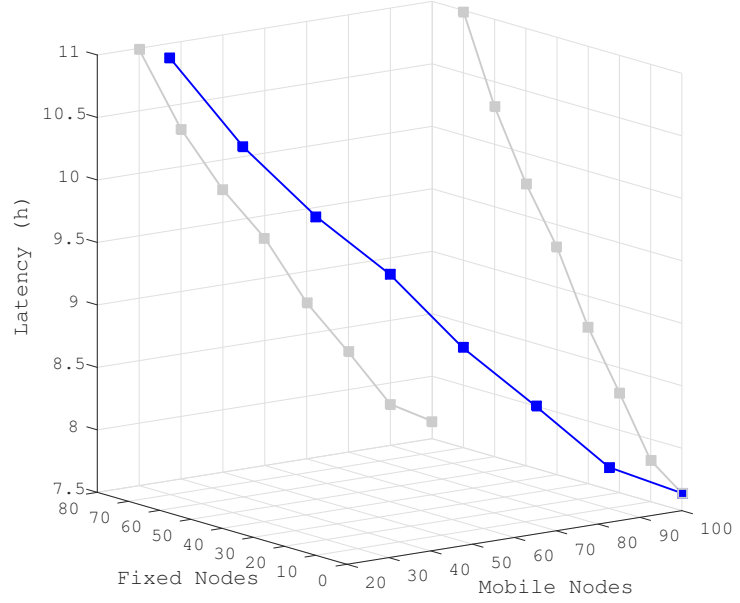

(b) Latency Time (hours).

Figure 6.8: A network with 100 nodes with different percentages of mobile against fixed nodes. There are always exactly 30 nodes producing data in the network and random destinations. The scenario is San Francisco. 
area. Figure 6.8 shows the obtained results. The results show that the substitution of mobile nodes in place of fixed nodes improves the amount of data delivered to the intended recipients and further reduces the delivery times of the data. Therefore, it can be said that introducing mobile nodes, even in a small quantity can bring better network performance than fixed nodes as the fixed nodes may end up being islands of caches without the possibility of passing on cached data.

\subsubsection{Delay is always significant}

The community has been discussing for a long time now what are the advantages and disadvantages of opportunistic networks. In the last paragraphs, we have shown how much potential mobility offers by easily connecting very sparse networks. However, we confirmed also one large disadvantage: the delay is significant in all cases. This is an intrinsic property of opportunistic networks and probably will remain the main challenge for future research. However, it has to be mentioned here also, that the delay is probably due to the destination-oriented way of diffusing messages. If you consider an alert application, where everybody in a certain radius around has to be notified quickly, we expect the delay to be really low. We will focus on this in our future work.

\subsubsection{OMNeT ++ versus The $\mathrm{ONE}$}

As mentioned in the beginning of this section, we did the experiments described here both in the OMNeT ++ and ONE simulators. Even if the used models (mobility traces, epidemic routing, transmission radius of 50 meters, etc.) were the same, the implementations are very different due to different programming languages and different simulation kernels. It is good to report that the results can be considered identical, with differences below $0.1 \%$. However, the simulation in $\mathrm{OMNeT}++$ runs faster than ONE: for a simulation of 50 nodes (among the smallest we had here) the ONE needs more than 11 hours, while OMNeT++ finishes in 2.5 hours on the same hardware configurations. This is probably due to the fact that OMNeT++'s kernel is written in $\mathrm{C}++$ and ONE in Java.

\subsection{Summary}

In this chapter, we evaluated the combined use of fixed and mobile nodes to establish an urban opportunistic network aimed at the distribution of general interest data. We used simulations based on two real-world movement traces from taxi cabs of Rome and San Francisco, with a workload characterizing a large text messages applications. We used WiFi-Direct as the data transmission technology.

The idea of adding fixed nodes came originally from the observation that the mobile users of a specific opportunistic service might not be sufficient to build a network dense enough and that fixed nodes at strategic places could complement 
the mobile ones. We determined that fixed nodes only increase the overhead while mobile nodes boost the performance significantly. We showed that, as expected, mobility is very crucial for the performance of opportunistic networks. It is not the hindering and challenging property of these networks, but their main enabler. We found that nodes density does not have to be very high, but that a higher density significantly improves the performance. We observed that the number of contacts is critical, a point that will require anyway further investigation.

Combining these findings, we can summarize that, to increase the performance in opportunistic networks by increasing the node density, should be obtained by adding mobile nodes - not fixed ones. Mobile nodes are able to boost the performance, while fixed nodes only increase the overhead. Thus, when needed, adding mobile nodes like for example on buses, trains or taxis, is the best strategy to efficiently establish an urban opportunistic network. 


\section{Chapter 7}

\section{Impact of the Data Transfer Time}

\subsection{Introduction}

The Epidemic protocol is an effective way to achieve information diffusion in opportunistic networks. Its performance depends on two key factors: the device mobility pattern and the message transmission time. The mobility pattern determines the contact time and duration. If contact durations are shorter than the required transmission times, some messages will not get delivered, and the whole diffusion scheme will be seriously hampered.

In this chapter we evaluate the impact of message transmission times in Epidemic diffusion processes. We demonstrate how, when certain conditions hold, changing the mobility routine to stop moving until completing the data delivery process can improve the diffusion performance. We implemented this mobility model, called Full-Stop, in The ONE simulator, and we show that, for large message sizes, the diffusion performance is increased.

The outline of the chapter is as follows: an overview of related efforts addressing opportunistic networks and message diffusion is presented in section 7.2. The description of our mobility proposal, experiments and evaluation details are presented in Sections 7.3 and 7.5, respectively. Finally, in section 7.6 a summary of this chapter is presented.

\subsection{Overview}

In opportunistic ad-hoc networks, the intermittence and short duration of contacts between pairs of mobile devices are prevalent. This model is applicable to envi- 
ronments where there is no defined topology or wireless infrastructure for data transmission. Currently, this is a subject addressed by many researchers, who are designing and implementing their protocols and applications to address the associated challenges.

In order to evaluate the performance of the dissemination protocols we used The ONE simulator, defining a geographically-confined scenario, where a social event is taking place (e.g., a festival, a fair, or a demonstration) with moderate assistance of people, who can share thematic information through direct terminalto-terminal messaging.

Along with the opportunistic networking model also emerged novel routing protocols capable of storing and carrying information between mobile devices. When adopted in real situations, the network behavior is epidemic; authors in $[116,129]$ specifically focus on evaluating the performance of this information dissemination approach.

There are many research works about spreading messages on a social opportunistic network. In [16] the authors examine an utility-based cooperative data dissemination system where the utility of data is defined based on the social relationships between users. They studied and validated the performance of this system through an analytical model, allowing to characterize the data diffusion process. Furthermore, they analyzed the behavior of the system with respect to key parameters such as the definition of the data utility function, the initial data allocation on nodes, the number of users in the system, and the data popularity.

In this context, the authors of [173] used theoretical analysis applied to social networks to classify and study some diffusion schemes based on the "homophily" (social networks phenomenon) by combining node relationships and their interests in the data. They noticed that, to improve diffusion performance, a node should first diffuse the most similar data according to their common interests when meeting a friend, and it should first diffuse the most dissimilar data according to their common interests when meeting a stranger. In [168] a detailed analytical model is proposed to analyze the epidemic information dissemination in mobile social networks. This model is based on rules concerning user behavior, especially when their interests change according to the information type, and this can have a considerable impact on the dissemination process. Then, the model is developed through ordinary differential equations, taking account that information can only be delivered from one node to another when they meet and are socially connected with each other. Based on long simulations, they have demonstrated the accuracy of this model.

Differently from the aforementioned proposals, other authors had instead a contact-time perspective, and relied on different mathematical models. The authors of [165] present an analytical study describing the performance of the Epidemic protocol, arguing that intermittently-connected mobile networks can be modeled as edge-Markovian dynamic graphs. A new model is proposed for Epidemic propagation on such graphs, and they calculate a closed-form expression 
that links the best achievable delivery ratio to common opportunistic network parameters such as message size, maximum tolerated delay, and link lifetime. This research shows that, given a certain maximum delay and node mobility, the bundle size has a major impact on the delivery ratio.

In the same context of analytical research, the authors of [60] propose a model based on Delay Differential Equations to evaluate the diffusion of messages in groups taking into account the transmission time of the messages. This model was validated through simulation.

The authors of [4] introduce a mathematical approach for message diffusion in opportunistic networks using the Epidemic protocol. This approach is based on well known models for the spreading of human epidemical diseases, e.g. SIR [86]. One of the main conclusions of this analysis (mathematical model and its respective simulation) is that SIR models are quite accurate for the average behavior of Epidemical DTN, even in discrete scenarios with a relatively low number of nodes. Furthermore, a Montecarlo method is proposed to address the issue of standard deviation, since deviation values were higher than average values.

Channel contention is one of the factors considered to evaluate the Epidemic protocol. In [76] the authors analyzed the contention issue, identifying three factors that affect dissemination in opportunistic networks, which are: (i) the finite bandwidth of the link, which limits the number of packets that two nodes can exchange; (ii) the scheduling of transmissions between nearby nodes, which is needed to avoid excessive interference; and (iii) the interference from transmissions outside the scheduling area. Therefore, analytical expressions are formulated to examine the diffusion, and through simulation confirmed the bound of their theoretical model. The authors suggest that these factors must be considered in the analysis of communications protocols.

There are other proposals such as $[110,103,148,43]$, which evaluate the message dissemination behavior of the Epidemic protocol by focusing on the mobility patterns of the nodes. In these works, the authors explain the relationship between factors such as speed, mobility model, density of nodes, and places. In addition, some of these authors propose their own mobility model to improve the diffusion in opportunistic networks. There are also some works [158, 130, 63] where the authors explain how to improve the dissemination process of epidemic protocols in order to save battery energy.

The aforementioned studies provide an overview of recent research works providing analysis and performance improvements for the Epidemic protocol. These articles show the importance of this protocol concerning opportunistic networking, and the trend towards models and applications that exploit the particularities of delay-tolerant wireless networks. However, these works do not analyze the performance of the Epidemic protocol taking into consideration the relationship between the contact duration time, and the message size. We focus on this aspect and we evaluate the impact on data distribution of this factor. 


\section{3 $\quad$ Message Diffusion Approaches}

In this section we detail the different schemes for the diffusion of the messages and the modification performed in The ONE simulator in order to evaluate them.

\subsubsection{Message Diffusion Scheme}

The basis of contact-based messaging is to establish a short-range communication directly between mobile devices, and to store the messages in these devices in order to achieve their full dissemination; no messages are sent or stored in servers. Message spreading is based on epidemic diffusion, a concept similar to the spreading of infectious diseases. Basically, when an infected node (i.e., a node that has a message) contacts another node, it infects it (by transmitting the message). Epidemic routing obtains the minimum delivery delay at the expense of an increased usage of local buffers, and an increased number of transmissions.

The dissemination scenario works as follows: various mobile devices are supposed to have a messaging application that notifies and shows to the user any received messages for the subscribed groups. The application is cooperative i.e., it must store the received messages and perform the diffusion of such messages to other nearby nodes. Each node has a limited buffer to store the messages received from other nodes. When two nodes establish a pairwise connection, they exchange any messages stored in their buffers and check whether some of the new messages are suitable for notification to the user.

A critical factor that determines whether a contact is long enough for a complete transfer of messages is the application behavior when nodes contact each other. Two approaches can be assumed:

- The devices are forced to stop when they need to exchange information. In this case, the owners of the mobile devices control this exchange by stopping and waiting until the message transmission is completed. This is a commonly used scheme in several existing short-range mobile protocols such as Apple iOS Airdrop [156], and Google Android Copresence [51].

- The devices do not stop. In this case, the completion of message transmission will depend on the contact duration. The result is that, if the contact duration is lower than the message transmission time, the transmission will fail. Thus, we must assume that some contacts will not complete their message transmission.

The second approach, clearly the most flexible from the user, experience point of view, raises an interesting question: how much time do we assume users/nodes contacts last based on the considered mobility model? This will clearly depend on the users, mean speed and the communication range, as detailed in the evaluation section. 


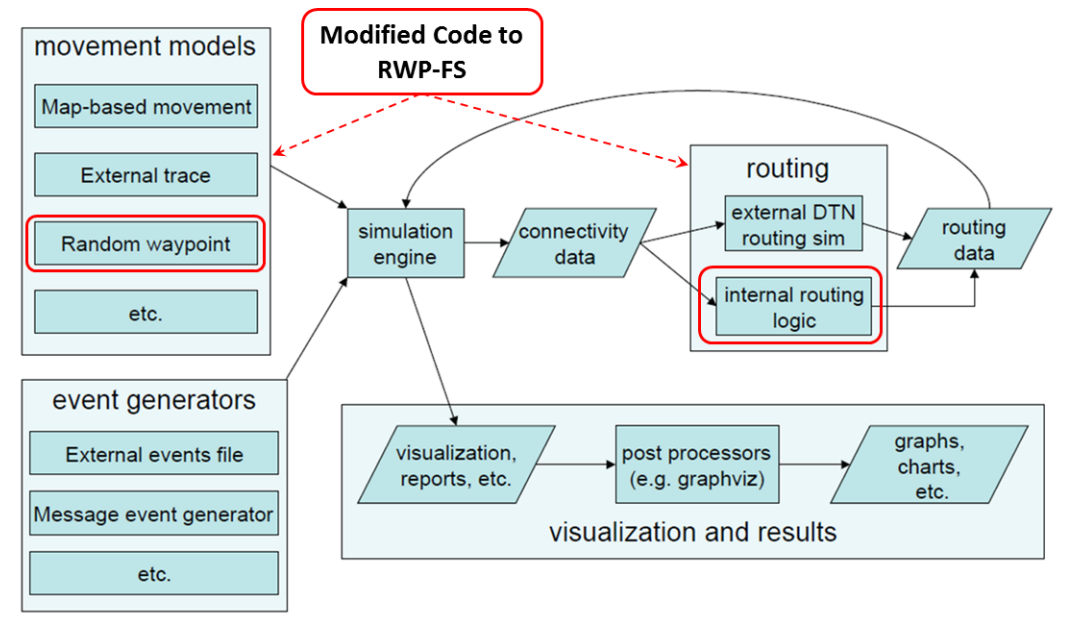

Figure 7.1: The ONE simulator structure, where the red boxes indicate the modified modules. (The figure is based on the original from [85]).

In this chapter is tested the spreading properties of the epidemic protocol by considering the Random Waypoint (RWP) movement model, and by introducing a novel variant that we called RWP-FS (Random-Way Point - Full Stop), as detailed below.

\subsection{Description of Experiments}

The experiments were performed using The ONE simulator with the modifications described in section 7.4.1. We considered the following mobility models:

1. RWP: In the Random Way-Point movement model, the parameter "waiting time" (wt) defines the maximum waiting time that a node has to be motionless after reaching the next position.

2. RWP-FS: where nodes are forced to stop until the transmission is completed.

3. Static: All nodes are steady. Although this is not actually a real mobility model, it is used in some experiments to compare results with other mobility models. 
Table 7.1: Simulation fixed parameters.

\begin{tabular}{|c|c|}
\hline Parameter & Value \\
\hline \hline Protocol & Epidemic \\
\hline Nodes & 500 \\
\hline Nodes speed $(\mathrm{m} / \mathrm{s})$ & $0.25-0.75$ \\
\hline Interface & Bluetooth \\
\hline Tx radio range $(m)$ & 7.5 \\
\hline TX Bandwidth $(M b p s)$ & 2.1 \\
\hline Buffer & unlimited \\
\hline TTL $(\min )$ & 1,500 \\
\hline
\end{tabular}

\subsubsection{The ONE simulator modification}

The RWP [13] mobility model has been extended to force both nodes in a transmission to stop until the transmission ends. This extension is called RWP-FS (Random Way Point - Full Stop) model. RWP-FS is a more accurate model of the human behaviour than regular RWP when people share messages with mobile devices in a given space. Figure 7.1 shows which modules in ONE were modified to implement this extension. The main code changes are in the ActiveRouter, DTNHost, and Connection Java classes, implementing Algorithm 2. Devices are forced to stop every time they have a message to transmit (the speed of both nodes is set to $0 \mathrm{~m} / \mathrm{s}$ ). Any stopped device will start a new movement (according to mobility parameters with a speed "n_speed" randomly chosen between 0.25 to $0.75 \mathrm{~m} / \mathrm{s}$ ) only when the transmission is actually over.

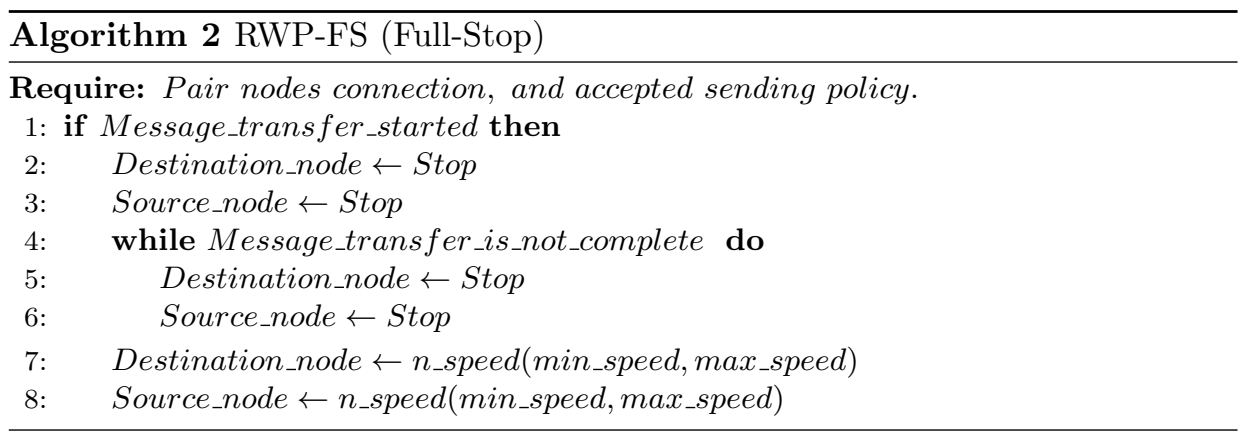

All the experiments performed simulate the diffusion of one message in an area where a fixed set of nodes move. More specifically, we consider a square area with variable length side $l$, where 500 nodes can move freely, with a mean 
Table 7.2: Simulation variable parameters.

\begin{tabular}{|c|c|}
\hline Parameter & Value \\
\hline \hline \multirow{3}{*}{ Density (Area side) } & 0.05 nodes $/ \mathrm{m}^{2}(l=100 \mathrm{~m})$ \\
& 0.1 nodes $/ \mathrm{m}^{2}(l=70 \mathrm{~m})$ \\
& 0.2 nodes $/ \mathrm{m}^{2}(l=50 \mathrm{~m})$ \\
\hline & $1 k B$ \\
Message size & $1 M B$ \\
\hline & $10 M B$ \\
Movement model & RWP wt 0 $(s)$ \\
& RWP wt 0-120 (s) \\
& RWPS \\
& Static \\
\hline
\end{tabular}

speed of $0.5 \mathrm{~m} / \mathrm{s}$. The communication range $(r)$ is $7.5 \mathrm{~m}$ with a mean bandwidth of $B w=2.1 \mathrm{Mb} / \mathrm{s}$, and setup time $T_{s}=0.1 \mathrm{~s}$. These values were selected based on a Bluetooth 2.0, Class 2 specifications. Class 2 radios have a range of up to $10 \mathrm{~m}$, but we assume a certain interference and thereby reduce its coverage to $7.5 \mathrm{~m}$. Table 7.1 shows the values of the common parameters to the different scenarios, and Figure 7.2 shows a snapshot of the ONE simulator running with 500 nodes.

The parameters for each experiment are shown in Table 7.2. Three node densities are used: $0.05,0.1$, and 0.2 nodes $/ \mathrm{m}^{2}$, obtained using different area sizes for the same number of nodes (500). Regarding the message transmission, three message sizes are considered: a short text message $(1 \mathrm{kB})$, a low-resolution picture or photo $(1 M B)$, and a short video o high resolution picture $(10 M B)$. Finally, four mobility models are considered, with different waiting times. In all cases (except the static case) the node speed is uniformly distributed between 0.25 and $0.75 \mathrm{~m} / \mathrm{s}$. We ran over 360 repetitions of the dissemination process, 40 for each parameter combination, and we obtained their mean values.

\subsection{Performance Evaluation}

The goal of this section is to evaluate the diffusion of a message in a given area, under several conditions such as mobility models, people density, and message size. Furthermore, we present the percentage of messages aborted and forwarded for the different mobility models used.

First, we analyze the dynamics of the diffusion. Figure 7.3 shows the dissemination of one message of medium size $(1 M B)$, using RWP and RWP-FS mobility models. The sub-figures show the distribution of the number of nodes that receive the message. The bars in the plots represent the number of messages transmitted, 


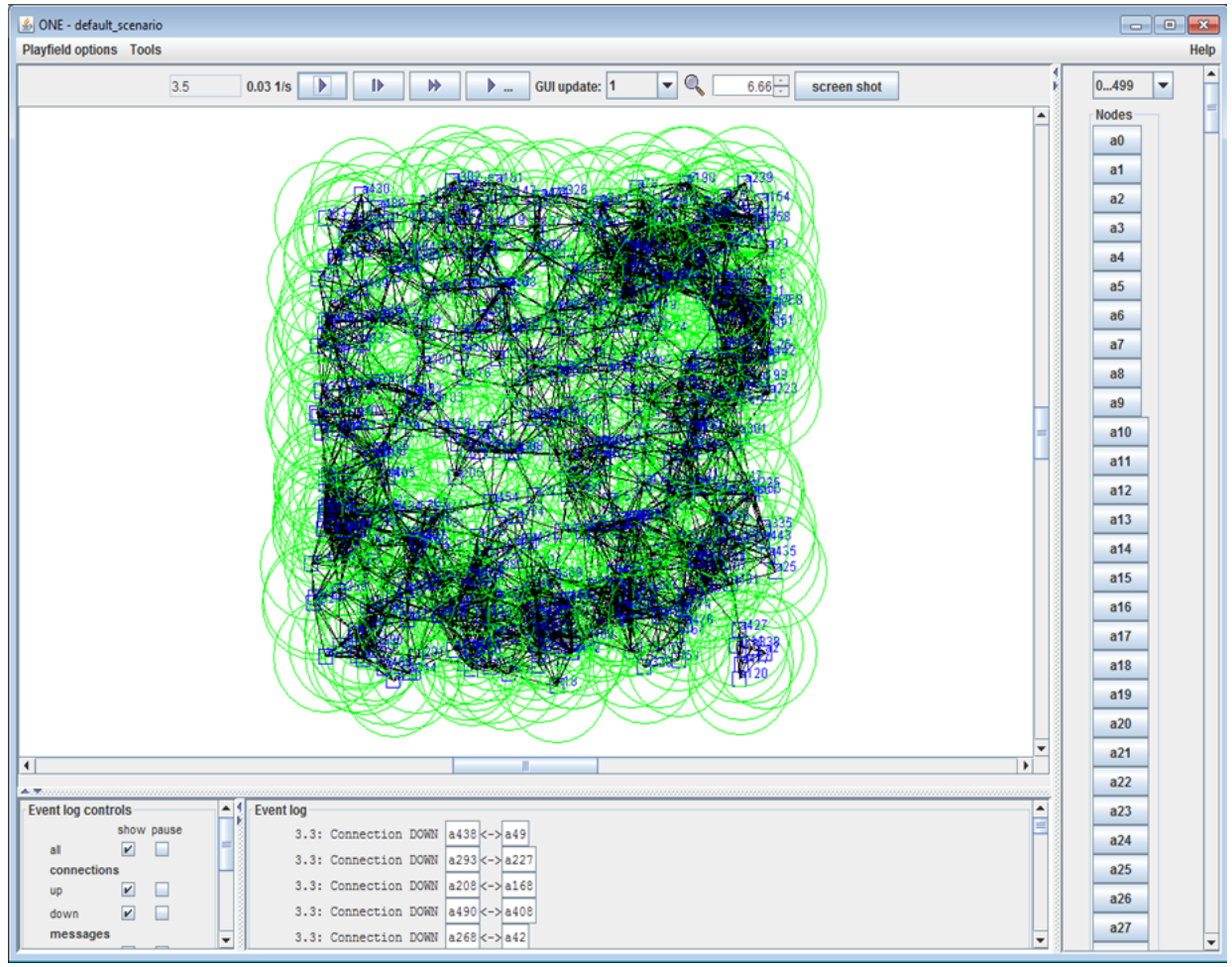

Figure 7.2: Representation of The ONE simulator running.

allowing us to evaluate the speed of the diffusion process. We can observe that the maximum diffusion speed takes place when approximately $50 \%$ of the nodes have been infected with the message. Beyond this threshold the diffusion speed becomes slower, a phenomenon that is mainly due to the reduced amount of nodes without the message.

The second row in Figure 7.4 provides a graphical representation of the message propagation process, where each point represents the time when a node has received the message. We can clearly see that dissemination patterns are quite different depending on the mobility models. For the RWP mobility model, the dissemination depends exclusively on the mobility of nodes and their contacts, and this is reflected with an apparent random distribution of the points. Nevertheless, for the Full-Stop model (RWP-FS), we can clearly see that points (reception of messages) are periodically concentrated in rows, doubling the number of receptions by a period that is proportional to the required transmission time of $4 \mathrm{~s}$. When the number of remaining nodes is low, this periodical propagation becomes scattered over time due to the reduced number of remaining nodes without the message. 


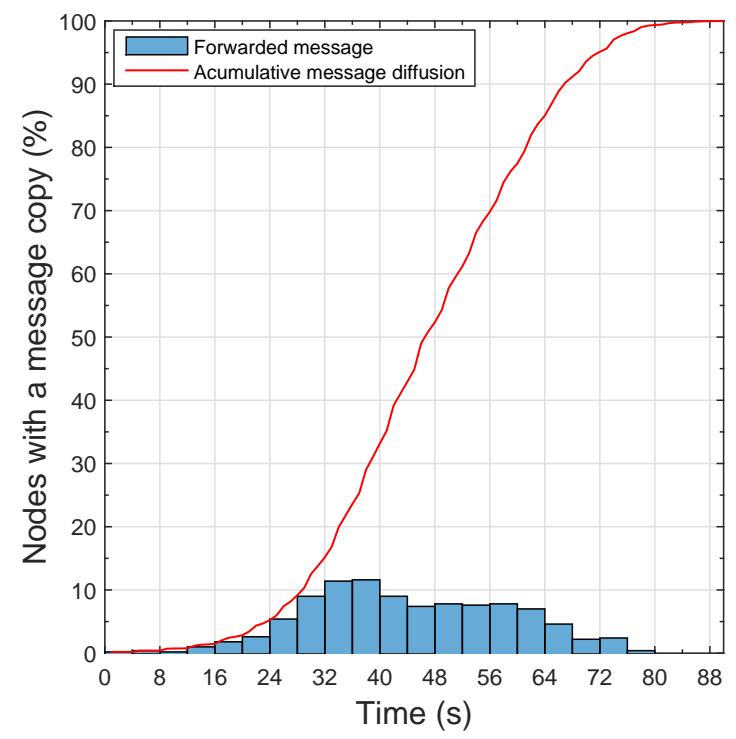

(a) Message $1 M B$, RWP wt 0-120s

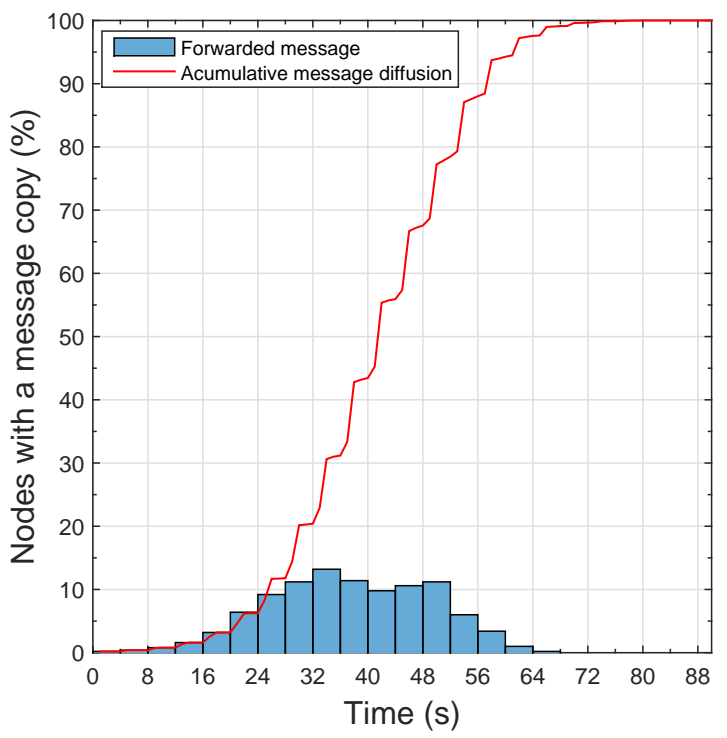

(b) Message $1 M B$, RWP-FS

Figure 7.3: Dynamics of the message diffusion, according to movement models. 


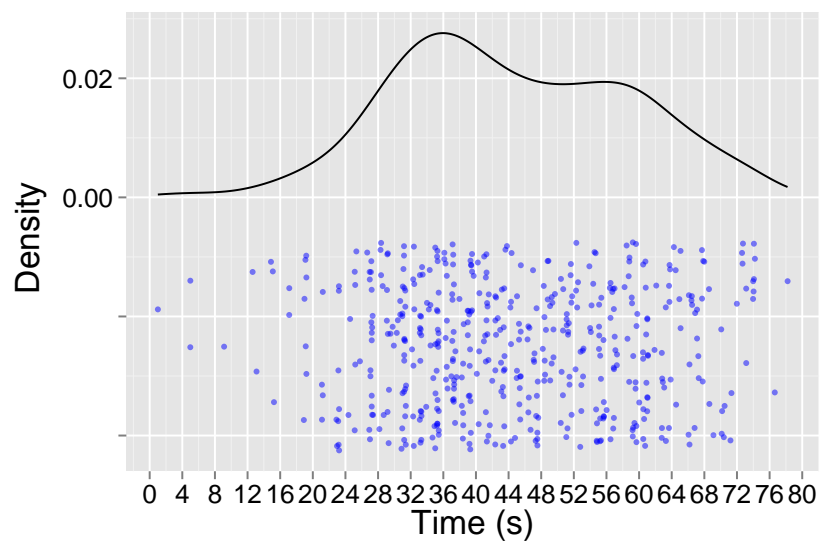

(a) Message $1 M B$, RWP wt $0-120 \mathrm{~s}$

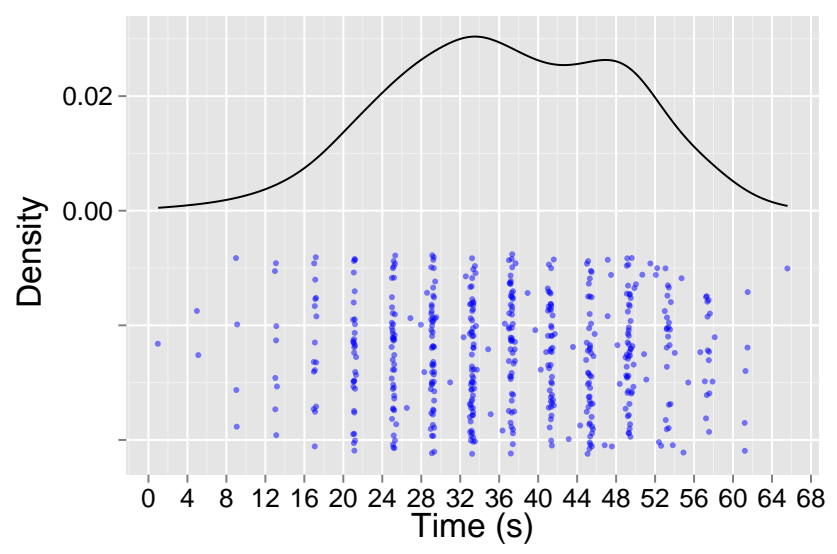

(b) Message $1 M B$, RWP-FS

Figure 7.4: Dynamics of the message diffusion, according to movement models. 


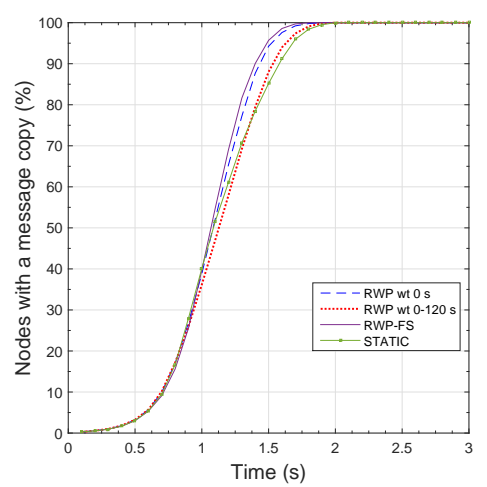

(a) Message $1 \mathrm{kB}$, Density 0.2 nodes $/ \mathrm{m}^{2}$

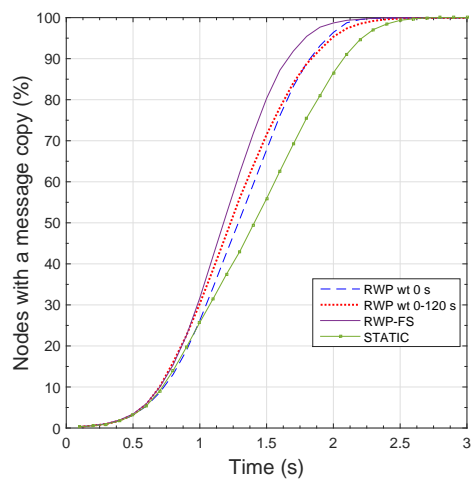

(b) Message $1 \mathrm{kB}$, Density 0.1 nodes $/ \mathrm{m}^{2}$

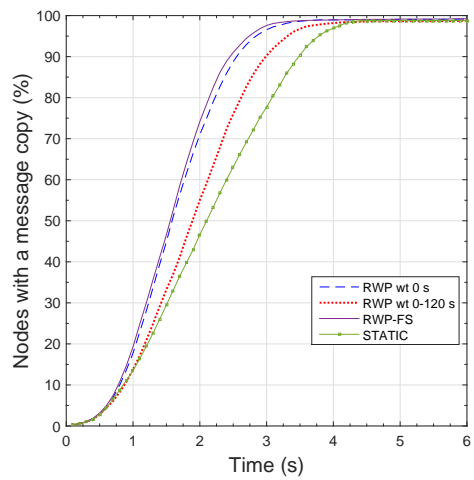

(c) Message $1 \mathrm{kB}$, Density 0.05 nodes $/ \mathrm{m}^{2}$

Figure 7.5: Message $1 k B$ diffusion according to size, density, and movement models. 


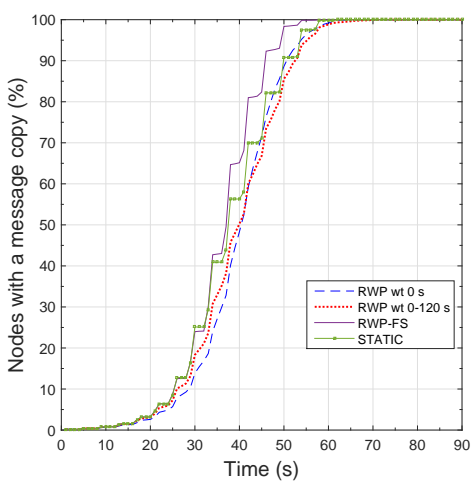

(a) Message $1 M B$, Density 0.2 nodes $/ \mathrm{m}^{2}$

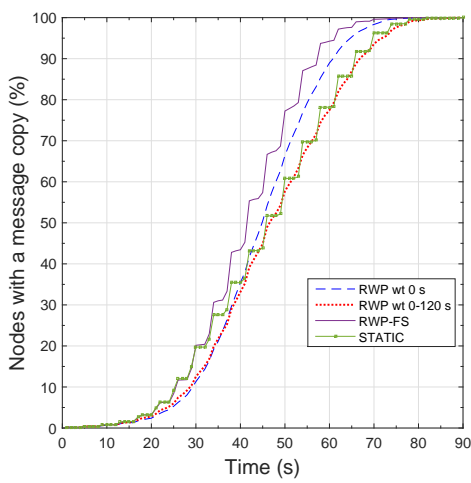

(b) Message $1 M B$, Density 0.1 nodes $/ m^{2}$

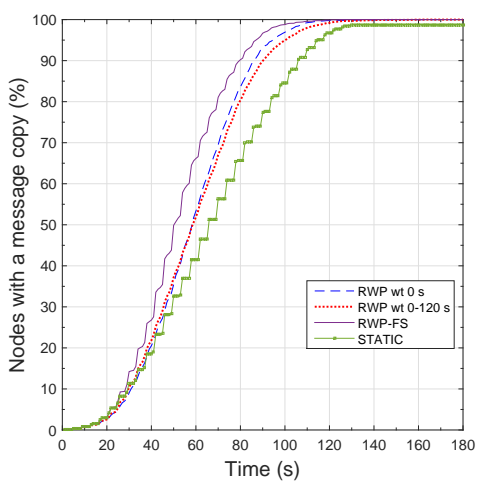

(c) Message $1 \mathrm{MB}$, Density 0.05 nodes $/ \mathrm{m}^{2}$

Figure 7.6: Message $1 M B$ diffusion according to size, density, and movement models. 


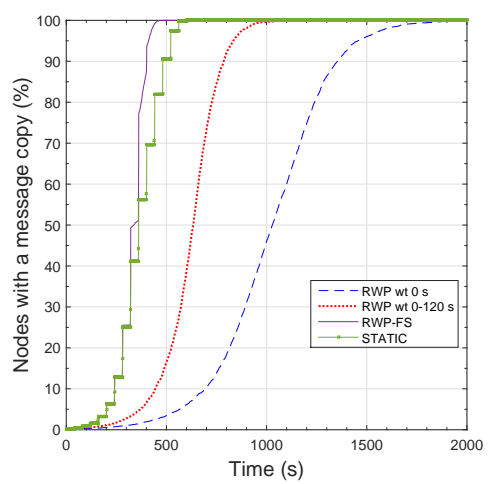

(a) Message $10 \mathrm{MB}$, Density 0.2 nodes $/ \mathrm{m}^{2}$

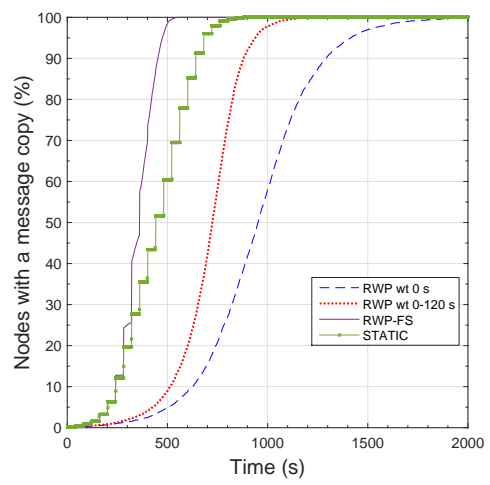

(b) Message $10 M B$, Density 0.1 nodes $/ m^{2}$

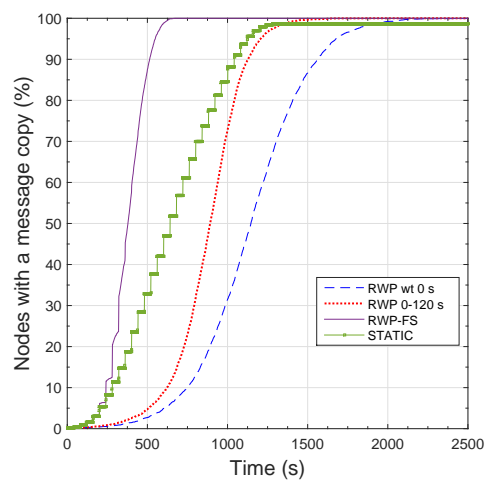

(c) Message $10 \mathrm{MB}$, Density 0.05 nodes $/ \mathrm{m}^{2}$

Figure 7.7: Message $10 M B$ diffusion according to size, density, and movement models. 


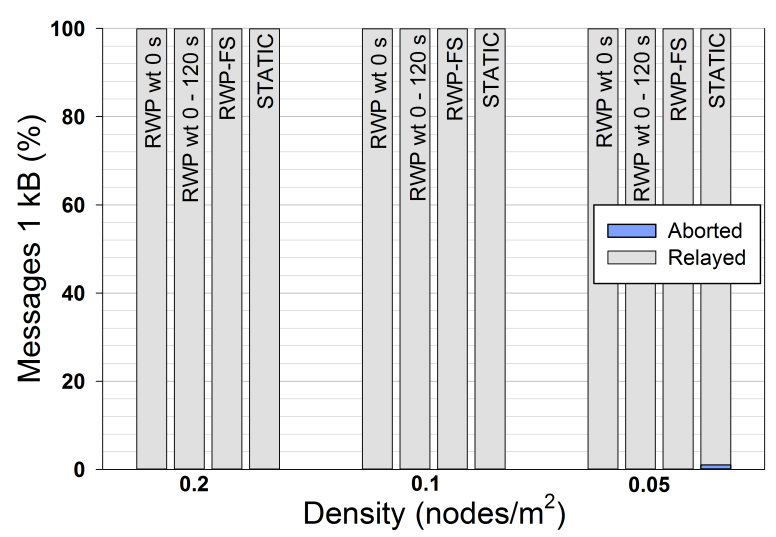

(a) Message $1 \mathrm{kB}$

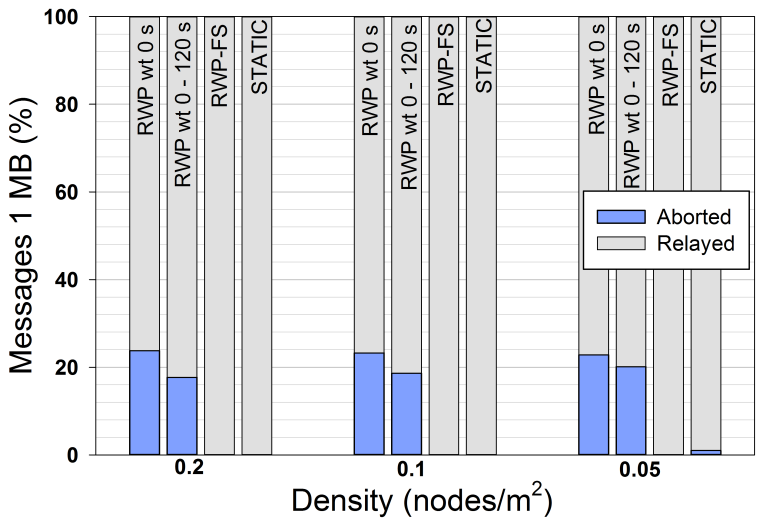

(b) Message $1 \mathrm{MB}$

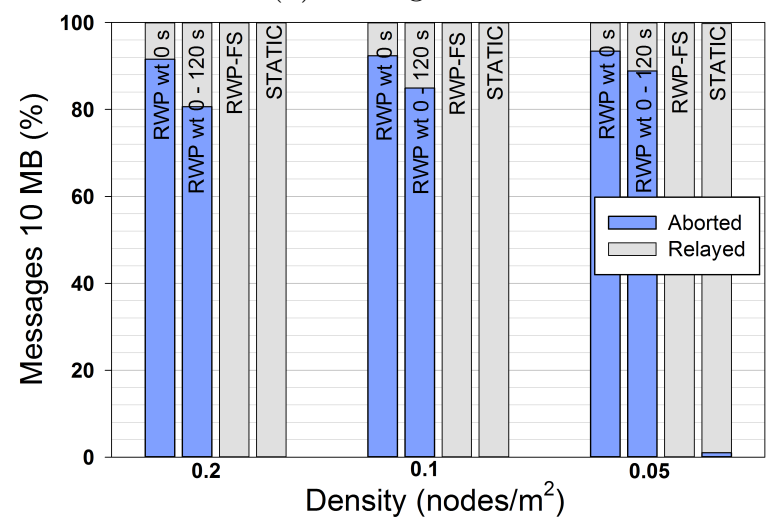

(c) Message $10 \mathrm{MB}$

Figure 7.8: Percentage of aborted and relayed messages. 
Figures 7.5, 7.6, and 7.7, show how the number of nodes receiving the message evolves over time under different mobility models. The different plots belong to the three different message sizes $(1 k B, 1 M B, 10 M B)$, and these are arranged according to the different node densities $\left(0.2,0.1\right.$, and nodes $\left./ \mathrm{m}^{2}\right)$.

As expected, the message is forwarded faster when node densities are higher. For example, dissemination is $200 \%$ faster with a density of 0.1 nodes $/ m^{2}$ than for a density of 0.05 nodes $/ \mathrm{m}^{2}$. Nevertheless, if we compare the spreading speed in the smallest area, the message spreading is about $20 \%$ faster than in a mediumsized area. This is because node density directly affects the transmission process of messages.

We now focus on the mobility model and the message size. In general, the best results are obtained using RWP-FS, that is, forcing a stop between nodes to complete the message transmission. Depending on the message size, we can see that, for short messages ( $1 k B$ - in Figure 7.5$)$ the difference between mobility models is reduced. This is an expected result, as the required contact duration to transmit a message is very low. Nevertheless, for larger messages $(10 \mathrm{MB}$, in Figure 7.7), the difference between the mobility models becomes quite evident. The worst performance is obtained with the RWP wt $0 s$. We can conclude that the mobility pattern provided by this mobility model does not favor a faster propagation of the message, since the contact duration is not enough to completely transfer messages. For RWP wt 0-120s the results are a slightly improved, since node stops result in longer contact durations.

As a reference, the plots also include the results when the nodes are static. When node density is high (0.2) results are very similar to the RWP model. Nevertheless, for lower densities, diffusion efficiency is reduced, resulting in larger diffusion times.

The previous experiments show that forcing a stop until the message is transmitted improves message diffusion. When mandatory stops are not enforced, transmissions will depend on the duration of contacts. If a transmission cannot be completed for the duration of a given contact, it is considered an aborted transmission. Thus, to conclude the evaluation, Figure 7.8 shows the amount of aborted and relayed messages depending on message size, mobility model, and node density. In Figure 7.8a, there are no aborted messages for short message sizes $(1 \mathrm{kB})$, thereby confirming that there is enough time in every contact to transmit this message. However, for larger messages, the number of aborted transmissions is increased in the RWP movements models, in particular for $10 M B$ sizes, where the number of aborted transmissions grows beyond 90\%. In the RWP-FS mobility model, the number of aborted transmissions is zero, because delivery is guaranteed by the full stop.

Finally, for static nodes (no mobility), some of the nodes can be isolated and cannot contact with other nodes. This is evident in low density areas, as shown in the graph for density 0.05 nodes $/ \mathrm{m}^{2}$, where about $2 \%$ of nodes cannot receive the message. 


\subsection{Summary}

In the first part of this chapter, it was analyzed the performance of message dissemination using epidemic diffusion. This performance depends mainly on the device mobility pattern and the message transmission time. We prove that, when contact duration time is shorter than the required transmission time, the performance is seriously reduced. Thus, we propose to force devices (properly speaking, their users) to stop until the message is completely transmitted. This implies a new mobility model, called Full-Stop, which was implemented in The ONE simulator.

The experimental evaluation shows that changing the mobility of nodes until the message is delivered improves the message diffusion process. When mandatory stops are not enforced, the transmission success will depend on the duration of the contacts. Therefore, if the message size is big (such as images or short videos), the number of aborted transmissions increases significantly, thereby reducing the overall performance. 


\section{Chapter 8}

\section{Improving the Message Diffusion Performance}

\subsection{Introduction}

In the previous chapters, opportunistic wireless networks have been evaluated considering determinant factors such as the type of mobile users (pedestrian and vehicular) considering the impact of the data transfer time, mobility patterns, message diffusion based on buffer management, and power consumption. And, specifically in a vehicular context, the opportunistic networks have been evaluated using alternative approaches in order to increase the message dissemination installing additional fixed nodes, and new technologies for data transmission such as LoRa.

From the previous evaluations, we conclude that the performance of mobile opportunistic networks strongly depends on contact duration. If the contact lasts less than the required transmission times, some messages will not get delivered, and the whole diffusion scheme will be seriously affected.

Therefore, in order to increase the diffusion efficiency we evaluated in the previous chapter the benefits of forcing to stop the nodes until the message is completely transmitted. In this chapter we refine and improve this idea. In first instance, the feasibility of this approach is evaluated under the name of Forced-Stop, evaluating its performance using pedestrian real traces. And, in second instance, and once analyzed the possibilities of this approach (Forced-Stop), it is proposed the Friendly-Sharing solution, which is based on Forced-Stop, but implementing control mechanisms and optimizations of resources in order to improve the message 
dissemination. Friendly-Sharing is evaluated using the same set of real traces. Furthermore in this section is explained the performance of the Android application GRChat, developed to exchange information between mobile users.

This chapter is organized in two big sections: in the first section the ForcedStop method is analyzed, and on the second section the Friendly-Sharing approach. Each section contains the respective related work, the message diffusion approach explanation, the evaluation by simulation process, and the presentation of the results.

\subsection{Related Efforts}

The proposed Friendly-Sharing approach is related to force collaboration between mobile users, so in this section we introduce some of the related efforts in this area.

The network performance of opportunistic networks is associated to the social relationships between users; based on this criterion, in $[28,46,16]$, the authors evaluate a utility-based cooperative data dissemination system, and propose a model of message dissemination considering key factors such as the allocation of nodes, the popularity and data utility. The idea of actively involving users with tasks of minimum impact on their regular activities is also employed by crowdbased participatory systems [23, 24].

The users, mobility is closely related to the routines of them, in [123], the authors introduce a new forwarding protocol, based on the PRoPHET algorithm, that incorporates the contact duration of encounters to compute the rating probabilities of all possible paths to a certain destination, augmenting the ratio of messages diffusion.

In order to improve the delivery ratio, some authors like $[122,142,167,151]$ have proposed new collaboration mechanisms between mobile users. Regarding collaboration or cooperative approaches, we can find research works that encourage collaboration through credit systems. More specifically, in [175, 21], the authors affirm that, when a cooperative system offers security and reliability, mobile users are more stimulated to cooperate, especially when the network includes several unknown users.

Finally, other studies analyze how the management of mobile device hardware influences the message delivery probability. In the case of buffer management, some authors $[40,42,117]$ evaluate the use of the buffer through priority rules in an attempt to deliver messages without performance loss in the information transmission process.

The aforementioned studies provide an outlook of the most recent research works focused on the analysis and performance improvements for the diffusion of messages by improving collaboration among mobile users. These articles also show the importance of cooperative behaviour in opportunistic networking, and 
the trend towards models and applications that exploit the particularities of delaytolerant wireless networks. However, these works do not analyze in an integrated way the performance of the message diffusion taking into account the duration of contact times, the buffer management schemes, and the relationship between the contact duration time and the message size.

\subsection{Forced-Stop: Feasibility of a new Message Diffusion Approach}

In this section we describe the operations and feasibility associated to contactbased message diffusion, and we propose a new scheme called Forced-Stop. Moreover, we detail the modifications introduced to The ONE simulator in order to evaluate this type of protocols. As stated previously, this experiment is a first approximation to proposed a new message dissemination method. Here we will just consider the action to stop the movement of the nodes when the nodes need to exchange information. On the next subsection is explained the performance of this approach.

\subsubsection{Contact-Based Message Diffusion Schemes}

The rationale of contact-based messaging is to establish short-range communications between mobile devices. A reference case study could be described as consisting of various mobile devices provided with a cooperative messaging application, that alerts and shows to the user any gotten messages in the groups that it has been joining. The application is capable to store and disseminate the received message from other nodes. When two close nodes establish a pairwise connection, they exchange any messages stored in their memory and check whether some of the new messages are suitable for notification to the user.

Message spreading is based on epidemic diffusion, a concept similar to the spreading of infectious diseases. Basically, when an infected node (i.e., a node that has a message) contacts another node, it infects it (by transmitting the message). Epidemic routing obtains the minimum delivery delay at the expense of increased buffer usage and number of transmissions. A critical factor that determines whether a contact is long enough for a complete transfer of messages is the nodes behaviour when a contact is established. Two variants are possible:

- The nodes continue their movement. In this case, the completion of message transmissions will depend on how long they remain within in communication range. If this contact duration is smaller than the message transmission time, the transmission will fail. 
- The nodes stop when they need to exchange information. In this case, the owner of each mobile device will control this exchange by stopping and waiting until the message transmission is completed.

Both approaches raise an interesting question: how much time do contacts last based on a particular mobility model? This clearly depends on average speed and communication range, as detailed in the evaluation section.

In this section is tested the spreading properties of the Epidemic protocol by considering both variants of people behaviour to share information. We propose the latter variant to improve the efficiency in data distribution, and we called it Forced-Stop (FS).

\subsubsection{ONE simulator modifications}

Figure 8.1 shows which modules in ONE were modified. First, in order to implement the Forced-Stop approach, we modified the ActiveRouter, DTNHost, and Connection Java classes. Devices are forced to stop every time they have a message to transmit by setting the speed of both nodes to $0 \mathrm{~m} / \mathrm{s}$. Any stopped device will resume its movement only when the transmission is actually finished.

Moreover, the original ONE message generator, in the MessageEventGenerator class, injects a new message using a random interval time. This random time is uniformly distributed from a range configured in the simulation parameters. In order to get a simulation closer to real human behaviour we modified this module

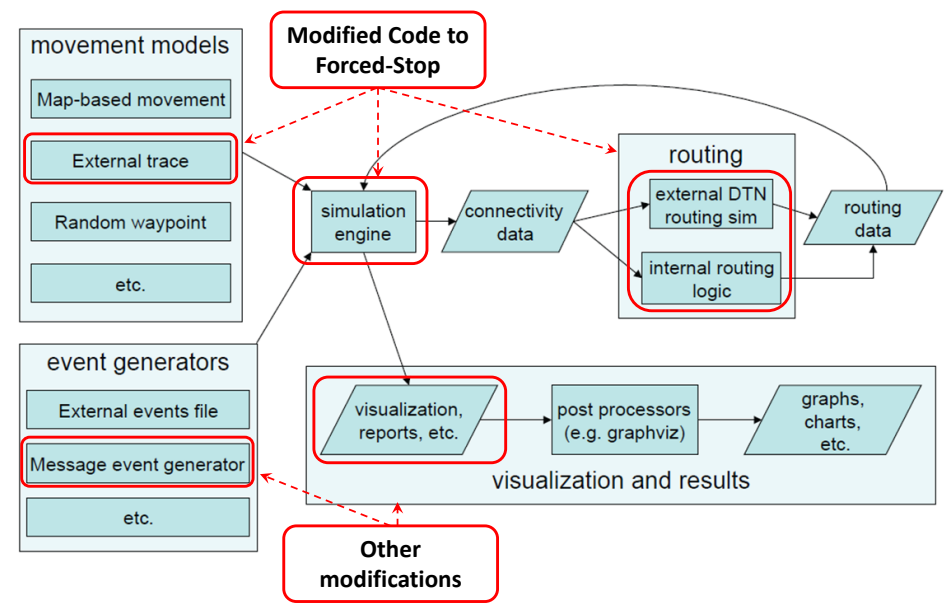

Figure 8.1: The ONE simulator structure, where the red boxes indicate the modified modules. (The figure is based on the original from [85]). 
to generate messages following a Poisson process, using an exponential random distribution.

Finally, although The ONE simulator produces a large variety of reports about the simulation process, there was no mechanism to obtain the buffer occupancy. We added a new report class that outputs the average and maximum buffer occupancy of all nodes for each step of the simulation. It also computes the maximum of the average buffer occupancy during the whole simulation.

\subsubsection{Description of Experiments}

The experiments were performed using The ONE simulator with the modifications described in section 8.3.2 using a real-life movement trace from an experiment at the NCCU University campus [154]. The NCCU Traces were collected using an Android app installed in the smartphones of students belonging to the National Chengchi University. A total of 115 students participated in the experiment. Their GPS data, application usage, WiFi access points, and Bluetooth devices in proximity were recorded over a period of two weeks. Time is specified with a resolution of one second, and the position information is rounded to meters. Figure 8.2 shows a snapshot of ONE running with the corresponding graphical map information. We considered both behaviour models presented previously: classical Epidemic and Forced-Stop (FS).

A key aspect on these experiments is the workload generation. Our generation pattern of messages is related with social networking applications. We considered a typical multimedia messaging application where each user generates messages of different sizes, and shorter messages are far more common than larger ones. This traffic generation is more detailed in section 4.4. Note that this workload is not the same used in [154], so the results presented here can differ from the ones presented in that paper.

Communication range $(r)$ was set to $7.5 \mathrm{~m}$ with bandwidth $B w=2.1 \mathrm{Mb} / \mathrm{s}$, and setup time $T_{s}=0.1 s$. These values were selected based on Bluetooth 2.0, Class 2 specifications. Although the maximum range is $10 \mathrm{~m}$, we assume a certain interference, and thereby we reduced the coverage value. Finally, the message Time To Live (TTL) was set to 12 hours or 24 hours, and buffer sizes varied from $50 M B$ to $1 G B$. The main simulation parameters are summarized in the Table 8.1 .

\subsection{Performance Evaluation of Forced-Stop}

The goal of this section is to evaluate the performance of message dissemination under the Epidemic Diffusion and Forced-Stop approaches.

We focus on the dynamics of the diffusion process. Figure 8.3 shows a sample diffusion graph, using a medium sized $(1 M B)$ message for dissemination. In this 
Table 8.1: The main simulation parameters.

\begin{tabular}{|c|c|}
\hline Parameter & Values \\
\hline \hline Buffer Size & $50 M B, 100 M B, 200 M B, 1 G B$ \\
\hline Message size & $1 k B, 1 M B, 10 M B$ \\
\hline Routing & Epidemic, Forced-Stop \\
\hline Time to Live & 12 hours, 24 hours \\
\hline
\end{tabular}

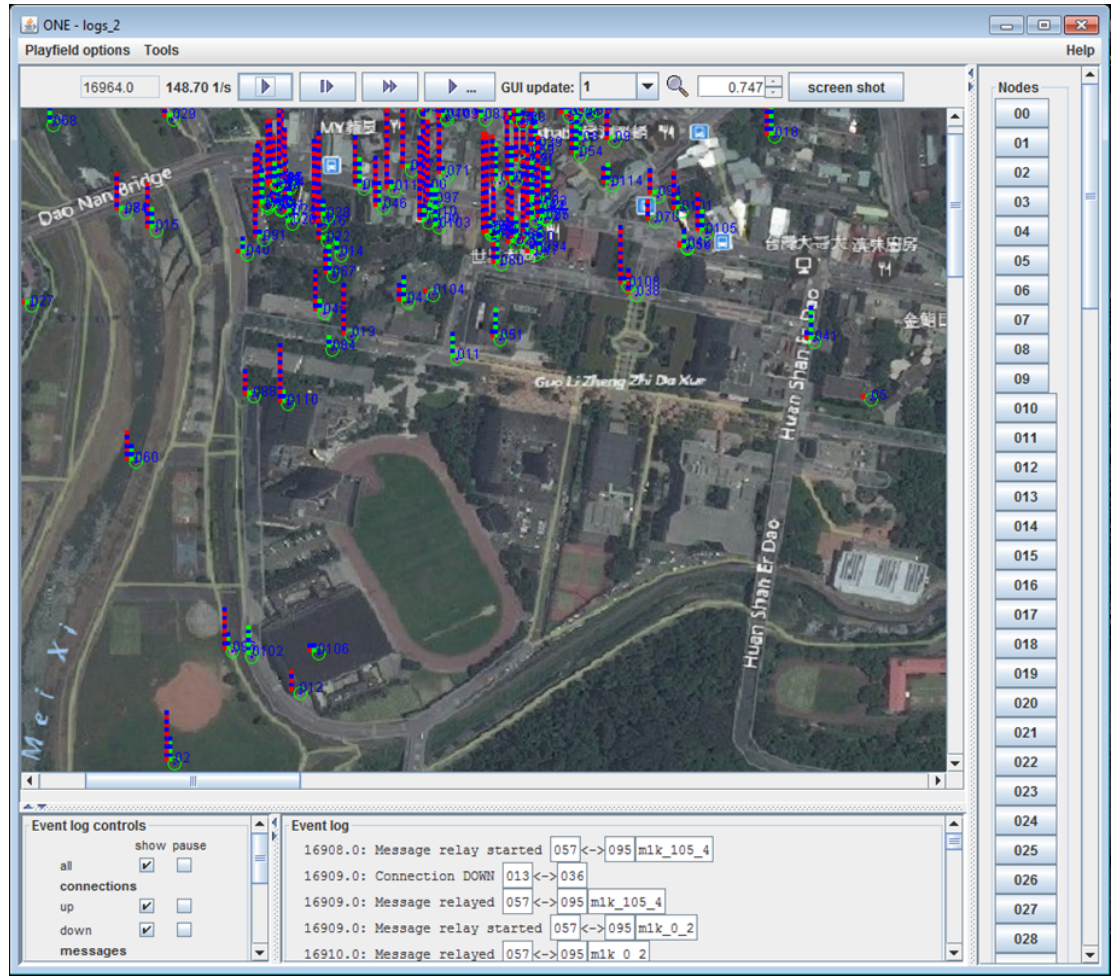

Figure 8.2: The ONE simulator running with the NCCU traces. 


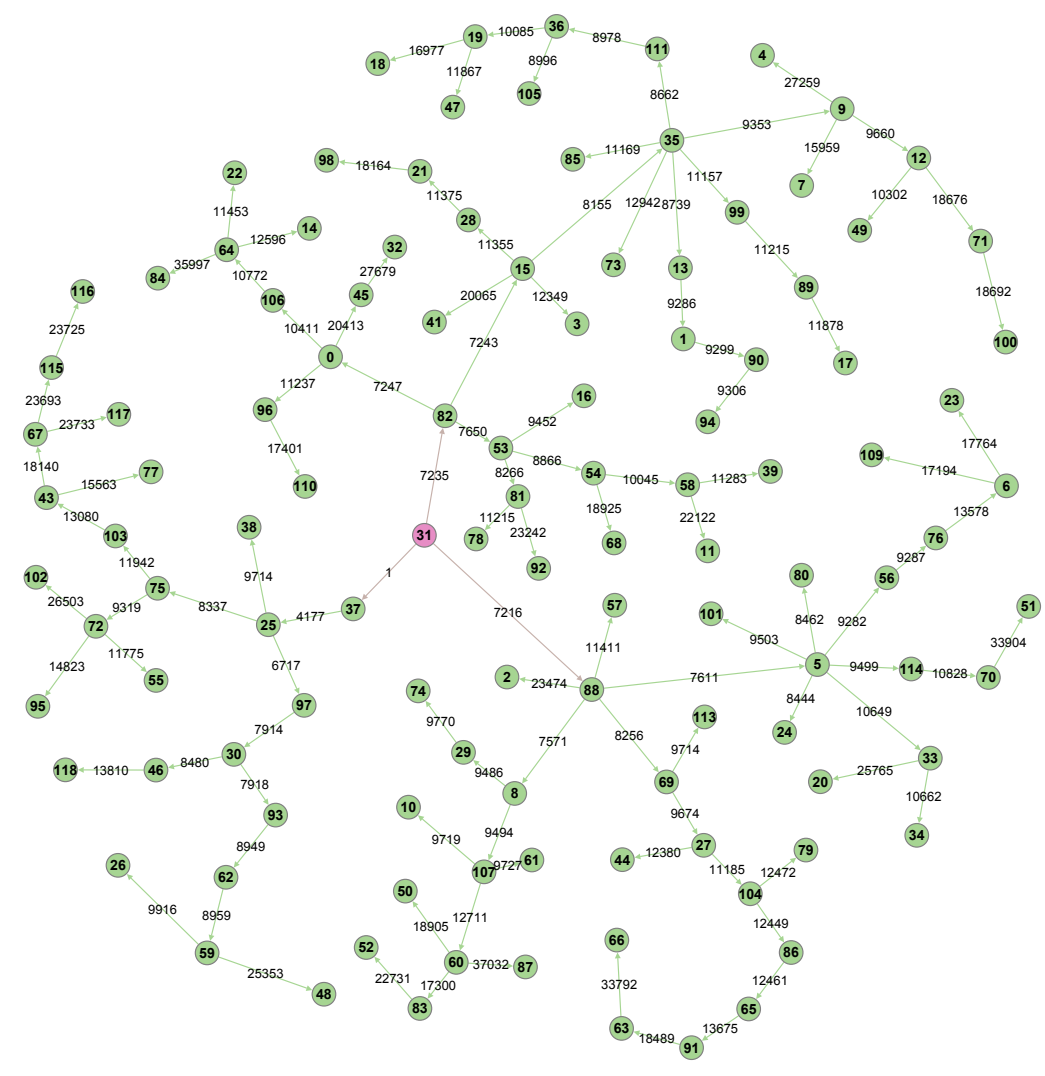

Figure 8.3: Message diffusion.

figure, each line is a transmission between two nodes showing the time when each transmission was started. Following these transmissions, the message finally arrives to all nodes (a total of 115, including the source).

We now assess the delivery success ratio. Figure 8.4 provides a graphical representation of the average delivery success ratio at intervals of 1 hour. This ratio was obtained by calculating the number of messages that are generated at a given hour $(h)$ of a day: $m s j_{s}[h]$, and the number of these messages that reach their destination $m s j_{r}[h]$; so the hourly ratio is $m s j_{r}[h] / m s j_{s}[h]$. We include only two representative plots for the smallest and biggest buffer size (50MB and $1 G B)$ with different TTLs. In the first plot (Figure 8.4a) with a TTL of $12 \mathrm{~h}$, we can see that the delivery success ratios are related to user activities, i.e. at night people are sleeping, thus their motion is reduced and the delivery probability is reduced. At daytime the motion is restored, and the delivery probability goes back to previous 


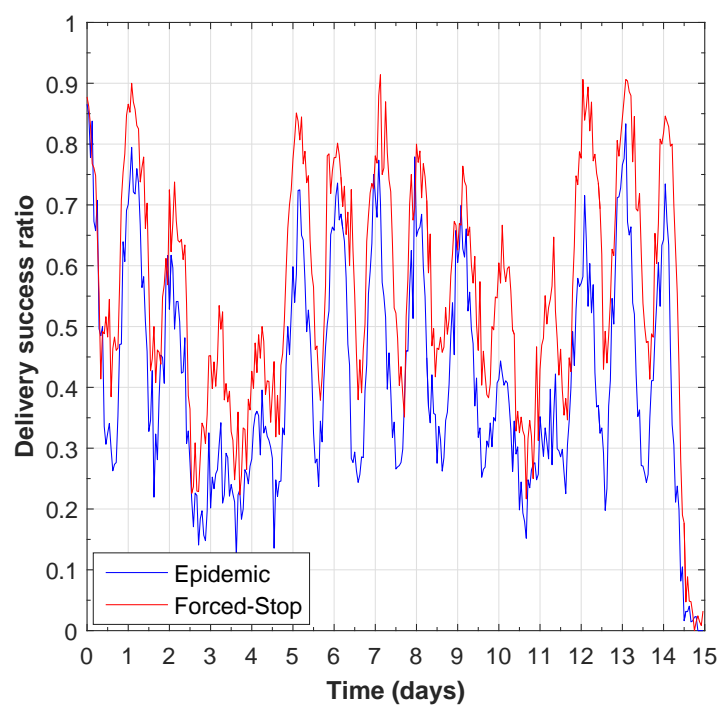

(a) Buffer size $50 \mathrm{MB}$ with TTL $=12 \mathrm{~h}$.

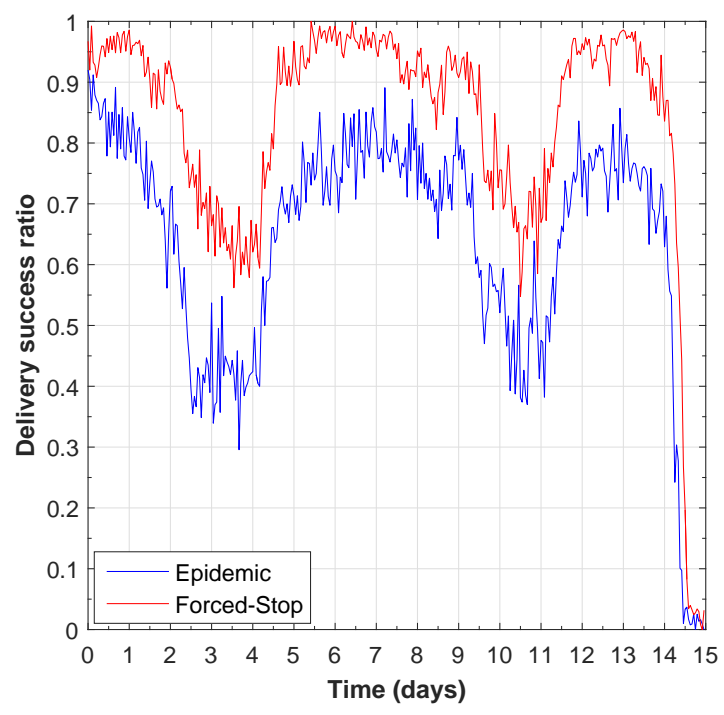

(b) Buffer size $1 \mathrm{~GB}$ with $\mathrm{TTL}=24 \mathrm{~h}$.

Figure 8.4: Average delivery success ratio by hour. 
levels. Nevertheless, when we increase the TTL to $24 \mathrm{~h}$ (and the buffer size), the results are quite different (see Figure 8.4a). In this case, due to the longer life time of messages, the daily activities are not so evident, but we can clearly see the weekly activities (for example, days 3-4 and 10-11 are weekends, so message diffusion is reduced). Regarding the effectiveness, in all cases the trend is similar, showing that Forced-Stop has a higher delivery probability than Epidemic diffusion when the rest of parameters remain the same.

Figure 8.5a shows the average delivery success ratio of all the messages, depending on different buffer sizes for the four combinations of TTL and message spreading approaches simulated. In this case, the ratio is obtained as the quotient between the total number of messages that reach their destination and the number of messages generated in the simulation. In this plot we can clearly see that, when the buffer size is bigger, or when the TTL is higher, more messages are stored in each node, improving the delivery probability. We can see that the Forced-Stop approach, as it avoids incomplete transmissions, improves the delivery probability. The Forced-Stop approach presents approximately a 30\% higher ratio than for the Epidemic protocol. This advantage is even higher for a TTL of 24 hours. The most interesting result from these experiments is that buffer size is not so determinant after a certain value, since it is sufficiently large to store most of the generated messages.

Regarding the message delivery latency, Figure 8.5b shows the average delivery time of all messages depending on buffer size, for the two different dissemination approaches and TTL times. In general, using Forced-Stop reduces the latency time in contrast to the Epidemic protocol, decreasing it by about $20 \%$ or $30 \%$ for a TTL of 12 or 24 hours. The impact of buffer size is not as dramatic, being close to $5 \%$ or $10 \%$ for each TTL, as it is the case for the delivery probability. Note also that the latency increases with the TTL, due to the improved delivery ratio, i.e., more messages reach the destination, but with greater latency.

Considering the overhead of the protocols, Figure 8.6 compares the buffer occupancy (that is, the percentage of buffer used), for both dissemination approaches. These plots show the average buffer occupancy among all nodes at each time step in the simulation. As in previous experiment, we only include two extreme cases, $50 \mathrm{MB}$ and $1 \mathrm{~GB}$ buffer size, with a TTL value of 12 and 24 hours, respectively. From these results it becomes clear that a buffer of 1GB is large enough to store all messages even for a large TTL, while a buffer of 50MB gets easily full even for a smaller TTL. In order to determine the required buffer size, we evaluate the maximum buffer occupancy for the whole simulation. Figure 8.7a plots this maximum buffer occupancy for different TTL values and dissemination approaches. As expected, buffer occupancy is higher for the Forced-Stop than for the Epidemic diffusion scheme because, as the delivery probability is higher, more messages remain alive in the whole network.

Another interesting metric, related to protocol overhead, is the amount of information forwarded per node. Similarly to the previous analysis, the results in 


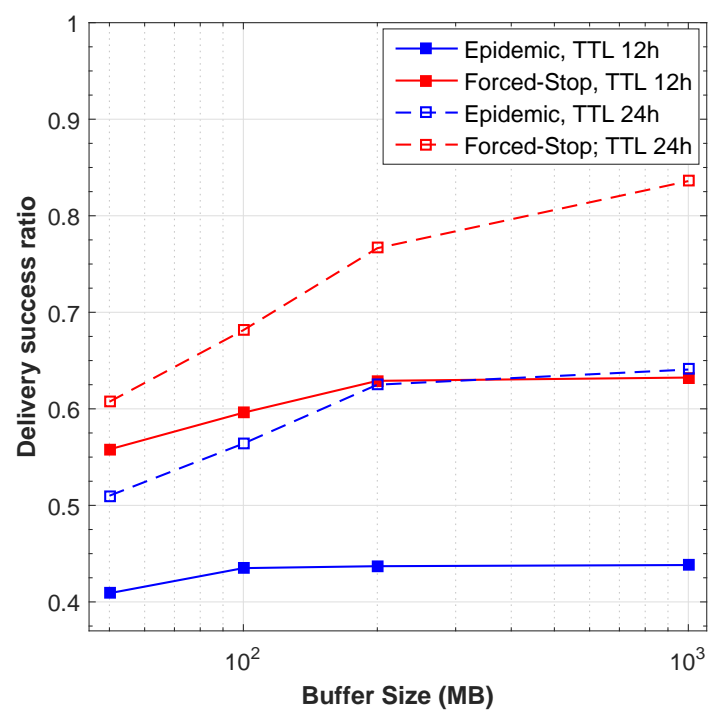

(a) Average delivery success ratio.

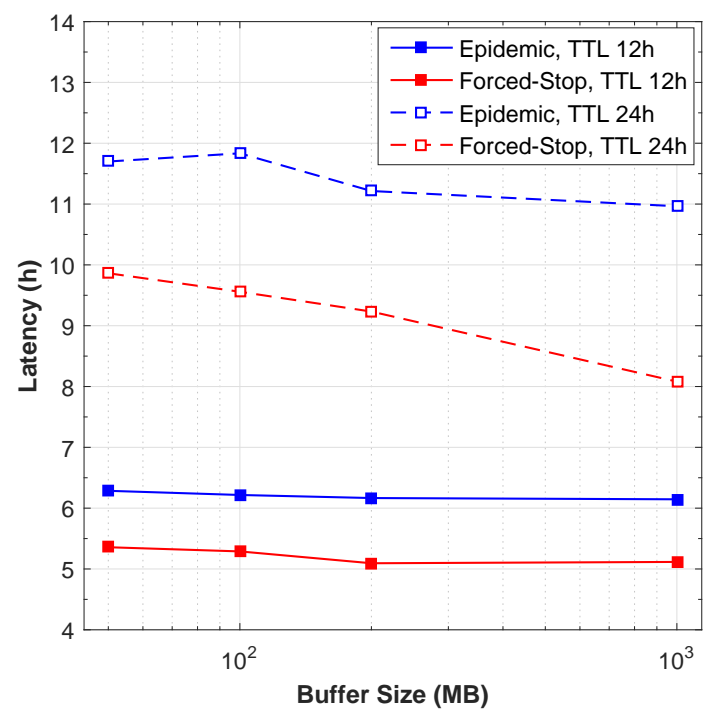

(b) Delivery latency time (in hours).

Figure 8.5: Delivery success ratio and Latency depending on buffer size (with $\mathrm{x}$-axis in $\log$ scale) 


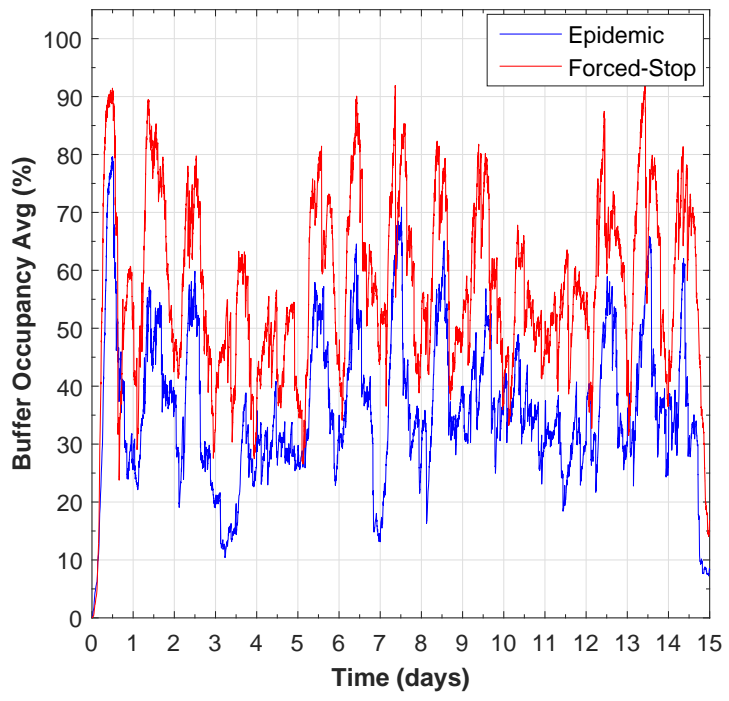

(a) Buffer size 50MB, TTL $12 \mathrm{~h}$.

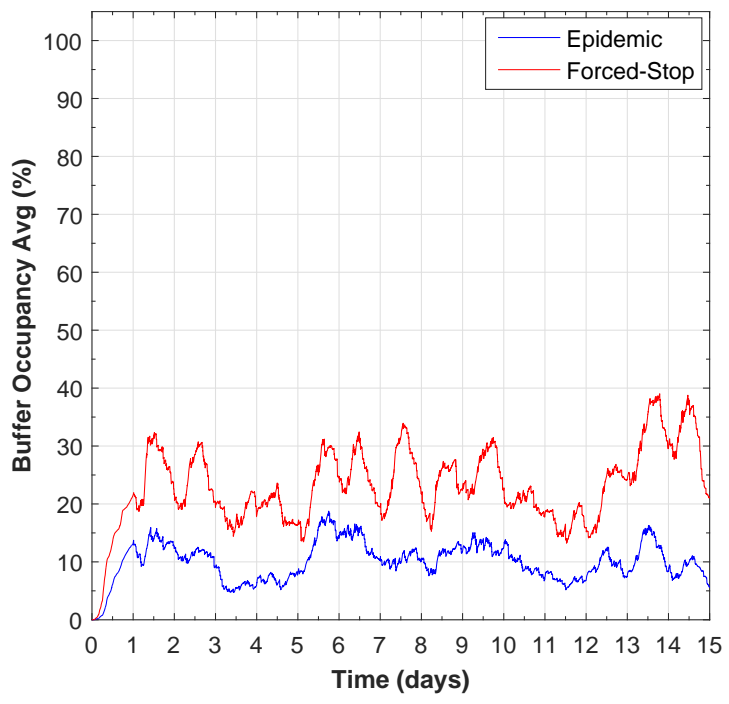

(b) Buffer size 1GB, TTL 24h.

Figure 8.6: Delivery success ratio and Latency depending on buffer size (with $\mathrm{x}$-axis in log scale) 


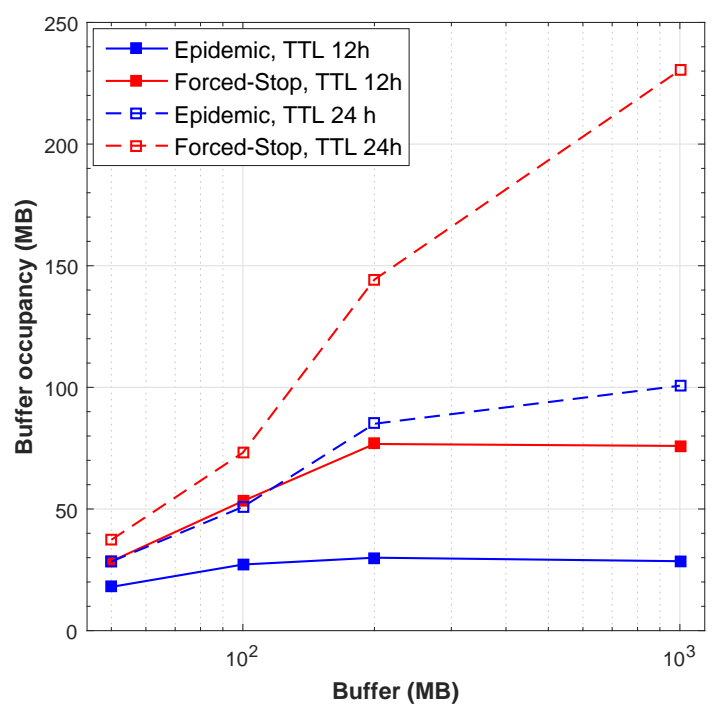

(a) Maximum buffer occupancy.

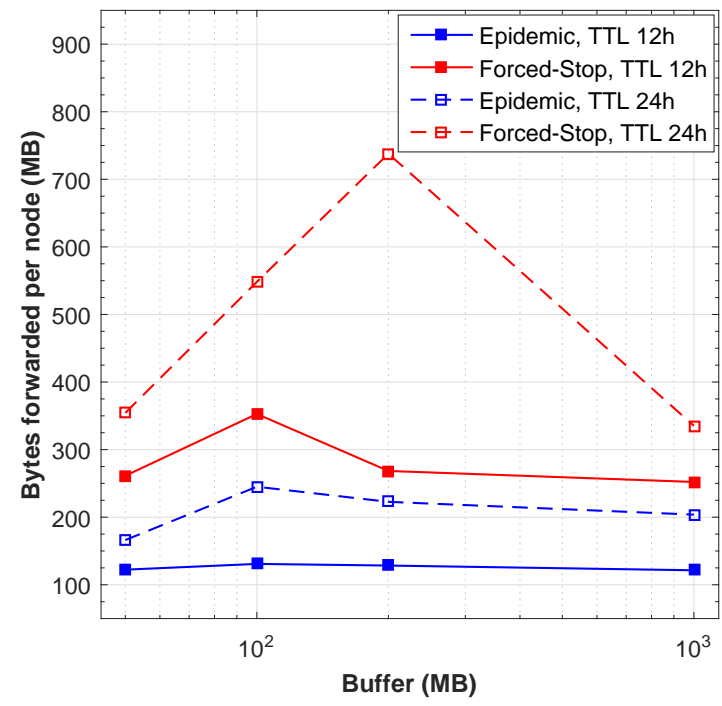

(b) Bytes daily forwarded per node.

Figure 8.7: Overhead results: Buffer occupancy and forwarded bytes 
8.4. Performance Evaluation of Forced-Stop

Table 8.2: Message statistics with a TTL of $12 \mathrm{~h}$ (x 1,000).

\begin{tabular}{|c|c|c|c|c|c|r|r|c|}
\hline $\begin{array}{c}\text { Buffer } \\
\text { size }\end{array}$ & Protocol & Created & Relayed & Aborted & Dropped & Removed & Delivered & $\begin{array}{c}\text { Delivery } \\
\text { probability }\end{array}$ \\
\hline \hline \multirow{2}{*}{$50 \mathrm{MB}$} & Epidemic & 44.2 & $2,154.9$ & 7.1 & $1,480.9$ & 696.5 & 18.1 & 0.41 \\
\cline { 2 - 9 } & Forced-Stop & 44.1 & 6.065 .2 & 0.0 & $2,665.8$ & $3,409.7$ & 24.6 & 0.56 \\
\hline \multirow{2}{*}{$100 \mathrm{MB}$} & Epidemic & 44.2 & $1,793.1$ & 7.8 & $1,677.6$ & 136,5 & 19.2 & 0.44 \\
\cline { 2 - 9 } & Forced-Stop & 44.0 & $4,967.6$ & 0.0 & $2,886.8$ & $2,089.8$ & 26,1 & 0.60 \\
\hline \multirow{2}{*}{$200 \mathrm{MB}$} & Epidemic & 44.3 & $1,690.9$ & 7,7 & $1,712.0$ & 0.0 & 19.3 & 0.44 \\
\cline { 2 - 9 } & Forced-Stop & 44.4 & $3,272.1$ & 0.0 & $3,151.6$ & 128.2 & 27.9 & 0.63 \\
\hline \multirow{2}{*}{$1 \mathrm{~GB}$} & Epidemic & 43.9 & $1,701.5$ & 7.5 & $1,722.2$ & 0.0 & 19.2 & 0.44 \\
\cline { 2 - 8 } & Forced-Stop & 44.3 & $3,166.8$ & 0.0 & $3,174.3$ & 0.0 & 28.1 & 0.63 \\
\hline
\end{tabular}

Table 8.3: Message statistics with a TTL of 24h (x 1,000).

\begin{tabular}{|c|c|c|c|c|c|r|r|c|}
\hline $\begin{array}{c}\text { Buffer } \\
\text { size }\end{array}$ & Protocol & Created & Relayed & Aborted & Dropped & Removed & Delivered & $\begin{array}{c}\text { Delivery } \\
\text { probability }\end{array}$ \\
\hline \hline \multirow{2}{*}{$50 \mathrm{MB}$} & Epidemic & 44.2 & $4,088.9$ & 7.5 & $1,525.2$ & $2,561.4$ & 22.6 & 0.51 \\
\cline { 2 - 9 } & Forced-Stop & 44.5 & $13,522.1$ & 0.0 & $2,604.5$ & $10,881.0$ & 27,0 & 0.61 \\
\hline \multirow{2}{*}{$100 \mathrm{MB}$} & Epidemic & 44.4 & $3,846.4$ & 9,5 & $1,854.7$ & $1,979.7$ & 25.1 & 0.60 \\
\cline { 2 - 9 } & Forced-Stop & 44.0 & $13,048.5$ & 0.0 & $3,064.2$ & $9,906.6$ & 29.9 & 0.68 \\
\hline \multirow{2}{*}{$200 \mathrm{MB}$} & Epidemic & 44.2 & $2,969.3$ & 9.3 & $2,385.3$ & 564.8 & 27.7 & 0.63 \\
\cline { 2 - 9 } & Forced-Stop & 44.1 & $11,137.0$ & 0.0 & $3,581.6$ & $7,444.6$ & 33.8 & 0.77 \\
\hline \multirow{2}{*}{$1 \mathrm{~GB}$} & Epidemic & 44.2 & $2,541.8$ & 9.2 & $2,513.1$ & 0.0 & 28.3 & 0.64 \\
\cline { 2 - 8 } & Forced-Stop & 44.3 & $4,183.0$ & 0.0 & $4,065.8$ & 0.0 & 37.0 & 0.84 \\
\hline
\end{tabular}

Figure $8.7 \mathrm{~b}$ are grouped by TTL. As the delivery probability increases, the amount of data delivered increases until the buffer size is big enough to hold most messages (200MB for a 12 hours TTL, and 1GB for 24 hours). This effect is caused by the dissemination process, where messages are only sent if the receiver does not have them. If the buffer is not large enough, a lot of messages are discarded to make room for new messages, and those discarded messages are sent again when nodes contact for a second time.

Finally, we present the results obtained related to the message dynamics, that is, the number of messages relayed, dropped and aborted. When mandatory stops are not enforced, transmissions will depend on the duration of contacts. If a transmission cannot be completed for the duration of a given contact, it is considered an aborted transmission. Also, a message could be dropped if the TTL expires, and removed to make room for new messages when the buffer is full. Tables 8.2 and 8.3 show the message count for all simulations considered, also showing the number of messages created, relayed and delivered. As expected, there are not aborted messages using the Forced-Stop approach, this number is comparatively small when using the Epidemic approach. Although this number is not large by any means, the effect of not losing those transmissions is quite noticeable in the delivery probability. In the Force-Stop approach there are more relayed, dropped and removed messages than in the regular Epidemic approach, simply because buffer occupancy is higher. As there are more messages in the buffers, more messages 
are transmitted, and so more message could be dropped or removed.

In this section a new diffusion method was presented, called Forced-Stop, based on controlling nodes mobility to achieve complete message transfers in opportunistic networks. Using The ONE simulator and a realistic environment based on real human mobility traces, we compared our proposal with the classical Epidemic diffusion.

The experiments showed that forcing devices to stop moving to complete the data delivery process can improve the performance of the whole diffusion process. Our diffusion model provides a higher delivery success ratio and lower delivery times at the expense of higher buffer occupation and longer transmission, and we demonstrate that forcing to stop the nodes to exchange information does improve the message dissemination ratio. In the next section this method will be improved with some control mechanisms.

\subsection{The Friendly-Sharing Approach}

Based on the results of the previous section, we can see that the Forced-Stop approach is feasible. In this section we propose an improvement of the ForcedStop approach called Friendly-Sharing. This new method adds control mechanisms and resource optimization, in order to enhance the message dissemination.

\subsubsection{Message Diffusion}

The critical factor affecting diffusion is the duration of the contact, which depends on the mobility patterns of the user. It can vary from a few seconds (a short contact in the street) to hours (for example, during a class at school), and it determines the number and the size of the messages that can be exchanged.

A way to improve the effectiveness of this diffusion is to increase the number and duration of contacts. Our proposal, called Friendly-Sharing, has to be integrated into mobile applications that will therefore ask the user to wait for some more time to extend the exchange window. The basic assumption is that installing and being interested on using such an application indicates that the user wants to actively collaborate in the diffusion of the information. Moreover, our proposal allows the applications to inform the users about how much time is needed for interchanging the messages, thus providing a friendly way to stop for a short time. We demonstrate our proposal with a proof-of-concept mobile application, called GRChat [59].

GRChat is an Android app that can establish connections among two or more phones and transmit data and images using Bluetooth. Moreover, the user can create and subscribe to groups in order to send/receive messages. GRChat works like any messaging app where the user can watch previous messages/images and write new ones. When the user pushes the Send button, GRChat connects to 

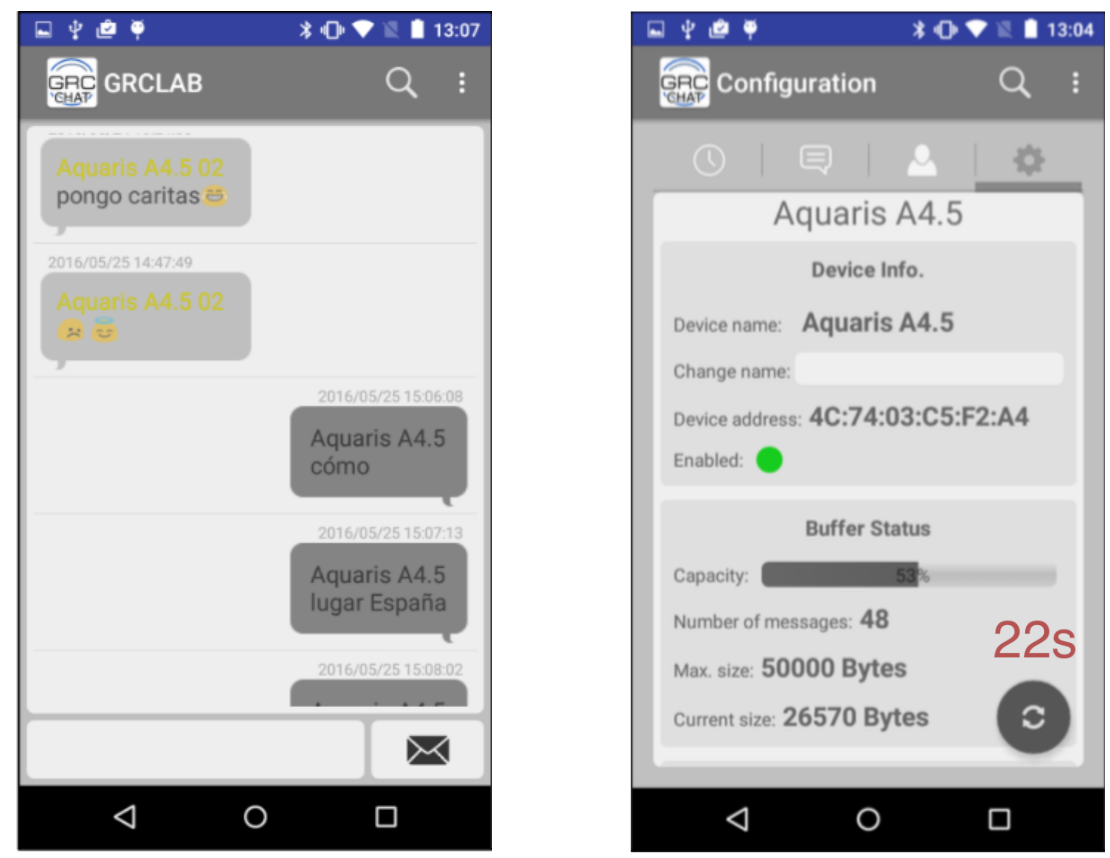

Figure 8.8: Several screenshots of the GRChat app. (a) A typical chat conversation; and (b) status of message interchange, showing the remaining time for end the transmission.

any nearby devices and sends the newly created message, as shown in Figure 8.8a; meanwhile, it also receives the new messages from the other devices. When a device gets a new message, it also tries to connect to other devices in order to complete the diffusion of the message. When no messages are sent, the application is periodically searching for nearby devices.

When the GRChat app detects a new device, it establishes a new pairwise connection and automatically starts the interchange of messages. The application notifies both users about the new connection playing a sound or vibrating. This way, the users can decide to wait some extra time in order to extend the message sharing session. The required time to complete the message interchange is shown in the application, so both users are aware of the pending time (see Figure 8.8b). This is an effective way to increase cooperation as the user knows exactly the time it should wait. Nevertheless, due to the cooperative approach, users are not forced to wait until all messages are exchanged.

We evaluate three different user behaviours:

- No-wait: The users do not stop any additional time. In this case, the number 
of messages exchanged between nodes will depend on how long they remain within the communication range and clearly on the data rate of the channel. If this contact duration is very small, no messages will be transmitted.

- Full-wait: The users wait during the time required to exchange all the messages, that is, similar to the previous Forced-Stop proposed approach. In this case, the owner of each mobile device will control this exchange by stopping and waiting until the message transmission is fully completed.

- Finite-wait: To avoid interfering too much with the user mobility, users wait only for some extra time to send and receive some of the pending messages.

\subsubsection{Buffer Management}

It is important to select the order of the messages sent when two nodes are sharing their messages, especially when users decide not to wait until all messages are sent. Furthermore, we must be aware that both the buffer size and the channel bandwidth are limited. It is, therefore, important to define mechanisms to optimize the use of these resources in a mobile device. In this subsection, in order to evaluate the impact of the forwarding methods on the messages delivery probability and latency, we describe some approaches related to the message forwarding and dropping process. The buffer management was deeply explained and tested in the section 4. As a short summary, we considered four different strategies (see Figure 4.1) for each of the two main buffer management activities, namely:

- Message forwarding: i.e., the criteria for selecting messages from the buffer for transmission when two nodes connect: Random, FIFO, Oldest (send lowest TTL message first) and Smallest (send small messages first).

- Message dropping: i.e., the criteria for dropping messages from the buffer when it is full: Random, FIFO, Oldest (remove lowest TTL message first) and Largest (remove large messages first).

\subsection{Performance Evaluation of Friendly-Sharing}

In this section, we evaluate the efficiency of our proposed Friendly-Sharing approach. As commented previously, the performance of the message diffusion will mainly depend on the mobility of the users and the number of contacts and their duration between pair of users. The impact of the dropping and forwarding policies in the local buffer is also evaluated, in order to select the best policy.

We employed The ONE simulator using the same real movement trace of mobile users of the last section. This trace comes from an experiment at the NCCU campus [154], where GPS position data was collected during two weeks (336 hours) using an Android app installed in the smartphones of 115 students. 
The workload considered tried to mimic the typical data-flow for a multimedia messaging application where shorter messages are far more common than larger one. Similar to previous experiment, three message sizes and frequencies were considered: (1) a short text message $(1 \mathrm{kB})$ every hour; $(2)$ a photo $(1 \mathrm{MB})$ every $18 \mathrm{~h}$; and (3) a video or high-resolution picture (10MB) every $96 \mathrm{~h}$. These frequencies were based on the statistics of WhatsApp (Facebook, Inc.) message usage from [135], while sizes are approximations of the content produced by current mobile phone hardware.

The communication range $(r)$ was set to $7.5 \mathrm{~m}$ with bandwidth $B_{w}=2.1 \mathrm{Mb} / \mathrm{s}$. These assumptions allow taking into consideration the necessary set-up time of connections and the possible impact of interferences. These values are based on the Bluetooth 2.0 class 2 specifications. Other technologies such as WiFi-Direct are not considered to avoid issues like disconnecting the device from the internet. WiFi is increasingly being used for data connectivity, especially at large enterprise areas or university campuses, and it would be a nuisance for users to deal with these disconnection periods.

\subsubsection{Trace Characterization}

In order to characterize mobility and determine the structure of the contacts, we analyzed the trace used in the experiments. From this study, we could determine some social patterns of the trace that are useful to understand the performance of the Friendly-Sharing approach.

Figure 8.9 shows the contact graph for different time intervals, starting from the first $3 \mathrm{~h}$ of the trace up to $24 \mathrm{~h}$. Each node is labelled by a number and the diameter of each circle is proportional to the number of contacts. Node groups are represented by different colours and were computed using the FruchtermanReingold algorithm [47]. We can see that the social interrelation given by the dynamics of the contacts numbers increases according to the simulation time, while the number of communities decreases: on the first $3 \mathrm{~h}$ of simulations, the number of communities are 12 , for $6 \mathrm{~h}$ and $12 \mathrm{~h}$, we have eight communities, and, finally, after $24 \mathrm{~h}$, we only have six communities. This means that the number of "communities" slowly decreases, thus contributing to message diffusion and all nodes eventually getting connected. This implies increasing the message TTL to $12 \mathrm{~h}$ or $24 \mathrm{~h}$ in order to produce a better delivery rate.

Figure 8.10 presents the average node speed at each hour of the movement trace, clearly showing that nodes have very dissimilar movement patterns. Some nodes move frequently and with considerable speed, while other nodes are quite static and move with lower speed. This effect could be explained by the students place of residence, e.g., some sleep close to the campus (or even inside) while others have to travel further away to their homes.

Although nodes without movement in a large period of time could be considered not relevant for message interchanges, Figure 8.11 shows exactly the opposite. In 


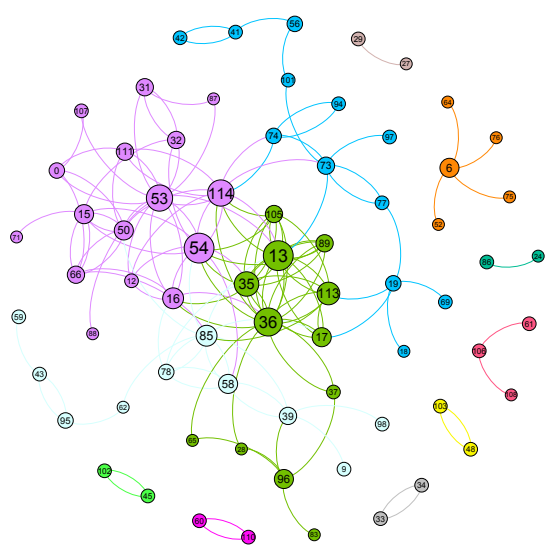

(a) $3 \mathrm{~h}$.

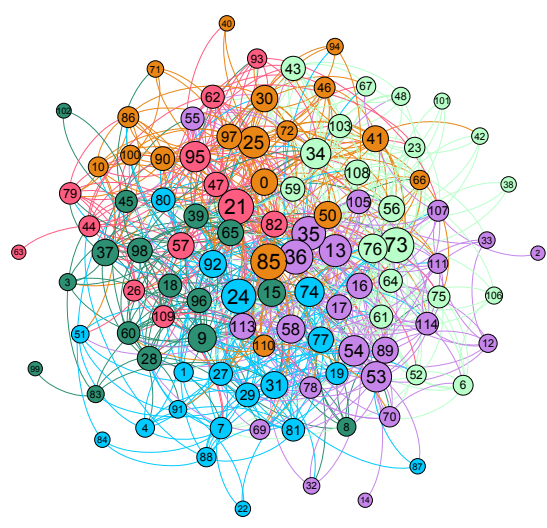

(c) $12 \mathrm{~h}$.

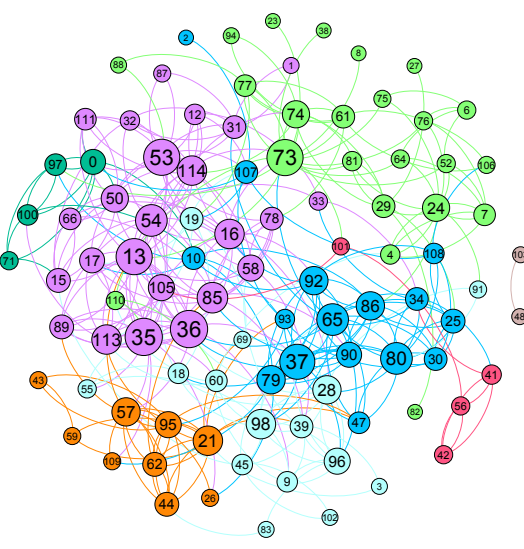

(b) $6 \mathrm{~h}$.

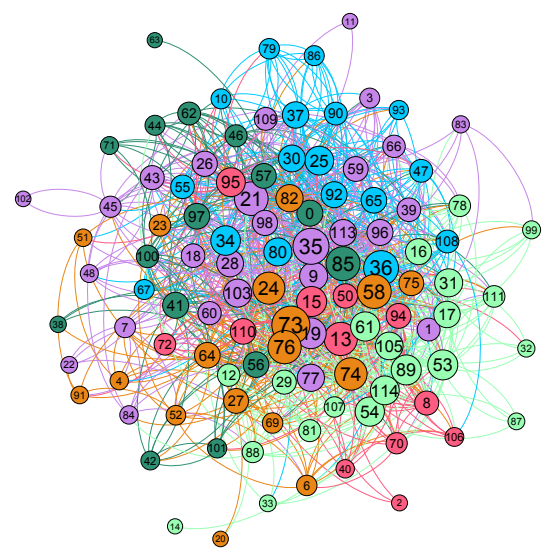

(d) $24 \mathrm{~h}$.

Figure 8.9: Contact graph for different time intervals of the trace: 3 (a), 6 (b), $12(\mathrm{c})$, and $24(\mathrm{~d})$ hours. 


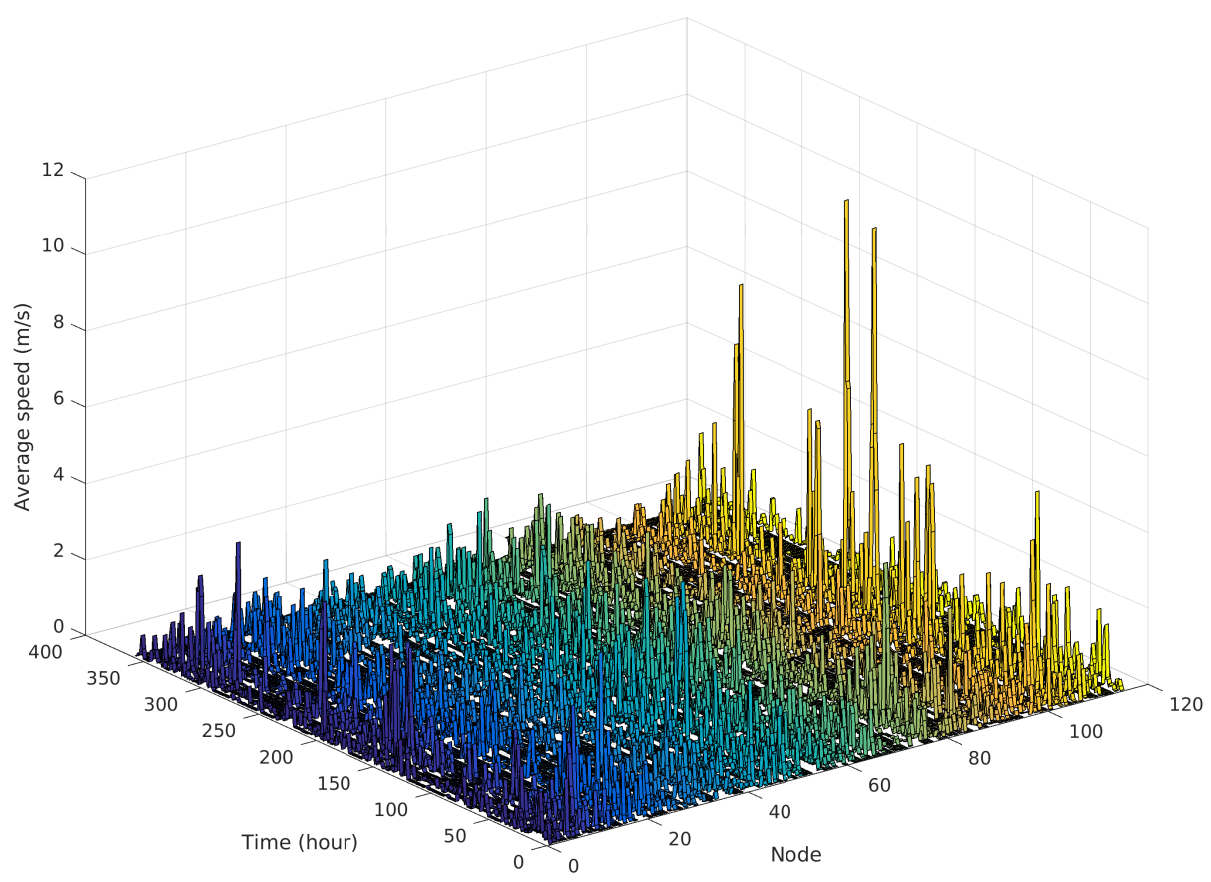

Figure 8.10: Average node speed at each hour of the movement trace.

this plot, the $x$-axis shows the average speed computed as in Figure 8.10, while the $y$-axis shows how much time a node is in contact during an hour. For example, a node that has an average speed of $2 \mathrm{~m} / \mathrm{s}$ and a total contact duration of $1 \mathrm{~h}$ across all contacts will be plotted in position $(2,1)$. As a node could be contacted by more than one node at the same time, the $y$-axis extends away from 1 . This inverse correlation between movements and contacts is easily explained by the students' typical behaviour: they stay together in a class for a bounded amount of time, they move a small distance to the next class, and so on. Even while not attending classes, they spend time with other students in the cafeteria, library, or other campus services.

\subsubsection{Buffer Management Evaluation}

In this subsection, we evaluate the different buffer management policies for different buffer sizes, in order to select the best combination. Several simulations were executed for all the 16 possible combinations using the Epidemic protocol. Each forwarding and dropping combination was tested with a message TTL of 


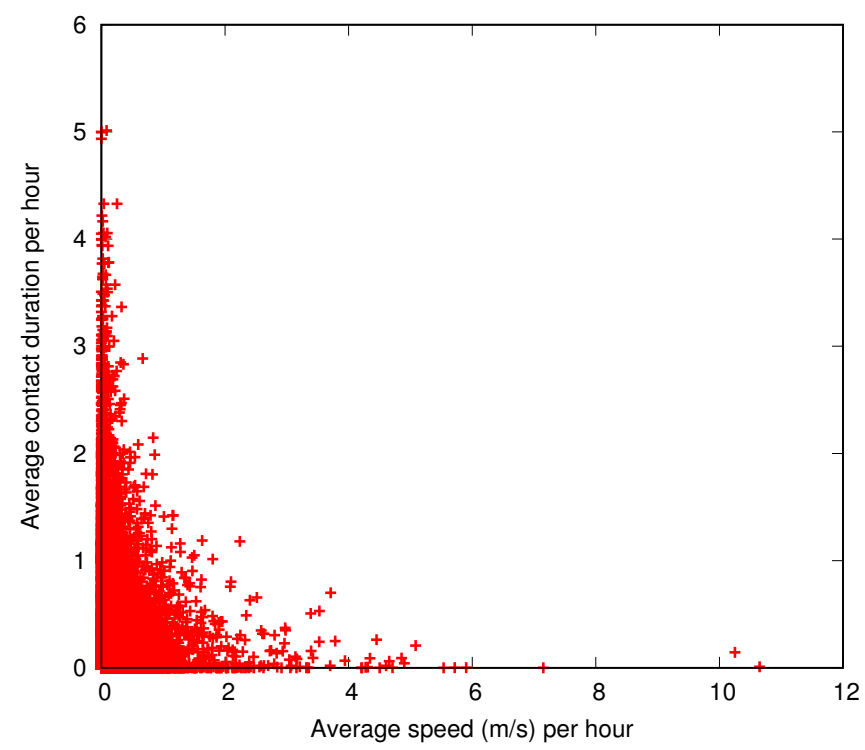

Figure 8.11: Average speed and number of contacts for all nodes at each hour of the movement trace.

Table 8.4: Simulation parameters varied to evaluate queue management policies.

\begin{tabular}{|c|c|}
\hline Parameter & Values \\
\hline \hline Buffer Size & $50 \mathrm{MB}, 100 \mathrm{MB}, 200 \mathrm{MB}, 1 \mathrm{~GB}$ \\
\hline Time to Live & $12 \mathrm{~h}, 24 \mathrm{~h}$ \\
\hline Forward policy & Random, FIFO, Oldest, Smallest \\
\hline Discard policy & Random, FIFO, Oldest, Largest \\
\hline
\end{tabular}

12 or $24 \mathrm{~h}$, and buffer sizes of $50 \mathrm{MB}, 100 \mathrm{MB}, 200 \mathrm{MB}$ and $1 \mathrm{~GB}$. These simulation parameters are summarized in Table 8.4.

Figures $8.12 \mathrm{a}$ and $8.12 \mathrm{~b}$ show the relationship between delivery probability and average message latency for each of the simulations. This plot uses a point for each buffer management policy combination with the same symbol for a particular buffer size. It shows that there is a clear correlation between delivery probability and latency and that, as the buffer size increases, the variability of the delivery probability decreases. This last fact is even more evident when increasing the TTL message, where a minimum of a $200 \mathrm{MB}$ buffer is required to stabilize this metric. 


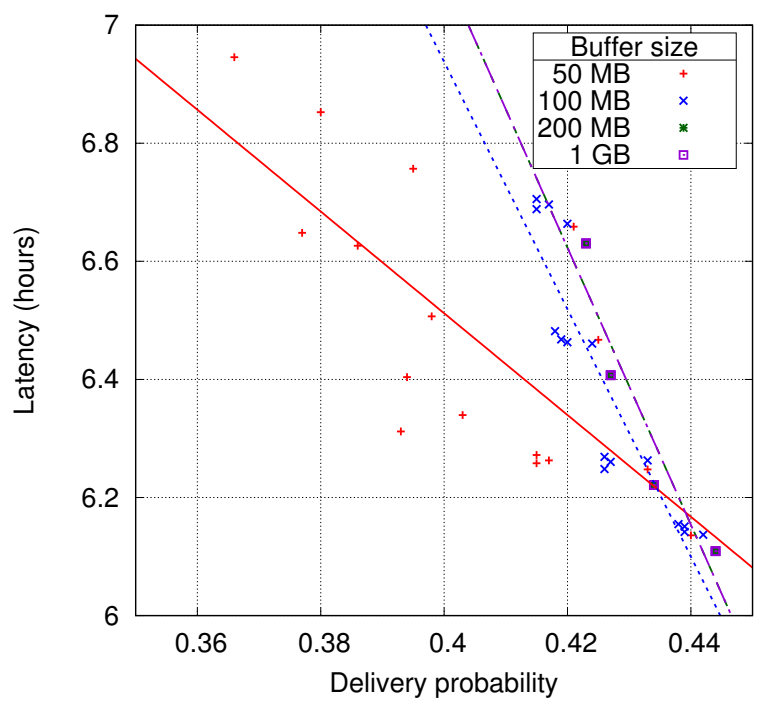

(a) $12 \mathrm{~h}$ case.

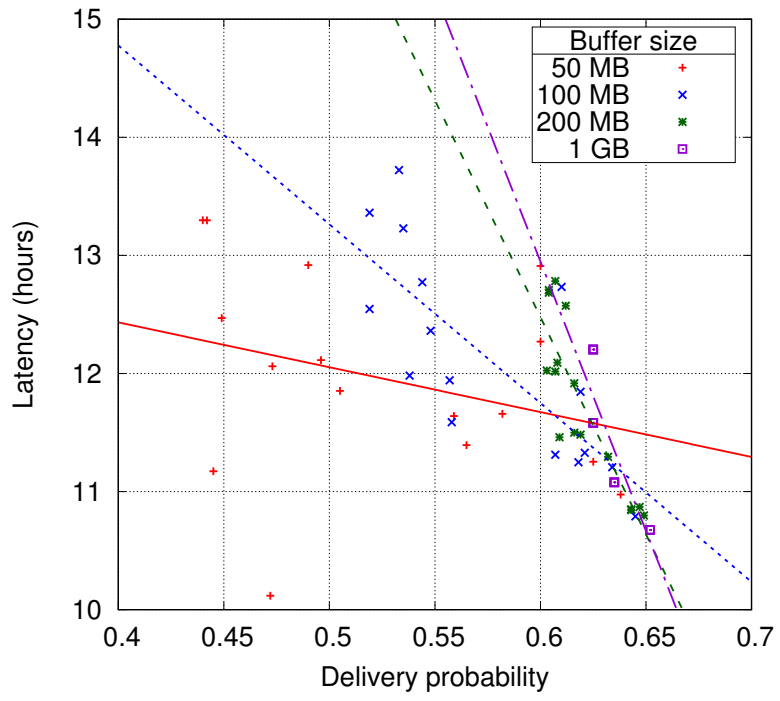

(b) $24 h$ case.

Figure 8.12: Delivery probability versus latency for a 12 and 24h TTL (Time To Live) with different buffer sizes and queue policies. 
Table 8.5: Delivery probability for a 12 hour TTL with different buffer sizes and queue policies.

\begin{tabular}{|c|c|r|r|r|r|}
\hline \multirow{2}{*}{$\begin{array}{c}\text { Buffer } \\
\text { size }\end{array}$} & Drop & \multicolumn{4}{|c|}{ Forwarding policy } \\
\cline { 3 - 6 } & policy & Random & FIFO & TTL & Smallest \\
\hline \hline \multirow{4}{*}{$50 \mathrm{MB}$} & Random & 0.394 & 0.386 & 0.380 & 0.415 \\
\cline { 2 - 6 } & FIFO & 0.403 & 0.398 & 0.395 & 0.415 \\
\cline { 2 - 6 } & TTL & 0.393 & 0.377 & 0.366 & 0.417 \\
\cline { 2 - 6 } & Largest & 0.433 & 0.425 & 0.421 & $\mathbf{0 . 4 4 0}$ \\
\hline & Random & 0.426 & 0.419 & 0.415 & 0.439 \\
\cline { 2 - 6 } $100 \mathrm{MB}$ & FIFO & 0.427 & 0.420 & 0.417 & 0.438 \\
\cline { 2 - 6 } & TTL & 0.426 & 0.418 & 0.415 & 0.439 \\
\cline { 2 - 6 } & Largest & 0.433 & 0.424 & 0.420 & $\mathbf{0 . 4 4 2}$ \\
\hline $200 \mathrm{MB}$ & Any & 0.434 & 0.427 & 0.423 & $\mathbf{0 . 4 4 4}$ \\
\hline $1 \mathrm{~GB}$ & Any & 0.434 & 0.427 & 0.423 & $\mathbf{0 . 4 4 4}$ \\
\hline
\end{tabular}

The main factor for a good delivery probability is how long messages are available in the network (TTL), for larger times, the probability of arrival increases. The buffer size also plays an important role, as the buffer size increases the buffer management policies have a smaller impact on the message delivery. When the buffer size is large enough to keep almost all messages, the dropping policy is no longer relevant. Tables 8.5 and 8.6 show the delivery probability for each policy combination, where the best values are for the combination of smallest forwarding and largest dropping policies. In this case, the buffer management policies are giving priority to the small messages, which also have a better transmission chance in a short contact time.

\subsubsection{Friendly-Sharing Diffusion Evaluation}

In this section, we study the benefits of the proposed Friendly-Sharing diffusion approach compared to the standard Epidemic protocol. For the sake of brevity, we will focus on the smallest/largest buffer management policies as the previous subsection demonstrates that they obtain the best delivery probability. We repeat the same simulation parameters schema with different buffer sizes and TTL times, summarized in Table 8.7.

Figure 8.13 a shows the delivery ratio of opportunistic networks in relation to the Friendly-Sharing maximum time. A value of 0 in the $x$-axis represents the Epidemic protocol without any additional wait, and a value of $\infty$ refers to the full-wait approach, where nodes wait until they transmit all messages in their buffers. The experiment shows, as expected, that waiting until all messages are 


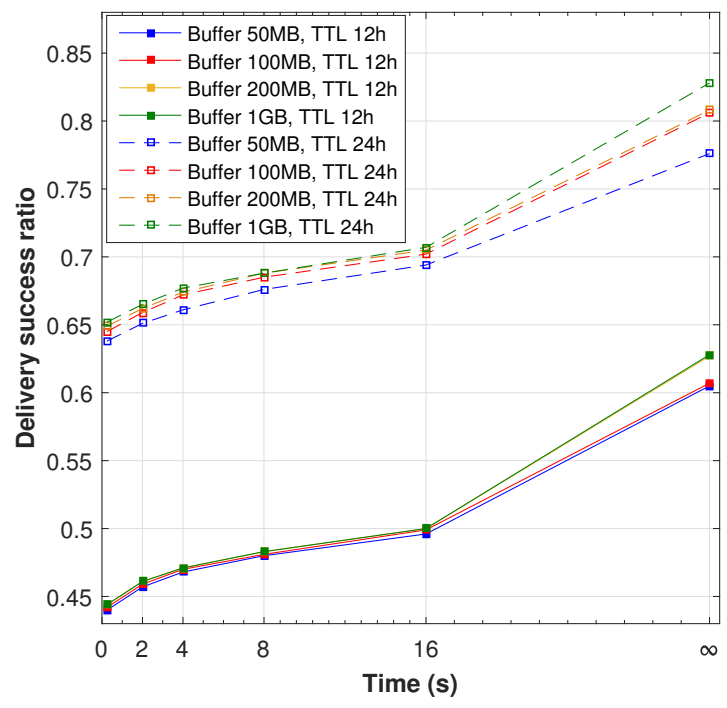

(a) Delivery probability.

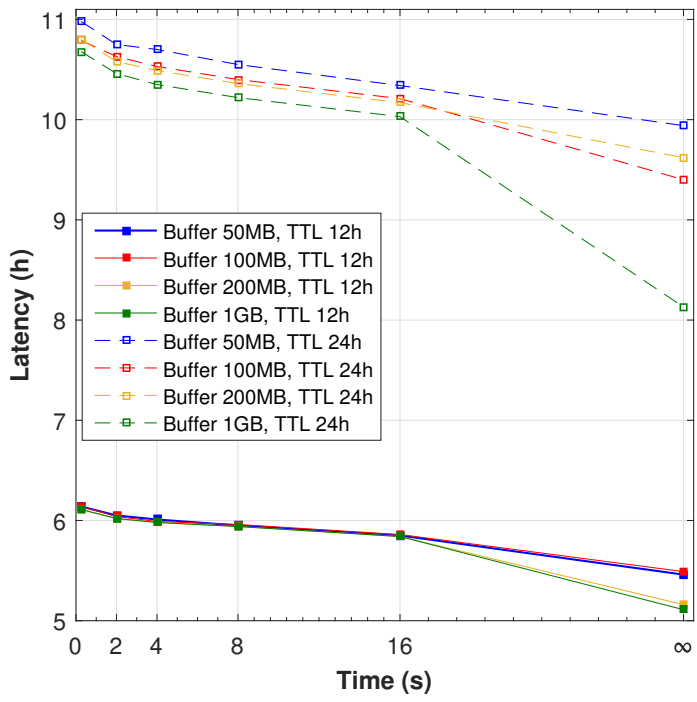

(b) Latency time (hours).

Figure 8.13: Average delivery success ratio and latency. 
Table 8.6: Delivery probability for a 24 hour TTL with different buffer sizes and queue policies.

\begin{tabular}{|c|c|r|r|r|r|}
\hline \multirow{2}{*}{$\begin{array}{c}\text { Buffer } \\
\text { size }\end{array}$} & $\begin{array}{c}\text { Drop } \\
\text { policy }\end{array}$ & \multicolumn{4}{|c|}{ Forwarding policy } \\
\cline { 3 - 6 } & Random & FIFO & TTL & Smallest \\
\hline \hline \multirow{4}{*}{$50 \mathrm{MB}$} & Random & 0.473 & 0.449 & 0.442 & 0.559 \\
\cline { 2 - 6 } & FIFO & 0.505 & 0.496 & 0.490 & 0.565 \\
\cline { 2 - 6 } & TTL & 0.472 & 0.445 & 0.440 & 0.582 \\
\cline { 2 - 6 } & Largest & 0.625 & 0.600 & 0.600 & $\mathbf{0 . 6 3 8}$ \\
\hline \multirow{4}{*}{$100 \mathrm{MB}$} & Random & 0.538 & 0.519 & 0.519 & 0.607 \\
\cline { 2 - 6 } & FIFO & 0.557 & 0.548 & 0.535 & 0.618 \\
\cline { 2 - 6 } & TTL & 0.558 & 0.544 & 0.533 & 0.621 \\
\cline { 2 - 6 } $200 \mathrm{MB}$ & Largest & 0.634 & 0.619 & 0.610 & $\mathbf{0 . 6 4 5}$ \\
\hline & Random & 0.609 & 0.603 & 0.604 & 0.643 \\
\cline { 2 - 6 } & FIFO & 0.616 & 0.607 & 0.604 & 0.643 \\
\cline { 2 - 6 } & TTL & 0.619 & 0.608 & 0.607 & 0.647 \\
\cline { 2 - 6 } & Largest & 0.632 & 0.616 & 0.612 & $\mathbf{0 . 6 4 9}$ \\
\hline $1 \mathrm{~GB}$ & Any & 0.635 & 0.625 & 0.625 & $\mathbf{0 . 6 5 2}$ \\
\hline
\end{tabular}

Table 8.7: Simulation parameters varied to evaluate wait time.

\begin{tabular}{|c|c|}
\hline Parameter & Values \\
\hline \hline Buffer Size & 50MB, 100MB, 200MB, 1GB \\
\hline Routing & Epidemic, Friendly-Sharing \\
\hline Maximum wait time & $2 \mathrm{~s}, 4 \mathrm{~s}, 8 \mathrm{~s}, 16 \mathrm{~s}, \infty$ \\
\hline Time to Live & $12 \mathrm{~h}, 24 \mathrm{~h}$ \\
\hline
\end{tabular}

interchanged improves the message diffusion. In this case, the Epidemic protocol has a delivery probability less than 0.45 , while increasing the wait time from $2 s$ to $16 s$ the delivery probability improves to 0.50 . The best probability appears for the full-wait. As in the previous experiments, the TTL parameter is the main delivery performance factor, showing that an increase from 12 to $24 h$ improves the delivery probability by about $20 \%$. Using a bigger buffer has little impact with a TTL of $12 h$ and no discernible improvement when the TTL is configured to $24 h$.

Regarding latency, comparing both plots in Figure 8.13b, it is clear how it decreases as the delivery probability increases for each TTL value. This validates our previous assumption of an approximate inverse relationship between the two measurements. Following this relationship, the buffer size does not have much 
impact on latency, and the TTL continues to be the main factor.

These experiments show a clear trade-off between delivery probability and latency. Allowing a large TTL greatly improves the delivery probability, it also increases the latency. Roughly, doubling the TTL from 12 to $24 \mathrm{~h}$ improves the delivery probability by $50 \%$, but the latency is also doubled. This limit on the routing performance is due to the movement traces themselves, which dictates how much time it takes to contact, from one node to another.

Considering protocol overhead, Figure 8.14a shows the maximum of the average buffer occupancy from each node. With the simulated workload, a buffer of around 80 and $175 \mathrm{MB}$ seems sufficient for a TTL of 12 and 24h, respectively. Bigger buffers are only required when simulating the ideal scenario with indefinite pauses. It is interesting to note that even small additional waiting times improve the delivery probability and latency without increasing the buffer overhead.

In Epidemic protocol, another overhead measurement is the amount of information forwarded per node. Figure $8.14 \mathrm{~b}$ shows the daily average bytes forwarded by each node, which is the sum of all bytes transmitted divided by the number of nodes and the simulation length in days. The number of transmissions increases greatly with a large TTL time, basically because more messages stay in the buffers during more time. A big buffer avoids unnecessary retransmissions; therefore, it also reduces the time required by nodes to stop waiting to complete the data transfer. This protocol does not provide any reception acknowledgements; therefore, nodes keep forwarding messages that have already reached their destination, up to the maximum of TTL time.

\subsubsection{Probability of User Stopping}

Similarly to the previous buffer occupancy analysis, it is interesting to point out that even making small movement pauses with our Friendly-Sharing improves the delivery probability and latency without much transmission overhead.

In our previous analysis, a full cooperation is assumed, since, as indicated before, we suppose that installing the application shows that the users are willing to follow its indications. As reasonable as this argument could be, there are cases where users may not want to stop or simply cannot stop. Furthermore, users will stop longer when contacting other related users or depending on past interactions; that is, stopping probability and duration has a strong social dependence. However, no social data can be easily extracted from the movement traces. We reflect this situation through the probability of stopping $\left(p_{s}\right)$. This probability ranges from 1, which reflects full cooperation of both users (as considered in the previous evaluations) to 0 , indicating no cooperation (and no waiting time, which is the basic Epidemic protocol).

In order to evaluate the impact of this probability, we set the buffer size to $200 \mathrm{MB}$ considering $16 \mathrm{~s}$ maximum stop time for both TTL (which is one of the experiments represented in Figure 8.13). We evaluated the delivery success ratio 

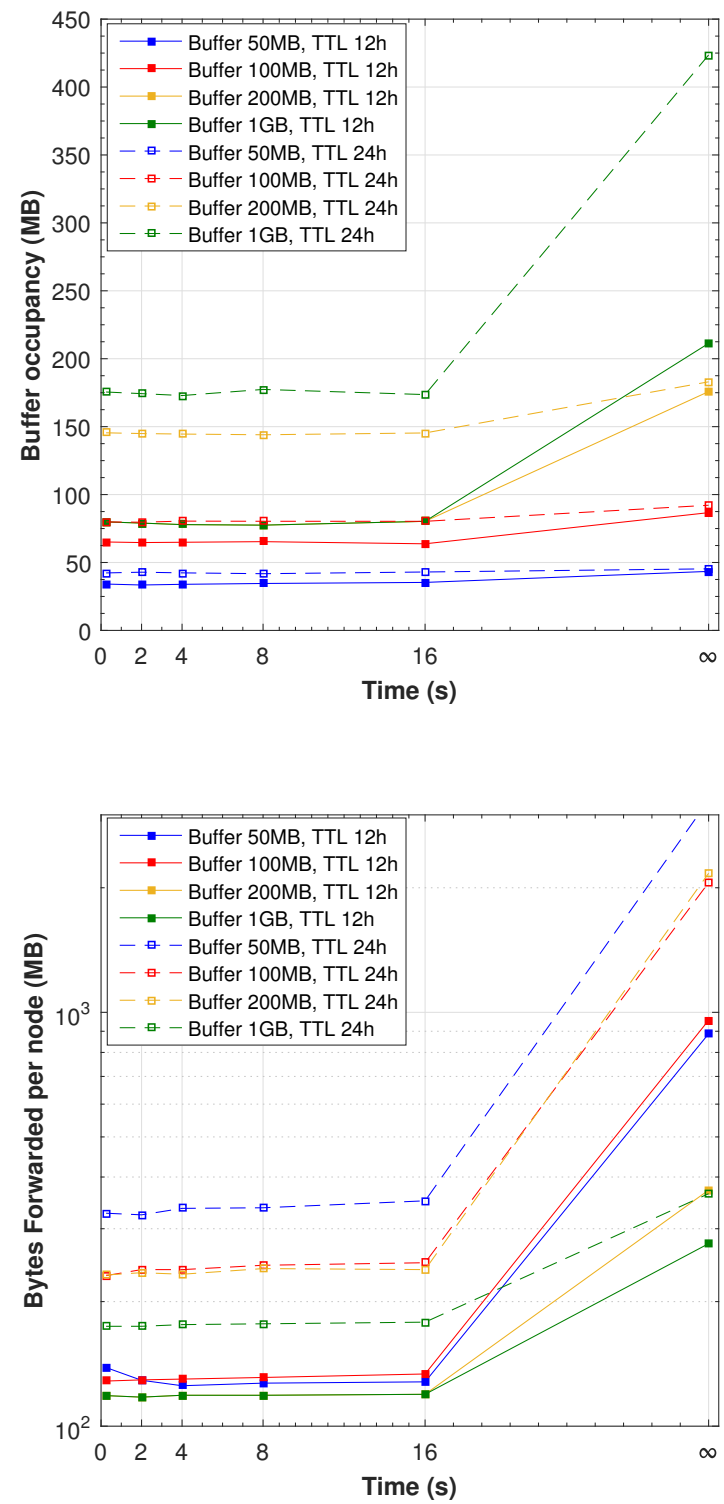

Figure 8.14: Overhead results: Buffer occupancy and forwarded bytes. (a) maximum of the average buffer occupancy from each node; and (b) average bytes daily forwarded per node ( $y$-axis in log scale). 

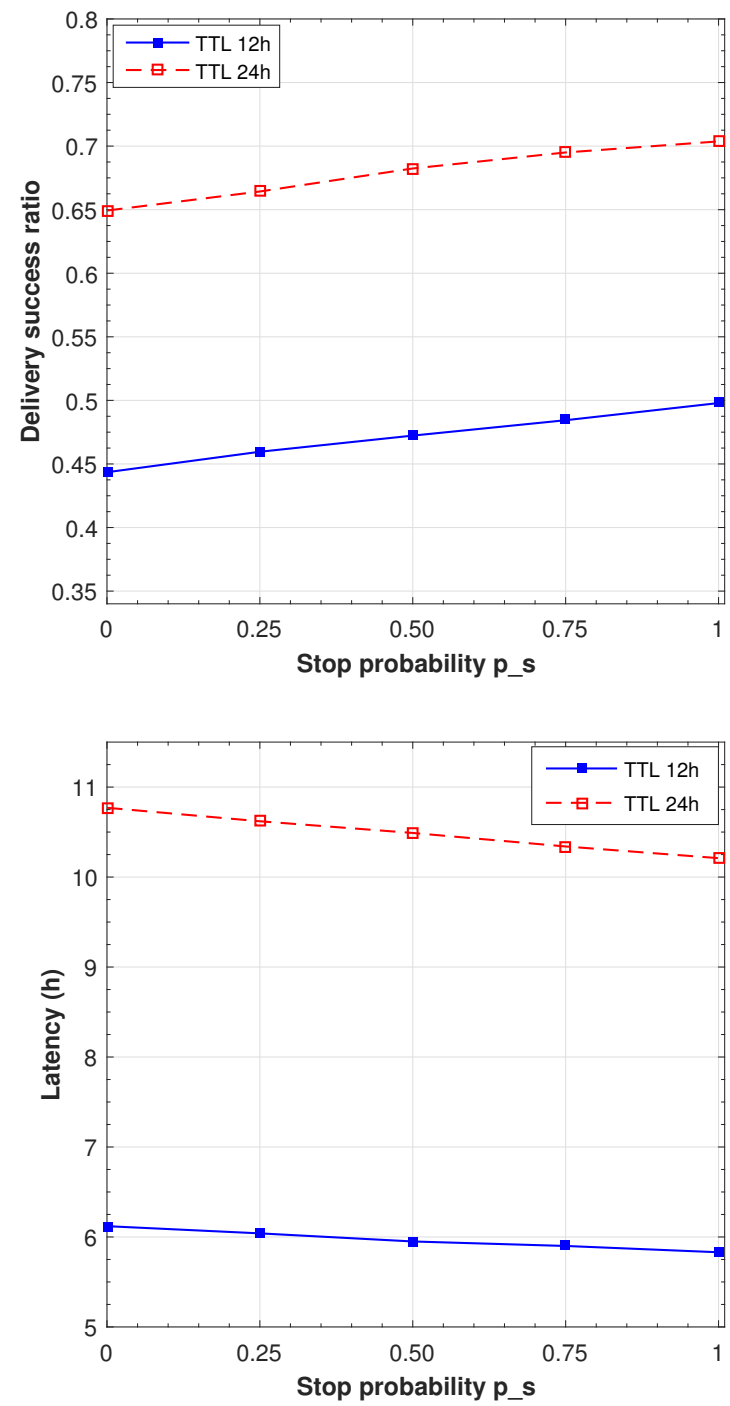

Figure 8.15: Delivery ratio (left) and latency (right) for different contact stop probabilities (16s max. stop). 
and latency for different values of the stop probability. This probability is modelled as a uniform distributed random variable independent for each contact. The results are presented in Figure 8.15, showing a linear correlation between stop probability and delivery success and latency. Moreover, we can see that stopping $16 s$ with a stopping probability of 0.5 , we obtain a similar delivery and latency performance as with a $8 s$ unconditional stop, as displayed in Figure 8.13. A similar result can be observed for a probability of 0.25 , which corresponds to $4 s$, and for a probability of 0.75 , that corresponds to $16 \mathrm{~s}$. We can, therefore, conclude that the effect of the stop probability is equivalent to reducing the waiting time by a factor $p_{s}$.

\subsection{Summary}

In this chapter, we first introduced the Forced-Stop approach as a way to improve the message diffusion. This approach was improved in the so called FriendlySharing where users are informed about the remaining time needed to interchange the messages stored in their buffers. In this way, the user can decide to wait longer in order to increase the number of messages exchanged, thus increasing the delivery rate. We used The ONE simulator with real human mobility traces and realistic message generation patterns based on social networking applications to evaluate the performance of the Friendly-Sharing diffusion. The behaviour of students produced interesting contact patterns: nodes with low mobility have more contacts than high mobility ones.

We showed that the greater the number of messages in local buffers, the better the diffusion (in terms of delivery probability and latency). Thus, a key aspect for efficiency is the buffer management policy, especially when there is no full message interchange. The experiments show that the best results were obtained when forwarding the smallest messages first and dropping the biggest ones to make room for incoming transmissions.

Regarding the waiting time for Friendly-Sharing, we also determined that when nodes wait even an extra two seconds each time they contact, it improves the delivery performance while not having a relevant impact on the buffer occupancy or on the protocol overhead.

As a proof-of-concept, we showed that Friendly-Sharing can be easily introduced in messaging applications and can effectively improve the collaboration, increasing the delivery ratio and reducing the latency of message delivery with a limited impact on buffer utilization. 


\section{Chapter 9}

\section{Conclusions, Publications, and Future Work}

\subsection{Conclusions}

As we stated along this thesis, Opportunistic wireless networks are an alternative to be considered where the Internet infrastructure is not available or it is saturated.

The main goal of this thesis was to make a depth evaluation of the main factors that impact on the message diffusion performance. Based on these results we proposed a feasible and applicable method to improve the message dissemination under epidemic diffusion environment. In detail, the main conclusions of this thesis are:

- Message diffusion performance depends mainly on the device mobility pattern and the message transmission time. This was confirmed, first by the simulations performed using different mobility traces and scenarios.

- Regarding buffer management, it can be concluded that, the greater the number of messages in local buffers, the better the diffusion is (in terms of delivery probability and latency). To keep a large number of messages not only a large buffer size is relevant but also the adequate buffer management policy. The best performance is achieved when forwarding the smallest messages first and dropping the biggest ones to make room for incoming transmissions. The experiments show a clear trade-off between delivery probability and latency. Approximately, doubling the TTL from 12 
to 24 hours improves the delivery probability by $50 \%$, but the latency is also doubled.

- Power consumption in an opportunistic network is moderately low, making feasible and recommendable the deployment of applications of this communications environment. Furthermore, it can be concluded, that in opportunistic networking, the impact on mobile device batteries is higher since the application must be running permanently. And when the mobile devices are in a coverage zone, the power consumption is greater than when it is sending information. This is a consequence of the periodical swapping of protocol control information.

- Increasing the transmission range improves significantly the message delivery ratio. That is, in the specific case of using sub-gigahertz wireless technologies, in our case the novel Long Range (LoRa) technology in a opportunistic network using the Epidemic protocol. LoRa improves significantly the message delivery ratio over WiFi in the range of about $40 \%$ to $50 \%$ for TTLs of 12 and 6 hours respectively. This is because a wider communication range not only allows more contacts but also those contacts will have greater durations.

- Fixed nodes only increase the overhead while mobile nodes boost the performance significantly. We evaluated the combined use of fixed and mobile nodes to establish an urban opportunistic network aimed at the distribution of general interest data. We showed that, as expected, mobility is very crucial for the performance of opportunistic networks. It is not the hindering and challenging property of these networks, but their main enabler. We found that node density does not have to be very high, but that a higher density significantly improves the performance. We observed that the number of contacts is critical, a point that will require anyway further investigation. Combining these findings, we can summarize that, to increase the performance in opportunistic networks by increasing the node density, should be obtained by adding mobile nodes - not fixed ones. Mobile nodes are able to boost the performance, while fixed nodes only increase the overhead. Thus, when needed, adding mobile nodes like for example on buses, trains or taxis, is the best strategy to efficiently establish an urban opportunistic network.

- Increasing the duration of contact time increases the performance of message diffusion. Concretely, we determined that when nodes wait even an extra two seconds each time they contact, this improves the delivery performance while not having a relevant impact on the buffer occupancy or on the protocol overhead. As a proof-of-concept, we showed that Friendly-Sharing can be easily introduced in messaging applications and can effectively improve the collaboration, increasing the delivery ratio and reducing the latency of 
message delivery with a limited impact on buffer utilization. This, combined with a good strategy of buffer management, increases the delivery ratio and reduces the latency of message delivery with a limited impact on buffer utilization. 


\subsection{Publications}

\subsubsection{Journals}

- Herrera-Tapia, Jorge; Hernández-Orallo, Enrique; Tomás, Andrés; Manzoni, Pietro; Calafate, Carlos Tavares; and Cano, Juan-Carlos. "Friendly-Sharing: Improving the Performance of City Sensoring through Contact-Based Messaging Applications". In: Sensors MDPI, September-2016, No. 9:1523, DOI:10.3390/ s16091523. ISSN 1424-8220. Impact Factor: 2.033 (JCR Q1).

An effective way to increase the number of messages that can be shared is to increase the contact duration. We thus introduce the Friendly-Sharing diffusion approach, where, during a contact, the users are aware of the time needed to interchange the messages stored in their buffers, and they can thus decide to wait more time in order to increase the message sharing probability. The performance of this approach is anyway closely related to the size of the buffer in the device. We therefore compare various policies either for the message selection at forwarding times and for message dropping when the buffer is full. We evaluate our proposal with a modified version of the Opportunistic Networking Environment (ONE) simulator and using real human mobility traces.

- Hernández-Orallo, Enrique; Herrera-Tapia, Jorge; Manzoni, Pietro; Cano, Juan-Carlos; and Calafate, Carlos Tavares; Cano, Juan-Carlos; and Manzoni, Pietro. "Evaluating the Impact of Data Transfer Time in ContactBased Messaging Applications". In: IEEE Communications Letters, October2015, Vol. 19, No. 10, pp. 1814-1817. DOI: 10.1109/LCOMM.2015.2472407. ISSN: 1089-7798. Impact Factor: 1.291 (JCR Q2).

In this paper we propose an analytical model based on Delay Differential Equations (DDEs) to evaluate the diffusion of messages in groups taking into account the transmission time of the messages. This model was validated through simulation studies using the ONE simulator. Evaluation results show that considering the impact of data transfer time is of utmost importance, as when message size increases (for example when transmitting short videos), the diffusion is bounded by this transmission time, and the result is that the diffusion time increases slightly when the number of nodes increases (as opposed to the always decreasing diffusion time of the epidemic diffusion with no delay). 


\subsubsection{International Conferences}

- Herrera-Tapia, Jorge; Hernández-Orallo, Enrique; Tomás, Andrés; Calafate, Carlos Tavares; Cano, Juan-Carlos, Zennaro, Marco; and Manzoni, Pietro. "Evaluating the use of sub-gigahertz wireless technologies to improve message delivery in opportunistic networks". In: 2017 14th IEEE International Conference on Networking, Sensing and Control (ICNSC). ISBN: 978-1-46739975-3. May-2017, Calabria-Italy, Core C.

In this paper, we evaluate the use of a sub-gigahertz wireless technology, namely LoRa (Long Range), to establish links between the mobile users in an opportunistic network in order to augment the number of contacts and their duration. We evaluate the performance of LoRa, comparing it with WiFi, using the Epidemic protocol for message diffusion with realistic vehicular traces. Through simulations, we compare the message delivery probability and the network overhead.

- Herrera-Tapia, Jorge; Hernández-Orallo, Enrique; Tomás, Andrés; Manzoni, Pietro; Calafate, Carlos Tavares; and Cano, Juan-Carlos. "Selecting the Optimal Buffer Management for Opportunistic Networks both in Pedestrian and Vehicular Contexts". In: 14th IEEE Annual Consumer Communications \& Networking Conference (CCNC 2017). ISBN: 978-1-5090-6195-2, ISSN: 2331-9860, pp 395-400. January-2017, Las Vegas-USA, Core B.

In this paper we focus on this issue, evaluating the impact of different buffer management approaches in two different scenarios: pedestrian and vehicular. The results show that the best buffer management is the combination of smallest message forwarding and largest message dropping. We also show that a TTL (Time to Live) of 24 hours allows the best diffusion because it corresponds to the daily movement pattern of the nodes.

- Herrera-Tapia, Jorge; Hernández-Orallo, Enrique; Manzoni, Pietro; Tomás, Andrés; Calafate, Carlos Tavares; and Cano, Juan-Carlos. "Evaluating the Impact of Data Transfer Time and Mobility Patterns in Opportunistic Networks". In: 2016 Intl IEEE Conferences on Ubiquitous Intelligence \& Computing, Advanced and Trusted Computing, Scalable Computing and Communications, Cloud and Big Data Computing, Internet of People, and Smart World Congress (UIC/ATC/ScalCom/CBDCom/IoP/SmartWorld). ISBN: 978-1-5090-2771-2. Pp 25-32. DOI: 10.1109/UIC-ATC-ScalCom-CBDComIoP-SmartWorld.2016.0027. July-2016, Toulouse-France, Core B.

In this paper we evaluate the impact of message transmission times in epidemic diffusion processes. We demonstrate how, when certain conditions hold, forcing devices to stop moving to complete the data delivery process can improve their performance. We implemented this mobility model, called 
Forced Stop, in the ONE (Opportunistic Networking Environment) simulator, and we show that, for large message sizes, the diffusion performance is increased.

- Herrera-Tapia, Jorge; Hernández-Orallo, Enrique; Tomás, Andrés; Manzoni, Pietro; Calafate, Carlos Tavares; and Cano, Juan-Carlos. "Improving Message Delivery Performance in Opportunistic Networks Using a Forced-Stop Diffusion Scheme". In: Ad-hoc, Mobile, and Wireless Networks, ADHOCNOW 2016, LNCS 9724, pp. 156-168. ISBN: 9783319405087, DOI: 10.1007/ 978-3-319-40509-4, July-2016, Lille-France, Core B.

In this paper we propose a new diffusion method, called Forced-Stop, that is based on controlling node mobility to guarantee a complete mes- sage transfer. Using the ONE simulator and realistic mobility traces, we compared our proposal with the classical Epidemic diffusion. We show that Forced-Stop improves the message delivery performance, increasing the delivery ratio up to $30 \%$, and reducing the latency of message delivery up to $40 \%$, with a limited impact on buffer utilization and message relaying.

- Herrera-Tapia, Jorge; Manzoni, Pietro; Hernández-Orallo, Enrique; Calafate, Carlos Tavares; and Cano, Juan-Carlos. "Power Consumption Evaluation in Vehicular Opportunistic Networks". In: 2015 IEEE 12th Consumer Communications and Networking Conference (CCNC): CCNC 2015 Workshops - VENITS, pp. 925-930, ISBN: 9781479963904, DOI: 10.1109/CCNC.2015.7158100. January-2015, Las Vegas-USA, Core B.

The goal of this paper is to study the power consumption associated to an opportunistic communication application. This will be achieved by analyzing the different operation stages of an application running on mobile and vehicular devices, using basic statistical analysis calculations on data collected by a logging application. Furthermore, this will allow analysing the impact of this kind of applications regarding battery performance, providing useful information to users and developers.

- Hernández-Orallo, Enrique; Fernández-Delegido, David; Tomás, Andrés; Herrera-Tapia, Jorge; Cano, Juan-Carlos; Calafate, Carlos Tavares; and Manzoni, Pietro. "GRChat: A Contact-based Messaging Application for the Evaluation of Information Diffusion". In: INFOCOMP 2016 The Sixth International Conference on Advanced Communications and Computation (contains MODOPT 2016). ISBN: 978-1-61208-478-7, pp. 32-33. May-2017, Valencia-Spain.

Contact-based messaging applications establish a short-range communication directly between mobile devices, storing the messages in the devices in order to achieve a full dissemination of such messages. When a contact occurs, the mobile devices interchange their stored messages, following a 
epidemic diffusion. No messages are sent or stored in servers. In order to evaluate the diffusion of messages among mobile devices based on opportunistic contacts, we developed GRChat, an Android application that uses Bluetooth as near-by communication protocol. We present some results about the efficiency of peer-to-peer message diffusion depending on message size and devices distance. 


\subsubsection{National Conferences}

- Herrera-Tapia, Jorge; Tomás, Andrés; Hernández-Orallo, Enrique; Manzoni, Pietro; Calafate, Carlos Tavares; and Cano, Juan-Carlos. "Evaluación del Rendimiento de la Difusión de Mensajes Utilizando un Método de Parada Forzada". In: XXVII Jornadas de Paralelismo - SARTECO 2016, pp. 379384, ISBN: 978-84-9012-626-4, September-2016, Salamanca-Spain.

- Herrera-Tapia, Jorge; Hernández-Orallo, Enrique; Manzoni, Pietro; Calafate, Carlos Tavares; Cano, Juan-Carlos; and Alvear, Oscar. "Difusión de mensajes en redes oportunísticas en espacios con alta concentración de personas". In: XXVI Jornadas de Paralelismo - SARTECO 2015, pp. 127-132, ISBN: 978-84-16017-52-2, September-2015, Córdoba-Spain.

- Alvear, Oscar; Calafate, Carlos Tavares; Cano, Juan-Carlos; Manzoni, Pietro; Hernández-Orallo, Enrique; Herrera-Tapia, Jorge. "Metodología para la Monitorización de Ozono en Valencia Mediante Sensores de Gama Baja". In: VI Jornadas de Computación Empotrada (JCE 2015), pp. 57-63, ISBN: 978-84-16017-54-6, September 2015, Córdoba-Spain.

- Herrera-Tapia, Jorge; Hernández-Orallo, Enrique; Calafate, Carlos Tavares; Cano, Juan-Carlos and Manzoni, Pietro. "Evaluación del tiempo de transmisión entre contactos en una red oportunística". In: XXV Jornadas de Paralelismo - SARTECO 2014, pp. 441-446, ISBN: 978-84-697-0329-8, September 2014, Valladolid-Spain 


\subsection{Internship}

During the period of September 2016 to December 2016, I did an internship in the Research Group Sustainable Communication Networks, of the Institute for Telecommunications and High-Frequency Techniques, Faculty of Physics and Electrical Engineering of University of Bremen - Germany. This scholarship was partially funded by the Erasmus program.

\subsection{Projects}

I have participated in the following projects during this thesis period.

- Walkie-Talkie: Vehicular Communication Systems to Enable Safer, Smarter, and Greener Transportation", which was funded by the Ministerio de Economía y Competitividad, Spain, under Grant TIN2011-27543-C03-01.

- SmartCarPhone: Toward Seamless Smartphone and Vehicle Integration to Connect Drivers with Sensors and the Environment in a Holistic ServiceOriented Architecture" which was funded by Ministerio de Economía y Competitividad, Programa Estatal de Investigación, Desarrollo e Innovación Orientada a los Retos de la Sociedad, Proyectos I+D+I 2014, Spain, under Grant TEC2014-52690-R.

- Analysis of the mobility and information persistence in Vehicular Networks. Aplication in accidents management. Ministerio de Economía y Competitividad, Programa Estatal de Investigación, Desarrollo e Innovación Orientada a los Retos de la Sociedad, Proyectos I+D+I 2014, Spain, under Grant TEC2014-52690-R, the Generalitat Valenciana, Spain, under Grant $\mathrm{AICO} / 2015 / 108$. 


\subsection{Future Work}

In the field of opportunistic wireless networks there are many subjects that can be improved. In this thesis, we have investigated about this kind of networks, according to the acquired expertise in this process. Thus, we propose some of them to be considered in future works.

- The results obtained in this research work can be a relevant indication to the designers of opportunistic network applications that could integrate in their products strategies to inform the user about the need to temporarily stop in order to increase the overall data delivery. Our interest in this type of analysis, on the long term, is focused on the design of cross-layer content distribution strategy to improve information sharing in opportunistic networks, and to provide a clear insight on how to develop and deploy efficient cooperative applications.

- Encourage apps developers to use buffer management policies in their products, moreover, combined with strategies to inform the user about the need to temporarily stop in order to encourage the collaboration level between mobile users.

- On the long term, our interest in this analysis is to increase the battery life by optimizing applications without losing their functionalities. Future works are focused on the following aspects: a) exploiting sensor information in the design and implementation of energy-efficient applications, and b) encouraging the collaboration between mobile devices by using of mobile apps designed with energy efficiency constraints.

- Improve GRChat app with more functionalities in order to extend this application to more users.

- About other type of data transmission technologies, the next step in our research will be to perform experiments with a real prototype implementation using embedded devices with LoRa data transmission to validate our simulation setup.

- Design and implement in The ONE simulator functionalities of lower layers, in order to get more information about the simulations. 


\section{Appendix A}

\section{Acronyms}

\begin{tabular}{ll} 
AODV & Ad hoc On-Demand Distance Vector \\
AP & Access Point \\
API & Application Programming Interface \\
BLE & Bluetooth Low Energy \\
BP & Bundel Protocol \\
CDF & Cumulative Distribution Function \\
DAER & Distance Aware Epidemic Routing \\
DC & Direct Current \\
D2D & Device to Device \\
DDE & Delay Differential Equations \\
DES & Discret Event Simulator \\
DSR & Dynamic Source Routing \\
DTN & Delay Tolerant Network \\
DTNRG & Delay Tolerant Network Research Group \\
DV-CAST & Distributed Vehicular BroadCast \\
\hline
\end{tabular}


ETSI European Telecommunications Standards Institute

FCC Federal Communications Commission

FEC Forward Error Correction

FSPL Free Space Path Loss

FIFO First In First Out

GIS Geographic Information Service

GPS Global Positioning System

GUI Graphical User Interface

HTTP Hypertext Transfer Protocol

IETF Internet Engineering Task Force

IoT Internet of Things

IPN InterPlanetary Network

IP Internet Protocol

ITS Intelligent Transport System

IVC Inter-Vehicle Communication

LAN Local Area Network

LOS Line of Sight

LoRa Long Range

LPWAN Low Power Wireless Area Personal Networks

6LoWPAN IPv6 over Low power Wireless Personal Area Networks

LTE Long Term Evolution

MAC Media Access Control

M2M Machine to Machine

MANET Mobile Ad-hoc NETwork

MB Mega Bytes

Mb Mega bits 


\begin{tabular}{ll} 
MTU & Maximum Transfer Unit \\
MIT & Massachusetts Institute of Technology \\
NS & Navigation System \\
ns-3 & Network Simulator 3 \\
NS-2 & Network Simulator 2 \\
NCCU & National Chengchi University \\
OLSR & Optimized Link State Routing \\
ONE & Opportunistic Network Environment \\
OSI & Open Systems Interconnection \\
OppNets & Opportunistic Networks \\
OPS & Opportunistic Protocol Simulator \\
PAN & Personal Area Network \\
P2P & Peer-to-Peer \\
PDU & Protocol Data Unit \\
PER & Packet Error Rate \\
PRR & Packet Reception Ratios \\
RSU & Road Side Unit \\
RTT & Round Trip Time \\
RWP & Random Way Point \\
RW & Random Walk \\
SFO & San Francisco \\
SLAW & Self-similar Least Action \\
SOLAR & Sociological Orbit model \\
SUMO & Simulation of Urban MObility \\
SWIM & Small World In Motion \\
TCP & Transmission Control Protocol \\
\hline PW
\end{tabular}


TLW Truncated Levy Walk

TTL Time To Live

TVC Time Variant Community model

UDG Unit Disk Graph

VANET Vehicular Ad-Hoc Network

VDTN Vehicular Delay Tolerant Network

VN Vehicular Network

WAN Wide Area Network

WAVE Wireless Access for Vehicular Environment

WiFi Wireless Fidelity

WDM Work Day Movement 


\section{Bibliography}

[1] A technical overview of LoRa and LoRaWAN (LoRa Alliance). https : / / ww . lora-alliance . org / portals / 0 / documents / whitepapers / LoRaWAN101.pdf. Accessed: 3/11/2016 (cited on p. 15).

[2] U. Aalto. Liberouter. http://www. ict-scampi . eu/results/scampiliberouter/. Accessed: 14-12-2016 (cited on p. 34).

[3] M. Z. Abhinav Pathak Y Charlie Hu. "Fine Grained Energy Accounting on Smartphones with Eprof." In: Proceedings of the ACM SIGOPS EuroSys 2012 Conference (2012) (2012), pp. 29-42 (cited on p. 32).

[4] C. S. de Abreu and R. M. Salles. "Modeling message diffusion in epidemical DTN." In: Ad Hoc Networks 16 (Benidorm, Spain, 2014), pp. 197-209 (cited on p. 85).

[5] D.-g. Akestoridis, N. Papanikos, and E. Papapetrou. "Exploiting Social Preferences for Congestion Control in Opportunistic Networks." In: Section V (2014), pp. 413-418 (cited on p. 26).

[6] A. Al Hanbali, M. Ibrahim, V. Simon, E. Varga, and I. Carreras. "A Survey of Message Diffusion Protocols in Mobile Ad Hoc Networks." In: Proceedings of the 3rd International Conference on Performance Evaluation Methodologies and Tools (2008), 82:1-82:16 (cited on p. 6).

[7] S. Al-Sultan, M. M. Al-Doori, A. H. Al-Bayatti, and H. Zedan. "A comprehensive survey on vehicular Ad Hoc network." In: Journal of Network and Computer Applications 37.1 (2014), pp. 380-392 (cited on p. 58). 
[8] M. J. Alenazi, Y. Cheng, D. Zhang, and J. P. Sterbenz. "Epidemic routing protocol implementation in ns-3." In: Proceedings of the 2015 Workshop on ns-3. ACM. 2015, pp. 83-90 (cited on p. 25).

[9] R. Amici, M. Bonola, L. Bracciale, A. Rabuffi, P. Loreti, and G. Bianchi. "Performance Assessment of an Epidemic Protocol in VANET Using Real Traces." In: Procedia Computer Science 40 (2014), pp. 92-99 (cited on pp. $44,45,58)$.

[10] M. Avvenuti, P. Corsini, P. Masci, and A. Vecchio. "Opportunistic computing for wireless sensor networks." In: Mobile Adhoc and Sensor Systems. MASS 200\%. IEEE Internatonal Conference on (2007), pp. 1-6 (cited on p. 32$)$.

[11] F. Bai and A. Helmy. "A SURVEY OF MOBILITY MODELS." In: (2004) (cited on p. 11).

[12] N. Banerjee, M. D. Corner, D. Towsley, and B. N. Levine. "Relays, Base Stations, and Meshes: Enhancing Mobile Networks with Infrastructure." In: Proceedings of the 14th ACM International Conference on Mobile Computing and Networking. MobiCom '08. New York, NY, USA: ACM, 2008, pp. 81-91 (cited on p. 8).

[13] C. Bettstetter and C. Wagner. "The Spatial Node Distribution of the Random Waypoint Mobility Model." In: Proc. 1st German Workshop Mobile Ad-Hoc Network. WMAN (2002), pp. 41-58 (cited on p. 88).

[14] J. Bischoff, M. Maciejewski, and A. Sohr. "Analysis of Berlin's taxi services by exploring GPS traces." In: 2015 International Conference on Models and Technologies for Intelligent Transportation Systems, MT-ITS 2015 December 2012 (2015), pp. 209-215 (cited on p. 58).

[15] Bluetooth, SIG. "Bluetooth specification version 5.0." In: Bluetooth SIG (2014) (cited on p. 14).

[16] C. Boldrini, M. Conti, and A. Passarella. "Modelling Data Dissemination in Opportunistic Networks." In: Proceedings of the third ACM workshop on Challenged networks - CHANTS '08 (San Francisco, USA, 2008), pp. 8996 (cited on pp. 68, 84, 100).

[17] C. Boldrini and A. Passarella. "HCMM: Modelling Spatial and Temporal Properties of Human Mobility Driven by Users' Social Relationships." In: 
Comput. Commun. 33.9 (June 2010), pp. 1056-1074. ISSN: 0140-3664. DOI: 10.1016/j.comcom.2010.01.013 (cited on p. 13).

[18] L. Bracciale, M. Bonola, P. Loreti, G. Bianchi, R. Amici, and A. Rabuffi. "CRAWDAD dataset roma/taxi (v. 2014-07-17)." In: (2014) (cited on pp. 58, $61,68)$.

[19] J. Burgess, B. Gallagher, D. Jensen, and B. N. Levine. "MaxProp: Routing for vehicle-based disruption-tolerant networks." In: Proceedings - IEEE INFOCOM 00.c (2006). ISSN: 0743166X. DOI: 10.1109/INFOCOM . 2006. 228 (cited on pp. 6, 19, 22).

[20] S. Burleigh, E. Jennings, and J. Schollcraft. "Autonomous Congestion Control in Delay-Tolerant Networks." In: SpaceOps (2006), pp. 1-10 (cited on p. 26).

[21] L Cai, J. P. Pan, X. M. Shen, and J. W. Mark. "Peer collaboration in wireless ad hoc networks." In: Networking 2005: Networking Technologies, Services, and Protocols; Performance of Computer and Communication Networks; Mobile and Wireless Communications Systems 3462 (2005), pp. 840852 (cited on p. 100).

[22] T. Camp, J. Boleng, and V. Davies. "A survey of mobility models for ad hoc network research." In: vol. 2. 5. Wiley Online Library, 2002, pp. 483502 (cited on p. 11).

[23] G. Cardone, A. Cirri, A. Corradi, L. Foschini, R. Ianniello, and R. Montanari. "Crowdsensing in Urban areas for city-scale mass gathering management: Geofencing and activity recognition." In: IEEE Sensors Journal 14.12 (2014), pp. 4185-4195. ISSN: 1530437X. DOI: 10.1109/JSEN . 2014. 2344023 (cited on p. 100).

[24] G. Cardone, L. Foschini, P. Bellavista, A. Corradi, C. Borcea, M. Talasila, and R. Curtmola. "Fostering participaction in smart cities: A geo-social crowdsensing platform." In: IEEE Communications Magazine 51.6 (2013), pp. 112-119. ISSN: 01636804. DOI: 10.1109/MCOM.2013.6525603 (cited on p. 100).

[25] N. Chakchouk. "A Survey on Opportunistic Routing in Wireless Communication Networks." In: IEEE Communications Surveys Tutorials 17.4 (2015), pp. 2214-2241. ISSN: 1553-877X. DOI: 10 . 1109 / COMST . 2015 . 2411335 (cited on pp. 6, 17). 
[26] C. Chen and Y. Zhu. "An evaluation of vehicular networks with real traces." In: Proceedings of the International Conference on Parallel and Distributed Systems - ICPADS (2012), pp. 716-717 (cited on p. 44).

[27] Q. Chen. "Multi-Metric Opportunistic Routing for VANETs in Urban Scenario." In: 2011 International Conference on Cyber-Enabled Distributed Computing and Knowledge Discovery (2011), pp. 118-122 (cited on p. 58).

[28] X. Chen, C. Shang, B. Wong, W. Li, and S. Oh. "Efficient Multicast Algorithms in Opportunistic Mobile Social Networks using Community and Social Features." In: Computer Networks 111 (2016), pp. 71-81. ISSN: 13891286. DOI: 10.1016/j.comnet.2016.07.007 (cited on p. 100).

[29] M. Ciocan, C. Dobre, V. Cristea, X. C. Mavromoustakis, and G. Mastorakis. "Analysis of Vehicular Storage and Dissemination Services Based on Floating Content." In: Mobile Networks and Management: 6th International Conference, MONAMI 2014, Würzburg, Germany, September 22-26, 2014, Revised Selected Papers. Cham: Springer International Publishing, 2015, pp. 387-400 (cited on p. 45).

[30] S. Ciraci, J. Daily, J. Fuller, A. Fisher, L. Marinovici, and K. Agarwal. "FNCS: A Framework for Power System and Communication Networks Co-simulation." In: Proceedings of the Symposium on Theory of Modeling \& Simulation - DEVS Integrative. DEVS '14. Tampa, Florida: Society for Computer Simulation International, 2014, 36:1-36:8 (cited on p. 24).

[31] P. Costa, D. Gavidia, B. Koldehofe, H. Miranda, M. Musolesi, and O. Riva. "When cars start gossiping." In: Proceedings of the 6th workshop on Middleware for network eccentric and mobile applications - MiNEMA '08 (2008), pp. 1-4 (cited on p. 68).

[32] E. M. Daly and M. Haahr. "Social network analysis for routing in disconnected delay-tolerant MANETs." In: MobiHoc 'O7 (2007), pp. 32-40 (cited on p. 26).

[33] C. S. De Abreu and R. M. Salles. "Modeling Message Diffusion in Epidemical DTN." In: Ad Hoc Networks 16 (2014), pp. 197-209 (cited on p. 8).

[34] J. A. Dias, J. N. Isento, V. N.G. J. Soares, F. Farahmand, and J. J.P. C. Rodrigues. "Testbed-based Performance Evaluation of Routing Protocols for Vehicular Delay-Tolerant Networks." In: IEEE, 45th Annual Simulation Symposium (2011), pp. 51-55 (cited on p. 6). 
[35] J. A. Dias, J. J. Rodrigues, and L. Zhou. "Cooperation advances on vehicular communications: A survey." In: Vehicular Communications 1.1 (2014), pp. $22-32$ (cited on pp. 58, 69).

[36] L. Dong. "Opportunistic media access control and routing for delay-tolerant mobile ad hoc networks." In: Wireless Networks 18 (2012), pp. 949-965 (cited on p. 6).

[37] N. Eagle and A. Pentland. "Social serendipity: mobilizing social software." In: Pervasive Computing, IEEE 4.2 (2005), pp. 28-34 (cited on p. 12).

[38] F. Ekman, A. Keränen, J. Karvo, and J. Ott. "Working Day Movement Model." In: Proceedings of the 1st ACM SIGMOBILE Workshop on Mobility Models. MobilityModels '08. Hong Kong, Hong Kong, China: ACM, 2008, pp. 33-40. ISBN: 978-1-60558-111-8 (cited on p. 13).

[39] V. Erramilli, A. Chaintreau, M. Crovella, and C. Diot. "Delegation forwarding." In: Proceedings of the 9th ACM international symposium on Mobile ad hoc networking and computing - MobiHoc '08 (2008), pp. 251-259 (cited on p. 26).

[40] V. Erramilli and M. Crovella. "Forwarding in opportunistic networks with resource constraints." In: Proceedings of the third ACM workshop on Challenged networks - CHANTS '08 (San Francisco, USA, 2008), pp. 41-47 (cited on pp. 45, 69, 100).

[41] K. Fall. "A delay-tolerant network architecture for challenged internets." In: Proceedings of the 2003 conference on Applications, technologies, architectures, and protocols for computer communications - SIGCOMM '03 (2003), p. 27 (cited on p. 21).

[42] G Fathima and R. Wahidabanu. "Buffer management for preferential delivery in opportunistic delay tolerant networks." In: International Journal of Wireless and Mobile Networks (IJWMN) 3 (2011), pp. 15-28 (cited on pp. 45, 100).

[43] Z. Feng and K.-W. Chin. "A Unified Study of Epidemic Routing Protocols and their Enhancements." In: Parallel and Distributed Processing Symposium Workshops PhD Forum (IPDPSW), 2012 IEEE 26th International (Shanghai, China, 2012), pp. 1484-1493 (cited on p. 85). 
[44] S. Ferretti. "Shaping opportunistic networks." In: Computer Communications 36 (2013), pp. 481-503 (cited on pp. 5, 58).

[45] A. Förster, A. Udugama, C. Görg, K. Kuladinithi, A. Timm-Giel, and A. Cama-Pinto. "A Novel Data Dissemination Model for Organic Data Flows." In: 7th EAI International Conference on Mobile Networks and Management (MONAMI), Santander, Spain. 2015 (cited on p. 70).

[46] A. Förster, K. Garg, H. A. Nguyen, and S. Giordano. "On Context Awareness and Social Distance in Human Mobility Traces." In: Third ACM International Workshop on Mobile Opportunistic Networks. MobiOpp '12 (Zürich, Switzerland, 2012), pp. 5-12 (cited on pp. 68, 100).

[47] T. M. J. Fruchterman and E. M. Reingold. "Force-Directed." In: SoftwarePractice And Experience Noviembre, 21 (1991), pp. 1129-1164 (cited on p. 115).

[48] Q. Fu, L. Zhang, W. Feng, and Y. Zheng. "DAWN: A density adaptive routing algorithm for vehicular delay tolerant sensor networks." In: 2011 49th Annual Allerton Conference on Communication, Control, and Computing, Allerton 2011 (2011), pp. 1250-1257 (cited on p. 58).

[49] S. Gaito, E. Pagani, and G. Rossi. "Fine-Grained Tracking of Human Mobility in Dense Scenarios." In: Proceedings of the 6th Annual IEEE Communications Society Conference on Sensor, Mesh and Ad Hoc Communications and Networks Workshops, 2009. SECON Workshops '09. 2009, pp. 13 (cited on p. 12).

[50] J. Ghosh, S. J. Philip, and C. Qiao. "Sociological orbit aware location approximation and routing (SOLAR) in $\{$ MANET\}." In: Ad Hoc Networks 5.2 (2007), pp. 189 -209. ISSN: 1570-8705 (cited on p. 13).

[51] Google Android Copresence. https : / / developer . chrome . com / apps / copresence. Accessed: 4/10/2015 (cited on p. 86).

[52] R. Groenevelt, P. Nain, and G. Koole. "The message delay in mobile ad hoc networks." In: Performance Evaluation 62 (2005), pp. 210-228 (cited on pp. 8,9).

[53] Z. J. Haas and T. Small. "A New Networking Model for Biological Applications of Ad Hoc Sensor Networks." In: IEEE/ACM Transactions on Networking 14.1 (Feb. 2006), pp. 27-40 (cited on p. 8). 
[54] O. R. Helgason and K. V. Jónsson. "Opportunistic networking in OMNeT++." In: Proceedings of the 1st international conference on Simulation tools and techniques for communications, networks and systems and workshops, Simutools 2008, Marseille, France, March 03 - 07, 2008. 2008 (cited on p. 23).

[55] O. R. Helgason and S. T. Kouyoumdjieva. "Enabling multiple controllable radios in OMNeT++ nodes." In: Proceedings of the 4th International ICST Conference on Simulation Tools and Techniques, SIMUTools 2011, Barcelona, Spain, March 21 - 25, 2011. 2011 (cited on p. 23).

[56] E. Hernandez-Orallo, M. Serrat Olmos, J.-C. Cano, C. Calafate, and P. Manzoni. "CoCoWa: A Collaborative Contact-Based Watchdog for Detecting Selfish Nodes." In: Mobile Computing, IEEE Transactions on 14.6 (2015), pp. 1162-1175 (cited on p. 8).

[57] E. Hernández-Orallo, J. C. Cano, C. T. Calafate, and P. Manzoni. "New approaches for characterizing inter-contact times in opportunistic networks." In: Ad Hoc Networks 52 (2016). Modeling and Performance Evaluation of Wireless Ad Hoc Networks, pp. $160-172$ (cited on p. 12).

[58] E. Hernández-Orallo, J. C. Cano, C. T. Calafate, and P. Manzoni. "New approaches for characterizing inter-contact times in opportunistic networks." In: Ad Hoc Networks 52 (2016), pp. 160-172 (cited on pp. 44, 49, 61).

[59] E. Hernández-orallo, D. Fernández-Delegido, J. Herrera-tapia, J.-c. Cano, C. T. Calafate, and P. Manzoni. "GRChat : A Contact-based Messaging Application for the Evaluation of Information Diffusion." In: In Proceedings of the Sixth International Conference on Advanced Communications and Computation, INFOCOMP 2016 c (2016), pp. 32-33 (cited on p. 112).

[60] E. Hernández-orallo, J. Herrera-tapia, J.-c. Cano, C. T. Calafate, and P. Manzoni. "Evaluating the Impact of Data Transfer Time in Contact-Based Messaging Applications." In: IEEE Communications Letters 19 (2015), pp. 1814-1817 (cited on p. 85).

[61] E. Hernández-Orallo, M. Murillo-Arcila, C. T. Calafate, J. C. Cano, J. A. Conejero, and P. Manzoni. "Analytical evaluation of the performance of contact-Based messaging applications." In: Computer Networks 111 (2016), pp. $45-54$ (cited on p. 8). 
[62] J. Herrera-Tapia, E. Hernández-Orallo, A. Tomás, P. Manzoni, C. Tavares Calafate, and J.-C. Cano. "Friendly-Sharing: Improving the Performance of City Sensoring through Contact-Based Messaging Applications." In: Sensors 16.9 (2016), p. 1523. ISSN: 1424-8220 (cited on p. 61).

[63] J. Herrera-tapia, P. Manzoni, E. Hern, C. T. Calafate, and J.-c. Cano. "Power Consumption Evaluation in Vehicular Opportunistic Networks." In: IEEE 12th CCNC 2015 Workshops - VENITS (2015), pp. 925-930 (cited on p. 85).

[64] W. j. Hsu, T. Spyropoulos, K. Psounis, and A. Helmy. "Modeling TimeVariant User Mobility in Wireless Mobile Networks." In: IEEE INFOCOM 2007 - 26th IEEE International Conference on Computer Communications. 2007, pp. 758-766 (cited on p. 13).

[65] http://www.raspberrypi.org/products. Model B - Raspberry Pi (cited on p. 34$)$.

[66] W. Hu, J. Xie, and Z. Zhang. "Practical Opportunistic Routing in HighSpeed Multi-Rate Wireless Mesh Networks Categories and Subject Descriptors." In: (2013), pp. 127-136 (cited on p. 58).

[67] C.-M. Huang, K.-c. Lan, and C.-Z. Tsai. "A Survey of Opportunistic Networks." In: 22nd International Conference on Advanced Information Networking and Applications (2008), pp. 1672-1677 (cited on p. 6).

[68] P. Hui, A. Chaintreau, J. Scott, R. Gass, J. Crowcroft, and C. Diot. "Pocket switched networks and human mobility in conference environments." In: Proceedings of the 2005 ACM SIGCOMM workshop on Delay-tolerant networking. WDTN '05. New York, NY, USA: ACM, 2005, pp. 244-251 (cited on p. 12).

[69] P. Hui, J. Crowcroft, and E. Yoneki. "BUBBLE Rap: Social-based forwarding in delay-tolerant networks." In: IEEE Transactions on Mobile Computing 10.11 (2011), pp. 1576-1589 (cited on pp. 19, 26).

[70] IEEE. "IEEE Standard for Information technology." In: IEEE Std 802.112012 (2012) (cited on p. 13).

[71] IEEE 802.11 Wireless Local Area Networks - The Working Group for WLAN Standards. http : //www . ieee802 . org/11/. Accessed: 2017-0220 (cited on p. 13). 
[72] "IEEE Standard for Local and metropolitan area networks-Part 15.4: LowRate Wireless Personal Area Networks (LR-WPANs)." In: IEEE Std 802.15.42011 (Revision of IEEE Std 802.15.4-2006) (2011), pp. 1-314 (cited on p. 14).

[73] Internet of Things. http://www . internet-of-things-research . eu/ index.html/. Accessed: 03/11/2016 (cited on p. 14).

[74] W. D. Ivancic. "Security analysis of DTN architecture and bundle protocol specification for space-based networks." In: IEEE Aerospace Conference Proceedings (2010) (cited on p. 21).

[75] S Jain, K Fall, and R Patra. "Routing in a Delay Tolerant Network." In: SIGCOMM '04: Proceedings of the 2004 conference on Applications, technologies, architectures, and protocols for computer communications (2004), pp. 145-158 (cited on pp. 17, 22).

[76] A. Jindal and K. Psounis. "Performance Analysis of Epidemic Routing under Contention." In: Proceedings of the 2006 International Conference on Wireless Communications and Mobile Computing (2006), pp. 539-544 (cited on p. 85).

[77] V Kanakaris, D Ndzi, and D Azzi. "Ad-hoc Networks Energy Consumption : A review of the Ad-Hoc Routing Protocols." In: 3 (2010), pp. 162-167 (cited on p. 32).

[78] J. Kangasharju, J. Ott, and O. Karkulahti. "Floating content: Information availability in urban environments." In: 8th IEEE Conf. on Pervasive Computing and Communications Workshops. 2010, pp. 804-808 (cited on p. 2).

[79] J. Kangasharju, J Ott, and O Karkulahti. "Floating Content: Information Availability in Urban Environments." In: Proceedings of the 8th Annual IEEE (2010), pp. 804-808 (cited on p. 32).

[80] M. Karaliopoulos. "Assessing the vulnerability of DTN data relaying schemes to node selfishness." In: Communications Letters, IEEE 13.12 (2009), pp. 923 -925 (cited on p. 8).

[81] T. Karkkainen and J. Ott. "Liberouter: Towards autonomous neighborhood networking." In: 2014 11th Annual Conference on Wireless On-demand 
Network Systems and Services (WONS) (2014), pp. 162-169 (cited on p. 34).

[82] C. F. F. Karney. "Transverse Mercator with an accuracy of a few nanometers." In: Journal of Geodesy 85.8 (2011), pp. 475-485 (cited on pp. 48, $61)$.

[83] R. Karp, C. Schindelhauer, S. Shenker, and B. Vocking. "Randomized rumor spreading." In: Foundations of Computer Science, 2000. Proceedings. 41st Annual Symposium on. IEEE. 2000, pp. 565-574 (cited on pp. 19, 24, $77)$.

[84] A. Keränen, J. Ott, and T. Kärkkäinen. "The ONE Simulator for DTN Protocol Evaluation." In: Proceedings of the 2Nd International Conference on Simulation Tools and Techniques. Simutools '09. Rome, Italy, 2009, 55:1-55:10. ISBN: 978-963-9799-45-5 (cited on pp. 20, 21).

[85] A. Keränen, J. Ott, and T. Kärkkäinen. "The ONE simulator for DTN protocol evaluation." In: Proceedings of the Second International ICST Conference on Simulation Tools and Techniques (Rome, Italy, 2009) (cited on pp. 17, 87, 102).

[86] W. O. Kermack and A. G. McKendrick. "A Contribution to the Mathematical Theory of Epidemics." In: Proceedings of the Royal Society of London A: Mathematical, Physical and Engineering Sciences 115 (1927), pp. 700721 (cited on p. 85).

[87] P. M. Khilar and S. K. Bhoi. "Vehicular communication: a survey." In: IET Networks 3.3 (2014), pp. 204-217 (cited on p. 58).

[88] I Komnios, S Diamantopoulos, and V Tsaoussidis. "Evaluation of dynamic DTN routing protocols in space environment." In: Satellite and Space Communications, 2009. IWSSC 2009. International Workshop on (2009), pp. 191195 (cited on p. 6).

[89] S. T. Kouyoumdjieva, S. Chupisanyarote, O. R. Helgason, and G. Karlsson. "Caching Strategies in Opportunistic Networks." In: Proceedings of the IEEE International Symposium on World of Wireless, Mobile and Multimedia Networks, WoWMoM 2012. 2012 (cited on p. 23). 
[90] Y. Y.I.R.S. C. Kyunghan Lee Joohyun Lee. "Mobile Data Offloading : How Much Can WiFi Deliver ?" In: IEEE/ACM Transactions on Networking 21 (2013), pp. 536-550 (cited on p. 32).

[91] J. Lakkakorpi, M. Pitkänen, and J. Ott. "Using buffer space advertisements to avoid congestion in mobile opportunistic DTNs." In: Lecture Notes in Computer Science (including subseries Lecture Notes in Artificial Intelligence and Lecture Notes in Bioinformatics) 6649 LNCS (2011), pp. 386397 (cited on p. 26).

[92] J.-Y. Le Boudec. Performance Evaluation of Computer and Communication Systems. EPFL Press, CRC Press, 2010 (cited on p. 6).

[93] J. Leguay, A. Lindgren, J. Scott, T. Friedman, and J. Crowcroft. "Opportunistic Content Distribution in an Urban Setting." In: Proceedings of the 2006 SIGCOMM Workshop on Challenged Networks. CHANTS '06. New York, NY, USA: ACM, 2006, pp. 205-212 (cited on p. 12).

[94] I. Leontiadis, P. Costa, and C. Mascolo. "Extending Access Point Connectivity through Opportunistic Routing in Vehicular Networks." In: 2010 Proceedings IEEE INFOCOM (2010), pp. 1-5 (cited on p. 58).

[95] A. Lindgren, A. Doria, and O. Schelén. "Probabilistic Routing in Intermittently Connected Networks." In: (2004). Ed. by P. Dini, P. Lorenz, and J. N. de Souza, pp. 239-254. DOI: 10.1007/978-3-540-27767-5_24 (cited on pp. $6,18,19,22)$.

[96] LoRa Alliance. "Mobile Experts White Paper for LoRa Alliance: Where does LoRa Fit in the Big Picture?" In: (2015) (cited on p. 15).

[97] LoRa Alliance - Wide Area Networks for IoT. https : / / www . Ioraalliance.org/. Accessed: 2017-02-20 (cited on p. 14).

[98] LoRa Device Developer Guide (ORANGE). https : / / partner . orange . com/wp-content/uploads / 2016/04/LoRa-Device-Developer-GuideOrange.pdf. Accessed: 4/10/2016 (cited on p. 14).

[99] P. Luo, H. Huang, W. Shu, M. Li, and M.-Y. Wu. "NET 07-2 - Performance Evaluation of Vehicular DTN Routing under Realistic Mobility Models." In: 2008 IEEE Wireless Communications and Networking Conference (2008), pp. 2206-2211 (cited on pp. 58, 69). 
[100] X. Mao, S. Tang, X. Xu, X.-Y. Li, and H. Ma. "Energy Efficient Opportunistic Routing in Wireless Sensor Networks." In: Parallel and Distributed Systems, IEEE Transactions on (2011), pp. 1934-1942 (cited on p. 32).

[101] J. M. Marquez-Barja, H. Ahmadi, S. M. Tornell, C. T. Calafate, J. C. Cano, P. Manzoni, and L. A. DaSilva. "Breaking the vehicular wireless communications barriers: Vertical handover techniques for heterogeneous networks." In: IEEE Transactions on Vehicular Technology 64.12 (2015), pp. 5878-5890 (cited on p. 58).

[102] K. Massri, A. Vernata, and A. Vitaletti. "Routing protocols for delay tolerant networks: a quantitative evaluation." In: ACM PM2HW2N'12 wireless and wired networks (2012), pp. 107-114 (cited on p. 6).

[103] N. Mehta and M. Shah. "Performance Evaluation of Efficient Routing Protocols in Delay Tolerant Network under Different Human Mobility Models." In: 8 (2015), pp. 169-178 (cited on p. 85).

[104] A. Mei and J. Stefa. "SWIM: A Simple Model to Generate Small Mobile Worlds." In: CoRR abs/0809.2730 (2008) (cited on pp. 12, 13).

[105] W. Moreira and P. Mendes. "Impact of human behavior on social opportunistic forwarding." In: Ad Hoc Networks 25, Part B (2015), pp. 293 -302. ISSN: 1570-8705 (cited on p. 44).

[106] V. F. Mota, F. D. Cunha, D. F. Macedo, J. M. Nogueira, and A. A. Loureiro. "Protocols, mobility models and tools in opportunistic networks: A survey." In: Computer Communications 48 (2014), pp. 5-19. ISSN: 0140-3664 (cited on p. 6).

[107] A. Munjal, T. Camp, and W. Navidi. "SMOOTH: a simple way to model human mobility." In: Proceedings of the 14th International Symposium on Modeling Analysis and Simulation of Wireless and Mobile Systems, MSWiM 2011, Miami, Florida, USA, October 31 - November 4, 2011. 2011, pp. 351-360 (cited on p. 13).

[108] A. Munjal, W. C. Navidi, and T. Camp. "Steady-State of The SLAW Mobility Model." In: JCM 9.4 (2014), pp. 322-331 (cited on p. 13).

[109] E. Natalizio and V. Loscrí. "Controlled Mobility in Mobile Sensor Networks: Advantages, Issues and Challenges." In: Telecommunication Systems 52.4 (2013), pp. 2411-2418 (cited on p. 44). 
[110] V. V. Neena and V. M. A. Rajam. "Performance analysis of epidemic routing protocol for opportunistic networks in different mobility patterns." In: 2013 International Conference on Computer Communication and Informatics (Coimbatore, India, 2013), pp. 1-5 (cited on pp. 44, 85).

[111] S. C. Nelson, M. Bakht, and R. Kravets. "Encounter - Based Routing in DTNs." In: Ieee Infocom'09 (2009), pp. 846-854 (cited on p. 26).

[112] J. Niu, J. Guo, Q. Cai, N. Sadeh, and S. Guo. "Predict and Spread: an Efficient Routing Algorithm for Opportunistic Networking." In: Wireless Communications and Networking Conference (WCNC), 2011 IEEE (Cancún, México, 2011), pp. 498-503 (cited on pp. 5, 18, 58).

[113] "Opportunistic Routing." In: Mobile Ad Hoc Networking. John Wiley \& Sons, Inc., 2013, pp. 419-452. ISBN: 9781118511305

. DOI: $10.1002 / 9781118511305 . \operatorname{ch} 11$ (cited on p. 17).

[114] J. Ott, E. Hyytiä, P. Lassila, J. Kangasharju, and S. Santra. "Floating content for probabilistic information sharing." In: Pervasive and Mobile Computing 7 (2011), pp. 671-689 (cited on p. 32).

[115] J. Ott, E. Hyytia, P. Lassila, T. Vaegs, and J. Kangasharju. "Floating content: Information sharing in urban areas." In: 2011 IEEE International Conference on Pervasive Computing and Communications (PerCom) (2011), pp. 136-146 (cited on p. 34).

[116] L. Pajevic, G. Karlsson, and O. Helgason. "Epidemic Content Distribution: Empirical and Analytic Performance." In: Proceedings of the 16th ACM International Conference on Modeling, Analysis $8 \# 38$; Simulation of Wireless and Mobile Systems, MSWiM '13 (2013), pp. 335-340 (cited on p. 84).

[117] D. Pan, Z. Ruan, N. Zhou, X. Liu, and Z. Song. "A comprehensive-integrated buffer management strategy for opportunistic networks." In: EURASIP Journal on Wireless Communications and Networking (2013), pp. 1-10 (cited on pp. 45, 69, 100).

[118] N. Papanikos, D.-G. Akestoridis, and E. Papapetrou. Adyton: A Network Simulator for Opportunistic Networks. [Online].

Available: https://github.com/npapanik/Adyton. 2015 (cited on p. 25). 
[119] N. Papanikos and E. Papapetrou. "Coordinating replication decisions in multi-copy routing for opportunistic networks." In: International Conference on Wireless and Mobile Computing, Networking and Communications (2014), pp. 8-13 (cited on p. 26).

[120] A. Passarella and M. Conti. "Characterising aggregate inter-contact times in heterogeneous opportunistic networks." In: Proceedings of the 10th international IFIP TC 6 conference on Networking - Volume Part II. NETWORKING'11. Berlin, Heidelberg: Springer-Verlag, 2011, pp. 301-313 (cited on p. 12).

[121] L. Pelusi, A. Passarella, and M. Conti. "Opportunistic Networking: Data Forwarding in Disconnected Mobile Ad Hoc Networks." In: IEEE Communications Magazine (2006), pp. 134-141 (cited on pp. 5, 17).

[122] C. Peng, Q. Zhang, M. Zhao, and Y. Yao. "Opportunistic network-coded cooperation in wireless networks." In: IEEE Wireless Communications and Networking Conference, WCNC (2007), pp. 3360-3365 (cited on p. 100).

[123] S. Pérez-Sánchez, J. M. Cabero, and I. Urteaga. "Wired/Wireless Internet Communications: 13th International Conference, WWIC 2015, Malaga, Spain, May 25-27, 2015, Revised Selected Papers." In: ed. by C. M. AguayoTorres, G. Gómez, and J. Poncela. Cham: Springer International Publishing, 2015. Chap. DTN Routing Optimised by Human Routines: The HURRy Protocol, pp. 299-312. ISBN: 978-3-319-22572-2 (cited on pp. 44, 100).

[124] M. Piorkowski, N. Sarafijanovic-Djukic, and M. Grossglauser. "CRAWDAD dataset epfl/mobility (v. 2009-02-24)." In: (Feb. 2009). DOI: 10.15783 / C7J010 (cited on pp. 68, 75).

[125] B. Poonguzharselvi and V. Vetriselvi. "Survey on routing algorithms in opportunistic networks." In: 2013 International Conference on Computer Communication and Informatics (2013), pp. 1-5 (cited on p. 17).

[126] Probabilistic Routing Protocol for Intermittently Connected Networks. https: //tools.ietf.org/html/rfc6693. Accessed: 12-12-2016 (cited on p. 22).

[127] J. M. Pujol, A. L. Toledo, and P. Rodriguez. "Fair routing in delay tolerant networks." In: Proceedings - IEEE INFOCOM (2009), pp. 837-845 (cited on p. 26). 
[128] J. Puttonen, S. Rantanen, F. Laakso, J. Kurjenniemi, K. Aho, and G. Acar. "Satellite model for network simulator 3." In: Proceedings of the 7th International ICST Conference on Simulation Tools and Techniques. ICST (Institute for Computer Sciences, Social-Informatics and Telecommunications Engineering). 2014, pp. 86-91 (cited on p. 24).

[129] R. Ramanathan, R. Hansen, P. Basu, R. Rosales-Hain, and R. Krishnan. "Prioritized epidemic routing for opportunistic networks." In: Proceedings of the 1st international MobiSys workshop on Mobile opportunistic networking - MobiOpp '07 (2007), pp. 62-66 (cited on pp. 18, 84).

[130] F. D. Rango, S. Amelio, and P. Fazio. "Epidemic Strategies in Delay Tolerant Networks from an Energetic Point of View : Main Issues and Performance Evaluation." In: Journal of Networks (2012) (cited on p. 85).

[131] U. Raza, P. Kulkarni, and M. Sooriyabandara. "Low Power Wide Area Networks: A Survey." In: (2016), pp. 1-15 (cited on p. 14).

[132] RFC-4838: Delay-Tolerant Networking Architecture. https://tools.ietf . org/html/rfc4838. Accessed: 20-11-2016 (cited on p. 21).

[133] RFC-5050: Bundle Protocol Specification. https : / / tools . ietf . org / html/rfc5050. Accessed: 20-11-2016 (cited on p. 21).

[134] I. Rhee, M. Shin, S. Hong, K. Lee, S. J. Kim, and S. Chong. "On the Levywalk Nature of Human Mobility." In: IEEE/ACM Trans. Netw. 19.3 (June 2011), pp. 630-643. ISSN: 1063-6692 (cited on p. 13).

[135] F. Richter. "An Average WhatsApp User Sends Messages per Month." In: (2015) (cited on pp. 44, 48, 58, 115).

[136] N. Ristanovic, G. Theodorakopoulos, and J. Y. L. Boudec. "Traps and pitfalls of using contact traces in performance studies of opportunistic networks." In: 2012 Proceedings IEEE INFOCOM. 2012, pp. 1377-1385 (cited on p. 8).

[137] J. A. Sanguesa, M. Fogue, P. Garrido, F. J. Martinez, J. C. Cano, and C. T. Calafate. "A Survey and Comparative Study of Broadcast Warning Message Dissemination Schemes for VANETs." In: Mobile Information Systems 2016 (2016) (cited on p. 69).

[138] SCAMPI Project. http://www.ict-scampi.eu (cited on p. 34). 
[139] M. R. Schurgot, C. Comaniciu, and K. Jaffres-Runser. "Beyond traditional DTN routing: social networks for opportunistic communication." In: IEEE Communications Magazine 50.7 (2012), pp. 155-162. ISSN: 0163-6804. DOI: 10.1109/MCOM.2012.6231292 (cited on p. 6).

[140] Sigfox. http://www.sigfox.com/. Accessed: 2017-02-20 (cited on p. 14).

[141] F. A. Silva, C. Celes, A. Boukerche, L. B. Ruiz, and A. A. Loureiro. "Filling the Gaps of Vehicular Mobility Traces." In: Proceedings of the 18th ACM International Conference on Modeling, Analysis and Simulation of Wireless and Mobile Systems. MSWiM '15 (2015), pp. 47-54 (cited on p. 48).

[142] O. Simeone and U. Spagnolini. "Capacity of wireless ad hoc networks with opportunistic collaborative communications." In: Eurasip Journal on Wireless Communications and Networking 2007 (2007) (cited on p. 100).

[143] A. Socievole, F. D. Rango, and C. Coscarella. "Routing approaches and performance evaluation in delay tolerant networks." In: 2011 Wireless Telecommunications Symposium (WTS) (2011), pp. 1-6 (cited on p. 6).

[144] T. Spyropoulos, K. Psounis, and C. Raghavendra. "Efficient Routing in Intermittently Connected Mobile Networks: The Multiple-Copy Case." In: Networking, IEEE/ACM Transactions on 16.1 (2008), pp. 77 -90 (cited on pp. 8, 22).

[145] T. Spyropoulos, K. Psounis, and C. S. Raghavendra. "Spray and Wait: An Efficient Routing Scheme for Intermittently Connected Mobile Networks." In: Proceedings of the 2005 ACM SIGCOMM Workshop on Delay-tolerant Networking (2005), pp. 252-259 (cited on pp. 6, 18, 22).

[146] T. Spyropoulos, K. Psounis, and C. S. Raghavendra. "Spray and focus: Efficient mobility-assisted routing for heterogeneous and correlated mobility." In: Proceedings - Fifth Annual IEEE International Conference on Pervasive Computing and Communications Workshops, PerCom Workshops 2007 (2007), pp. 79-85 (cited on p. 26).

[147] T. Spyropoulos, T. Turletti, and K. Obraczka. "Routing in delay-tolerant networks comprising heterogeneous node populations." In: IEEE Transactions on Mobile Computing 8.8 (2009), pp. 1032-1047 (cited on p. 26).

[148] J. Su, A. Chin, A. Popivanova, A. Goel, and E. D. Lara. "User Mobility for Opportunistic Ad-Hoc Networking." In: Sixth IEEE Workshop on Mobile 
Computing Systems and Applications (WMCSA 2004) (Low Wood, United Kingdom, 2004) (cited on p. 85).

[149] Q. Technologies Incorporated. "WHEN MOBILE APPS USE TOO MUCH POWER A Developer Guide for Android App Performances." In: Technologies Incorporated, Qualcomm (2013) (cited on p. 32).

[150] G. S. Thakur, U. Kumar, A. Helmy, and W.-J. Hsu. "On the efficacy of mobility modeling for DTN evaluation: Analysis of encounter statistics and spatio-temporal preferences." In: Wireless Communications and Mobile Computing Conference (IWCMC), 2011 7th International (Istanbul, Turkey, 2011), pp. 510-515 (cited on p. 5).

[151] K. Thilakarathna, A. C. Viana, A. Seneviratne, and H. Petander. "Mobile social networking through friend-to-friend opportunistic content dissemination." In: Proceedings of the fourteenth ACM international symposium on Mobile ad hoc networking and computing - MobiHoc '13 (2013), p. 263 (cited on p. 100).

[152] S. Tornell, C. Calafate, J.-C. Cano, and P. Manzoni. "DTN Protocols for Vehicular Networks: an Application Oriented Overview." In: IEEE Communications Surveys \& Tutorials (2015), pp. 868-887 (cited on pp. 6, 17, $69)$.

[153] S. M. Tornell, S. Patra, C. T. Calafate, J. C. Cano, and P. Manzoni. "GRCBox: Extending smartphone connectivity in vehicular networks." In: International Journal of Distributed Sensor Networks 2015 (2015) (cited on p. 58).

[154] T.-C. Tsai and H.-H. Chan. "NCCU Trace: social-network-aware mobility trace." In: Communications Magazine, IEEE 53 (2015), pp. 144-149 (cited on pp. 12, 44, 103, 114).

[155] University of Dartmouth. CRAWDAD: A Community Resource for Archiving Wireless Data (cited on pp. 8, 12).

[156] Use AirDrop to send content from your Mac. https://support . apple. com/en-nz/HT204144/. Accessed: 19/10/2015 (cited on p. 86).

[157] A. Vahdat and D. Becker. "Epidemic routing for partially connected ad hoc networks." In: Technical report number CS-200006, Duke University (2000), pp. 1-14 (cited on pp. 6, 18, 22, 58). 
[158] D. Vardalis and V. Tsaoussidis. "Exploiting the potential of DTN for energy-efficient internetworking." In: Journal of Systems and Software 90 (2014), pp. 91-103 (cited on p. 85).

[159] A. M. Vegni, C. Campolo, A. Molinaro, and T. D. C. Little. "Modeling of Intermittent Connectivity in Opportunistic Networks: The Case of Vehicular Ad hoc Networks." In: Routing in Opportunistic Networks (2013) (cited on p. 58).

[160] V. Vukadinovic and S. Mangold. "Opportunistic Wireless Communication in Theme Parks: A Study of Visitors Mobility." In: Proceedings of the 6th ACM Workshop on Challenged Networks. CHANTS '11. New York, NY, USA: ACM, 2011, pp. 3-8 (cited on p. 8).

[161] F. Warthman. "Delay-and Disruption-Tolerant Networks (DTNs) A Tutorial, version 2.0." In: The InterPlaNetary (IPN) Internet Project. InterPlanetary Networking Special Interest Group (IPNSIG) (2012) (cited on p. 5).

[162] K. Wehrle, M. Günes, and J. Gross. Modeling and Tools for Network Simulation. Springer, 2010 (cited on p. 16).

[163] K. Wei, S. Guo, and K. Xu. "CACC: A context-aware congestion control approach in smartphone networks." In: IEEE Communications Magazine 52.6 (2014), pp. 42-48 (cited on p. 26).

[164] J. Whitbeck, V. Conan, and M. Dias de Amorim. "Performance of Opportunistic Epidemic Routing on Edge-Markovian Dynamic Graphs." In: Communications, IEEE Transactions on 59.5 (2011), pp. 1259-1263 (cited on p. 8).

[165] J. Whitbeck, V. Conan, and M. D. de Amorim. "Performance of Opportunistic Epidemic Routing on Edge-Markovian Dynamic Graphs." In: IEEE Transactions on Communications 59 (2011), pp. 1259-1263 (cited on p. 84).

[166] F. Xia, L. Liu, J. Li, J. Ma, and A. V. Vasilakos. "Socially Aware Networking: A Survey." In: IEEE Systems Journal 9.3 (2015), pp. 904-921. ISSN: 1932-8184. DOI: 10.1109/JSYST.2013.2281262 (cited on p. 6).

[167] M. Xu. "Collaborative opportunistic network coding for persistent data stream in disruptive sensor networks." In: 2013 IEEE International Con- 
ference on Pervasive Computing and Communications Workshops, PerCom Workshops 2013 March (2013), pp. 413-414 (cited on p. 100).

[168] Q. Xu, Z. Su, K. Zhang, P. Ren, and X. Shen. "Epidemic Information Dissemination in Mobile Social Networks with Opportunistic Links." In: IEEE Transactions on Emerging Topics in Computing 6750 (2015), pp. 11 (cited on p. 84).

[169] Q. Xu, Z. Su, K. Zhang, P. Ren, and X. S. Shen. "Epidemic Information Dissemination in Mobile Social Networks With Opportunistic Links." In: Emerging Topics in Computing, IEEE Transactions on 3.3 (2015), pp. 399409 (cited on p. 8).

[170] S. Yang, X. Yang, C. Zhang, and E. Spyrou. "Using social network theory for modeling human mobility." In: IEEE network 24.5 (2010) (cited on p. 13).

[171] R. Zhang, A. R. Chandran, N. Timmons, and J. Morrison. "OppSim: A Simulation Framework for Opportunistic Networks Based on MiXiM." In: Proceedings of the IEEE 19th International Workshop on Computer Aided Modeling and Design of Communication Links and Networks, CAMAD 2014. 2014 (cited on p. 23).

[172] X. Zhang, G. Neglia, J. Kurose, and D. Towsley. "Performance modeling of epidemic routing." In: Computer Networks 51.10 (2007), pp. $2867-2891$ (cited on p. 8).

[173] Y. Zhang and J. Zhao. "Social network analysis on data diffusion in delay tolerant networks." In: Proceedings of the tenth ACM international symposium on Mobile ad hoc networking and computing - MobiHoc '09 (2009), pp. 345-346 (cited on p. 84).

[174] Z. Zhao, B. Mosler, and T. Braun. "Performance evaluation of opportunistic routing protocols: a framework-based approach using OMNeT++." In: Proceedings of the 7th Latin American Networking Conference. ACM. 2012, pp. 28-35 (cited on p. 23).

[175] D. Zhu and M. Mutka. "Promoting cooperation among strangers to access Internet services from an ad hoc network." In: Pervasive and Mobile Computing 1.2 (2005), pp. 213-236 (cited on p. 100). 
[176] H. Zhu, L. Fu, G. Xue, Y. Zhu, M. Li, and L. M. Ni. "Recognizing exponential inter-contact time in VANETs." In: Proceedings of the 29th conference on Information communications. INFOCOM'10. Piscataway, NJ, USA: IEEE Press, 2010, pp. 101-105 (cited on p. 12).

[177] H. Zhu and M. Li. "Dealing with Vehicular Traces." In: Studies on Urban Vehicular Ad-hoc Networks (2013), pp. 15-21 (cited on pp. 44, 69). 